\title{
EFFICIENT VIDEO TRANSMISSION OVER CORRELATED NAKAGAMI FADING CHANNELS \\ FOR IS-95 CDMA SYSTEMS
}

by

NORMAN CHAN

B. Eng. (Electrical Engineering), McMaster University, Canada, 1994

A THESIS SUBMITTED IN PARTIAL FULFILLMENT OF

THE REQUIREMENTS FOR THE DEGREE OF

MASTER OF APPLIED SCIENCE

in

THE FACULTY OF GRADUATE STUDIES

DEPARTMENT OF ELECTRICAL ENGINEERING

We accept this thesis as conforming

to the required standard

THE UNIVERSITY OF BRITISH COLUMBIA

April 1999

() Norman Chan, 1999 
In presenting this thesis in partial fulfilment of the requirements for an advanced degree at the University of British Columbia, I agree that the Library shall make it freely available for reference and study. I further agree that permission for extensive copying of this thesis for scholarly purposes may be granted by the head of my department or by his or her representatives. It is understood that copying or publication of this thesis for financial gain shall not be allowed without my written permission.

Department of ELECTRICAL ENGINEERING

The University of British Columbia Vancouver, Canada

Date April 20,1999 


\begin{abstract}
\end{abstract}
This thesis deals with the problem of efficient transmission of video signals over generalized fading channels in Direct Sequence-Spread Spectrum (DS-SS) Code Division Multiple Access (CDMA) systems. The video codec is based upon the ITU H.263 compression algorithm which targets at providing low bit-rate video telephony services suitable for wireless transmission. In order to reduce the overall impact of errors due to mobile channels on the video sequence, a modified version of the original H.263 codec is proposed incorporating a selective Forward Error Correction (FEC) coding scheme combined with a forced INTRA frame update mechanism. This modified version of H.263 codec provides improvement in both average video and frame-to-frame performance. We further consider a coherent DS-CDMA system based upon the IS-95 standard for the forward link (base-to-mobile) in both single-cell and multiple-cell environment. We provide performance evaluation results by both analysis, employing the Gaussian approximation, and computer simulations, using Monte Carlo error counting techniques. The proposed CDMA system uses concatenated Walsh/maximal-length coding scheme for spectrum spreading. The resulting spread codes maintain orthogonality while reducing inhomogenous cross-correlations among Walsh sequences. The frequency-selective fading channel is modeled by a tapped delay line model with channel coefficients of each path following an independent Nakagami- $m$ distribution. We have implemented in software a correlated Nakagami fading simulator based upon the principle of superposition of complex partial waves with a (possibly strong) component resulting from the direct waves. The time correlation is generated by assigning each partial wave a Doppler shift as a function of time. This approach is an exact replica of the actual physical situation as it reproduces the wave propagation process, as opposed to the Doppler spectrum approximation approach used in other simulators. The received signal is demodulated coherently using a RAKE 
receiver with variable resolving fingers, where multipath components are maximal-ratio combined. For our analysis, we assume perfect knowledge of the channel, which could be accomplished either by the usage of pilot tone or some type of channel-parameter estimation circuits. However, for the computer simulation, such perfect knowledge of the channel is not necessary.

In terms of performance evaluation results, we first present the improved performance of the modified H.263 codec as a function of Peak Signal-to-Noise Ratio (PSNR) transmitted in additive white Gaussian noise (AWGN) environment. Then, the analytical and computer simulated results for the bit error rate (BER) performance of CDMA forward link in Nakagami fading channels for both single-cell and multiple-cell environment are presented. Further, we present the PSNR performance results for the video transmission featuring the modified H.263 coding scheme over the proposed CDMA systems. Finally, a variety of performance evaluation results, both in single-cell and multiple-cell environment, are presented for different number of resolving paths, signal propagation characteristics, cell user capacity, as well as for the presence of channel estimation errors. In all cases, heuristic explanations and interpretations of the trend of the obtained results are also given. 


\section{Table of Contents}

Abstract

List of Figures

Chapter 1 INTRODUCTION 1

$\begin{array}{lll}\text { Chapter } 2 \text { BACKGROUND } & 5\end{array}$

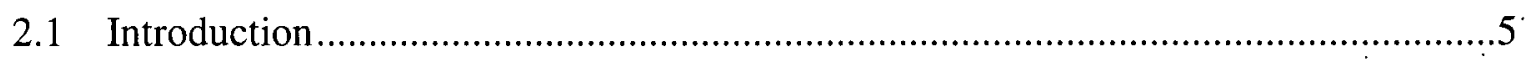

2.2 ITU-T H.263 Low Bit-Rate Video Compression ..............................................5

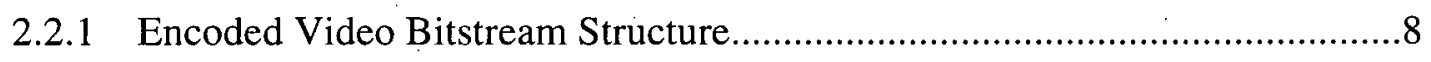

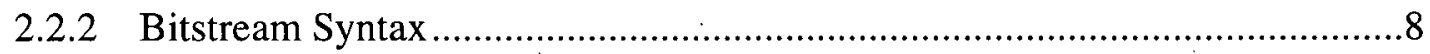

2.3 Mobile Radio Propagation: Large-Scale Fading ..............................................11

2.4 Mobile Radio Propagation: Small-Scale Fading ............................................12

2.4.1 Small-Scale Fading: Parameters and Characterization ..............................12

2:4.2 Small-Scale Fading: Distribution Models.........................................14

2.5 Spread Spectrum Modulation Techniques .................................................... 17

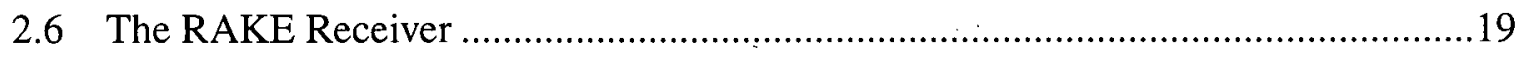

2.7 IS-95 Code Division Multiple Access System............................................20

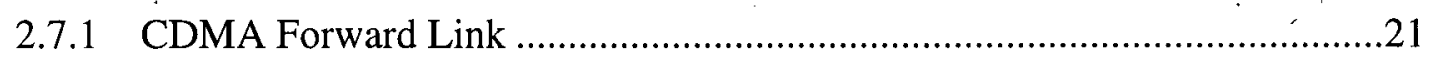

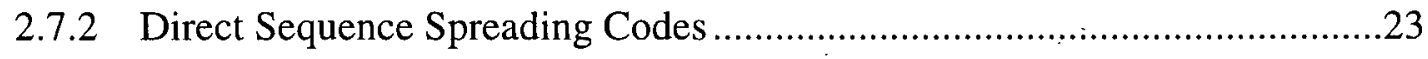

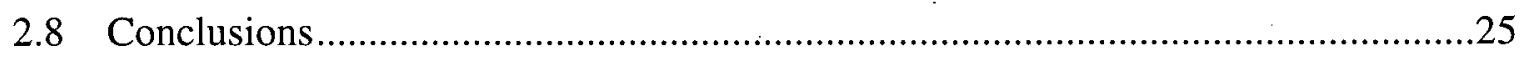

Chapter 3 MODIFIED H.263 VIDEO CODEC $\cdots 27$

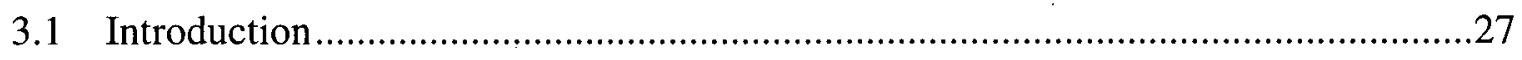

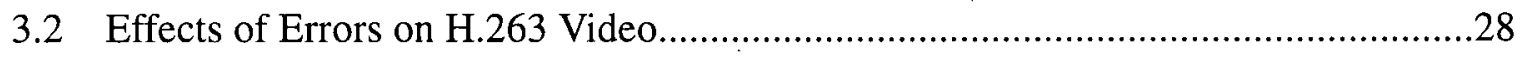

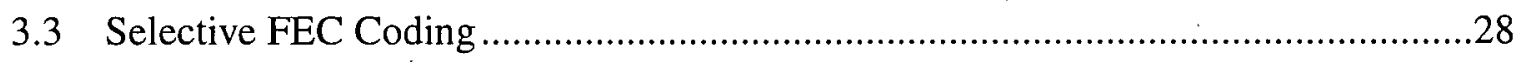




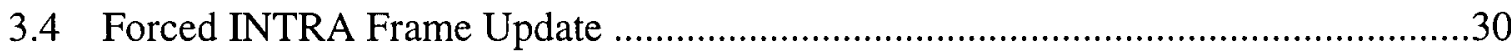

3.5 Numerical Results for the AWGN Channel ...........................................................

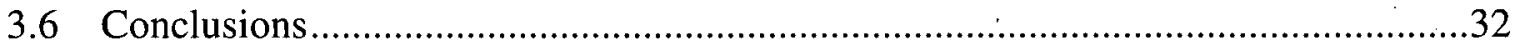

Chapter 4 FORWARD LINK VIDEO TRANSMISSION IN SINGLE-CELL CDMA SYSTEMS

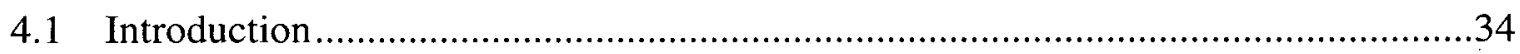

4.2 Direct Sequence-Spread Spectrum (DS-SS) CDMA System Model.........................36

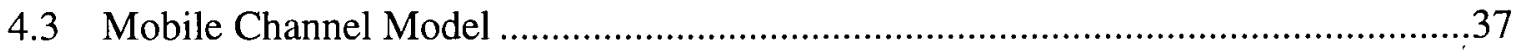

4.4 Correlated Nakagami Fading Simulator ...............................................................39

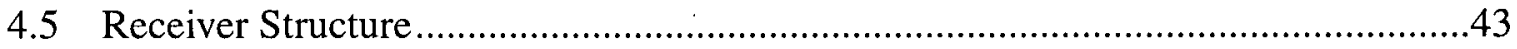

4.6 CDMA Forward Link BER Performance Analysis ...................................................44

4.7 Forward Link BER Analysis with Gaussian Approximation ...................................48

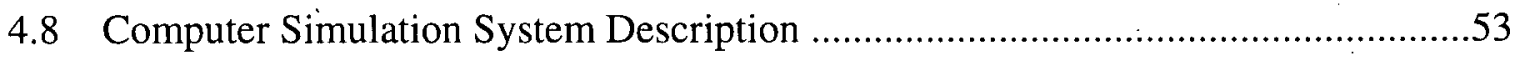

4.9 BER Performance Evaluation Results for CDMA Forward Link ..............................54

4.10 Video Transmission Performance in CDMA Forward Link ....................................58

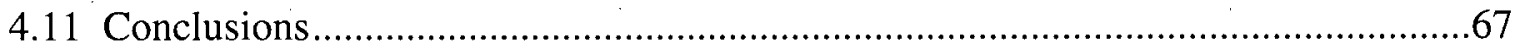

\section{Chapter 5 FORWARD LINK VIDEO TRANSMISSION IN MULTIPLE-CELL CDMA SYSTEMS $\quad 69$}

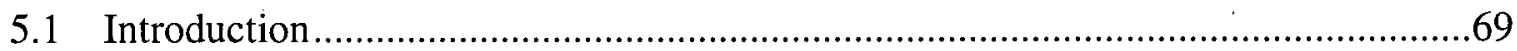

5.2 Multiple-Cell Configuration Model .....................................................................70

5.3 Channel Modeling ..............................................................................................71

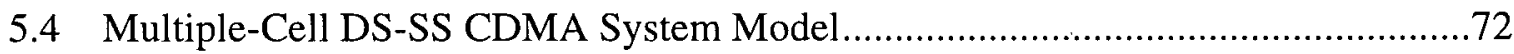

5.5 Multiple-Cell CDMA Forward Link BER Performance Analysis ............................73

5.6 Multiple-Cell BER Performance Analysis with Gaussian Approximation ................74 
5.7 Computer Simulation Model Description ....................................................80

5.8 BER Performance Evaluation Results for Multiple-Cell Systems ........................80

5.9 Video Transmission Performance in Multiple-Cell Systems ..............................82

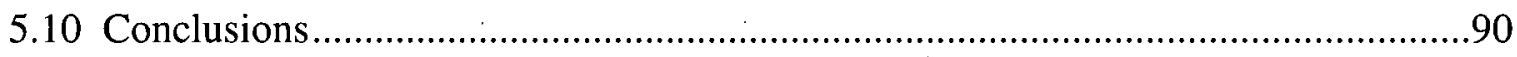

Chapter 6 CONCLUSIONS AND FUTURE RESEARCH $\quad \cdot 92$

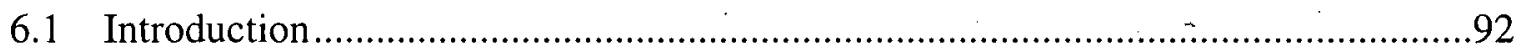

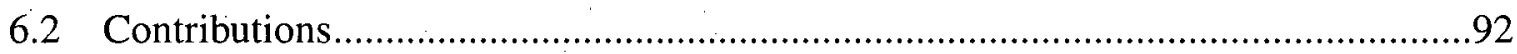

6.2.1 A Modified H.263 Video Codec ..........................................................92

6.2.2 Correlated Nakagami Fading Simulator ......................................... 92

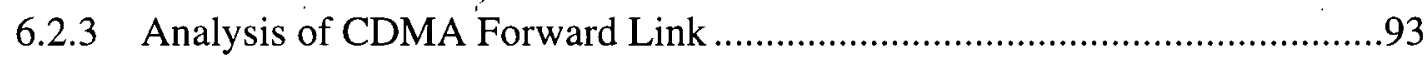

6.2.4 End-to-end Video Transmission Performance Evaluation .........................93

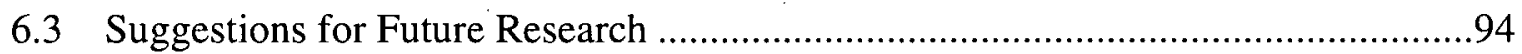

Glossary $\quad 95$

$\begin{array}{ll}\text { Bibliography } \cdot & 101\end{array}$

Appendix A. Derivations of Multiple-Access Interference Variance for Gaussian $\begin{array}{ll}\text { Approximation } & 106\end{array}$

$\begin{array}{ll}\text { Appendix B. Evaluation of the Mean of Attenuation Factor } & 109\end{array}$ 


\section{List of Figures}

Fig. $2.1 \quad$ H.263 Video Encoder Block Diagram...........................................................6

Fig. 2.2 Picture Format and Resolutions for QCIF in H.263...........................................

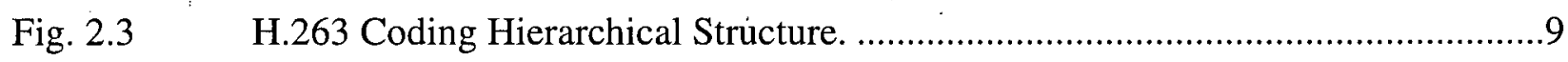

Fig. 2.4 Simplified H.263 Video Bitstream Syntax......................................................10

Fig. $2.5 \quad$ Signal Spectra Before and After Spreading........................................................

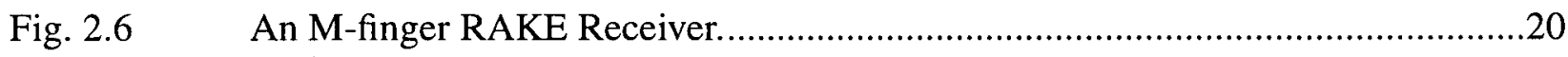

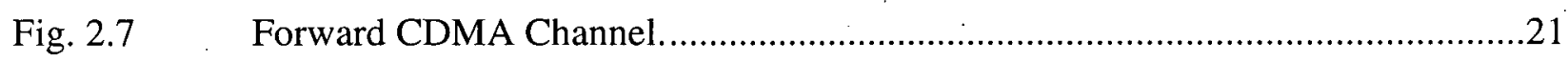

Fig. 3.1 Sample Frames of Miss America Video Sequence (Frame \#0 and 90) ..................31

Fig. 3.2 Video Performance of Modified H.263 Codec ………............................................32

Fig. 3.3 Frame Performance of the Modified H.263 Codec ...............................................33

Fig. 4.1 Simulated DS-SS CDMA Forward Link Video Transceiver System Model ..........35

Fig. 4.2 Simulated DS-SS CDMA System Model …………….......................................

Fig. 4.3 Tapped Delay Line Model of Frequency-Selective Fading Channel. .....................39

Fig. 4.4 Example of a Phasor Summation of Partial Waves..............................................41

Fig. 4.5 Comparison of Nakagami Fading Simulator and PDF for $m=1$ …....................43

Fig. 4.6 Comparison of Nakagami Fading Simulator and PDF at for $m=3 \ldots \ldots \ldots \ldots \ldots \ldots \ldots . . .43$

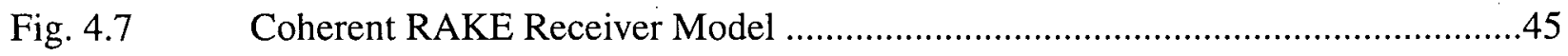

Fig. $4.8 \quad$ Single-Cell CDMA Forward Link BER Performance …………….......................56

Fig. 4.9 Simulation Results Comparison of Concatenated and Non-Concatenated

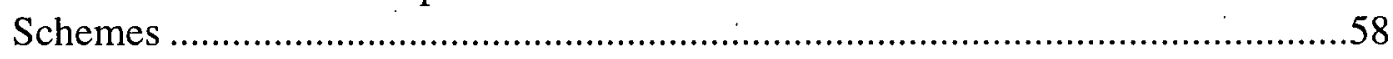

Fig. 4.10 Video Performance of Modified H.263 Codec in Single-Cell CDMA System .....61

Fig. 4.11 PSNR Performance with Various Number of RAKE Fingers ...............................62 
Fig. 4.12 PSNR Performance with Different Fading Figures 63

Fig. 4.13 PSNR Performance with Different Logarithmic MIP Exponents...........................64

Fig. 4.14 PSNR Performance with Different Mobile Velocities ...........................................66

Fig. 4.15 PSNR Performance with Non-Identical Multipath Fading for Each Path .............67

Fig. 4.16 PSNR Performance with Imperfect Channel Estimation.......................................68

Fig. $5.1 \quad$ Multiple-Cell Configuration Model ……….......................................................

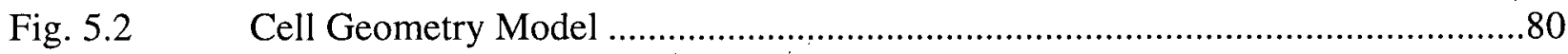

Fig. 5.3 Analysis vs. Simulation for Multi-cell System ……..............................................

Fig. 5.4 PSNR Performance with Non-Identical $m$ Values for Each Multipath...................86

Fig. 5.5 PSNR Performance with Non-Identical $m$ Value Set for Channels from .

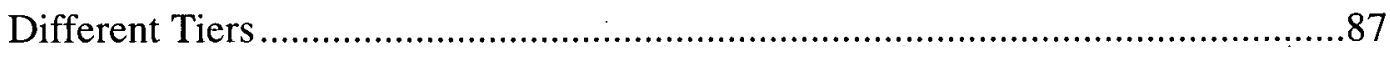

Fig. 5.6 PSNR Performance with Different Surrounding-Cell User Load...........................89

Fig. 5.7 PSNR Performance with Non-Identical MIP for Channels from Different Tiers..90

Fig. 5.8 PSNR Performance with Imperfect Channel Estimation .....................................91

Fig. 5.9 PSNR Performance with Non-Identical Number of Multipaths for Different Tiers 


\section{Acknowledgments}

This thesis is dedicated to my family, whom I am forever indebted to, for their love and support. From the bottom of my heart, I thank my supervisor, Dr. P. Takis Mathiopoulos, who has always been so supportive and helpful, for his guidance, encouragement, and financial support throughout my thesis work. I really appreciate his friendly personality and his confidence in me, which make everything so much easier during the course of work. I would also like to thank everyone in the Communications Group for their help and friendship, as they have tremendously enriched my campus life at UBC. Finally, I would like to express my gratitude to the financial support provided by the University Graduate Fellowship and the NSERC scholarship. 


\section{Chapter 1 INTRODUCTION}

In recent years, Code Division Multiple Access (CDMA) systems have become a viable alternative to both Frequency Division Multiple Access (FDMA) and Time Division Multiple Access (TDMA) schemes for mobile cellular telecommunication systems. The use of spread spectrum techniques in wireless communications applications has been a very active area topic for research and development [1], [2], [3]. The CDMA "Mobile Station-Base Station Compatibility Standard for Dual Mode Wideband Spread Spectrum Cellular System," was issued as IS-95 in December 1992 [9]. It provides a common framework for wireless service providers to develop new compatible CDMA networks. Since its release, CDMA has become a popular choice for new cellular systems around the world. Direct Sequence-Spread Spectrum (DS-SS) technique used in the IS95 systems allows sharing of the same frequency band for all users within each cell and among multiple cells, thus greatly simplifies frequency planning within a market. In addition, the inherent wide bandwidth nature of spread spectrum waveforms provides effective fading mitigation and interference combating ability. Due to these distinct advantages of spread spectrum, and the fact that majority of the third generation cellular system proposal adopt spread spectrum techniques [4], this technology seems to be a very promising area worth investigating.

As wireless communications become more popular, information exchange is no longer confined to basic voice transmission. In the last few years, the demand for wireless multimedia services such as the transmission of text data, voice, still images, and video has grown rapidly [10]. Among those services, wireless digital video transmission has probably the most profound impact on the development of future wireless telecommunication applications [11]. At the same time, it also brings along with it many challenges for systems design. To transmit video through 
limited bandwidth of radio channels, the first problem is to compress the huge amount of video data to a manageable size that the wireless networks can handle. Among many video compression schemes, ITU-T H.263 low bit-rate video coding standard [12], which targets for videophone and videoconferencing applications, provides a very promising solution. Its low-bit coding scheme aims at compressing video data at rates of no more than $64 \mathrm{kbit} / \mathrm{s}$, low enough for transmission through Public Switched Telephone Networks (PSTN) as well as wireless networks. However, there exists a trade-off between compression performance and error sensitivity. The higher the compression rate of the video encoder, the more sensitive channel errors affect the decoded video. Given the possibly severe error-prone nature of the wireless environment, special error control scheme has to be incorporated in the video codec for satisfactory performance in unreliable mobile channels.

In [13]-[21] and [49], there have been a variety of approaches investigated for video transmission over wireless networks. However, only a few of them considered an end-to-end multiple access radio link [13], [18], [19], [20], [21]. Among them, only [13] and [49] uses CDMA channels for video transmission simulations. Most of these papers have dealt with single cell environment but have not taken into account the effects of multiple-cell interference. Also, the mobile channel models they employed usually assume Rayleigh fading characteristics; however, in the problem of obtaining the distribution of the signal strength for each fading multipath, Nakagami- $m$ distribution gives a more general solution [24] compared to Rayleigh and Rician distributions which provide only special case solutions. Empirical data from [22] also suggests that path fading statistics are more adequately described by Nakagami- $m$ fading. In general, Nakagami distribution provides a more general and versatile way to model wireless channels since it can model a greater and wider variety of fading environments. 
Motivated by the above, in this thesis, we are addressing the problem of efficient transmission of video data through IS-95 based CDMA systems over correlated Nakagami fading channels. In particular, the contributions of this thesis can be summarized as follows:

- A modified version of the H.263 video codec is proposed for improved transmission performance in error-prone mobile environment.

- A correlated Nakagami fading simulator is implemented in software for more general and versatile' fading channel modeling.

- BER analysis for the DS-SS CDMA forward link in both single-cell and multiple-cell environment are performed. Numerical results are evaluated and compared with those obtained from the Monte Carlo simulations.

- End-to-end video transmission through a IS-95 based CDMA system over correlated Nakagami fading channels is simulated. Transmission performance results of the modified H.263-encoded video data in both single-cell and multiple-cell environment are evaluated under a wide variety of system conditions.

The following is the organization of this thesis. In Chapter 2, background material for this thesis is outlined. ITU-T H.263 video coding scheme is summarized and its coding syntax related to the thesis is elaborated. Then, mobile channel characteristics including large-scale fading and different kinds of small-scale fading are described with emphasis in Nakagami- $m$ fading and its relationships with conventional Rayleigh and Rician fading models. Next, the basic concept of spread spectrum and its inherent advantages in CDMA systems are described. Lastly, the IS-95 CDMA forward link and its major components are presented.

In Chapter 3, introduction of the proposed modified H.263 video codec is presented. This chapter includes a detailed description of the structure of the proposed codec as well as various 
evaluation results under additive white Gaussian noise (AWGN) channel condition.

In Chapter 4, a DS-SS CDMA system operating in a single-cell environment is investigated. We first present both analytical and computer simulated CDMA systems, including the channel model used for the Nakagami fading. Then, the analysis of the bit error rate (BER) performance of the forward link is presented. Numerical results of the BER performance evaluated from both the analysis and the computer simulations are compared. Lastly, we present and discuss the simulation results of the end-to-end video transmission performance utilizing the proposed H.263 codec over a IS-95 based CDMA forward link system.

In Chapter 5, the forward link of a DS-SS CDMA system operating in a multiple-cell environment is investigated. We describe the models for the cellular configuration, the multiplecell radio channels as well as the CDMA forward link system. Then, the BER performance analysis is presented and its numerical results are compared with those obtained from Monte Carlo simulations. Numerical results of the video transmission performance over the multiple-cell CDMA forward link in correlated Nakagami fading channels are evaluated through computer simulations.

Finally, in Chapter 6, we present our conclusions and suggest potential topics for future studies. 


\section{Chapter 2 BACKGROUND}

\subsection{Introduction}

The purpose of this chapter is to provide for the background knowledge of the compression scheme, channel modeling, spread spectrum systems and standard relevant to this thesis. In Section 2.2, we describe the ITU H.263 low bit-rate video compression scheme by covering its encoding mechanism, video format, hierarchical structure, and bitstream syntax. It is followed by the discussion of mobile radio propagation channels in Section 2.3 and 2.4, where large-scale and small-scale fading models are presented. In Section 2.5, an overview of spread spectrum modulation techniques is provided. We then explain the diversity mechanism of RAKE receiver in Section 2.6. We conclude the chapter by describing the IS-95 CDMA system for the forward link and discussing the characteristics of the spreading codes the standard employs.

\subsection{ITU-T H.263 Low Bit-Rate Video Compression}

In the past few years, with the emerge of multimedia services, there has been great demand for digital video communications. Thus, the ITU-T (formerly CCITT) H.263 standard [12] for low bit-rate video compression scheme was proposed for videophone and videoconferencing applications. The targeted bit-rate, for less than $64 \mathrm{kbit} / \mathrm{s}$, is relatively low compared to other standardized video compression schemes such as MPEG-2 [54], of which bit-rates are in the range of $\mathrm{Mbits} / \mathrm{s}$. It is due to this low bit-rate compression that makes H.263 video encoding scheme suitable for wireless mobile applications [21], in which bandwidth constraint imposes a major design problem. In order to achieve the high compression ratio, H.263 video coder incorporates a combined effort of two dimensional ( $8 \times 8$ ) Discrete Cosine Transform (DCT) and motion- 
compensation prediction to compress moving images in spatial and temporal domains, respectively.

The block diagram of the H.263 video encoder is shown in Fig. 2.1 [12]. The Coding

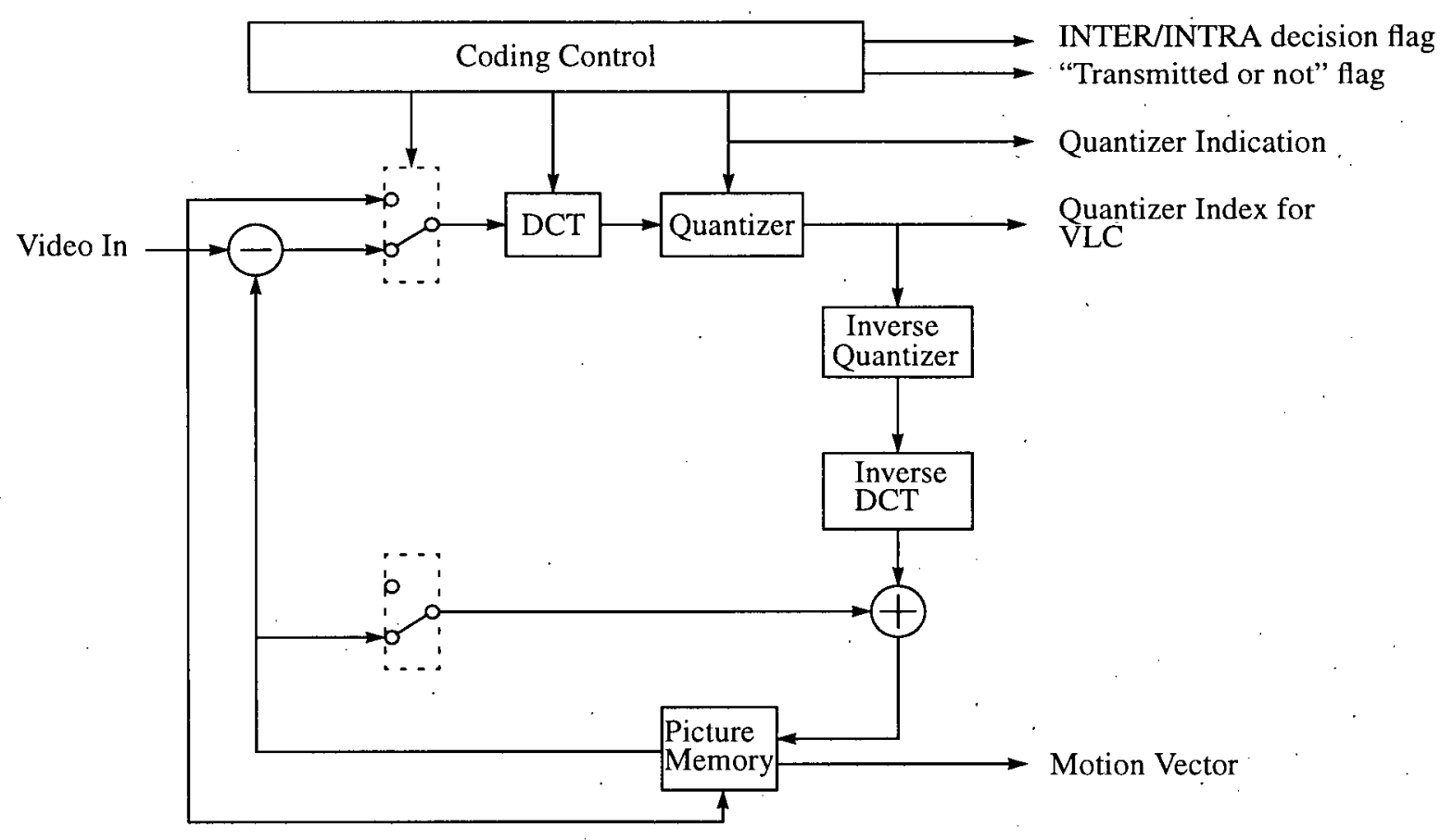

Fig. 2.1 H.263 Video Encoder Block Diagram [12].

Control module determines the INTER/ INTRA decision flag. It is used to indicate if the encoder is in inter or intra mode. The encoder is said to be in intra mode if it operates DCT directly on the input image without the use of motion compensation. The resulting frame is called an INTRA or I-frame. Otherwise, the encoder is said to be in inter mode and the resulting frame is called a Prediction or P-frame. Coding Control also determines the value of "Transmitted or not" flag. The flag is set to 1 if coded data is transmitted, or 0 if none is transmitted in which case data from previous frame is reused. The Quantizer Indication from Coding Control provides quantization step size information. 
Each video image is divided into blocks of $8 \times 8$ pixels before being sent to the encoder input as Video In [12]. When the encoder is in inter mode, prediction generated by the previous frame is subtracted from each block. The difference between the block being encoded and the prediction is sent for transform coding by the DCT block. On the other hand, when the encoder is in intra mode, the block is directly sent for DCT coding. In either case, the resulting transform coefficients are uniformly quantized by the Quantizer block. The resulting Quantization Index are then encoded using Variable Length Coding (VLC) such as Huffman codes. The quantized transform coefficients are also sent to Inverse Quantizer and Inverse DCT blocks. They are used to generate prediction frame which is stored in Picture Memory block for subsequent INTRA frame encoding and to generate the Motion Vector (MV) from motion-compensated prediction.

Fig. 2.2 shows the picture format and resolutions for Quarter Common Intermediate Format (QCIF) of video sequence used in the H.263 compression scheme [54]. QCIF is a univer-

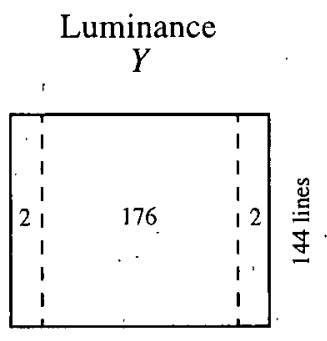

180 pixels
Chrominance

$\mathrm{Cb}$

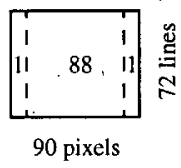

Chrominance

$\mathrm{Cr}$

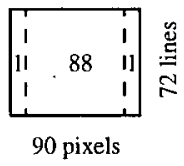

Fig. 2.2 Picture Format and Resolutions for QCIF in H.263 [54].

sal video format designed to accommodate different kinds of existing television formats such as PAL, SECAM and NTSC. It consists of one luminance component $Y$ and two chrominance components $C_{b}$ and $C_{r}$. Since the basic block size of $\mathrm{H} .263$ is $8 \times 8$ pixels, the pixels outside the dotted lines in Fig. 2.2 is cropped out, and the resulting area is called the Significant Pel Area 
(SPA). Thus, the $Y$ component has a resolution of 176 pixels $x 144$ lines, whereas $C_{b}$ and $C_{r}$ both have a resolution of 88 pixels $x 72$ lines. The $Y C_{b} C_{r}$ color coordinate adopted by $\mathrm{H} .263$ standard was developed as part of IYU-R BT.601 [25] during the establishment of a worldwide digital video component standard. This yields a compatible digital approach between the two different systems namely, the 525-line NTSC and 625-line PAL/ SECAM.

\subsubsection{Encoded Video Bitstream Structure}

The H.263 standard defines a consistent structure so that the decoder may decode the received bitstream without any ambiguity. The H.263 syntax for the coded video bitstream has a hierarchical representation (Fig. 2.3) with four data layers, namely the Picture Layer, the Group of Block (GOB) Layer, the Macro Block (MB) Layer and the Block ( 8 x 8 pixels) Layer [12]. Each layer is composed of data and the corresponding header information.

In Fig. 2.3, the Picture Layer divides a QCIF picture frame into 9 Group of Blocks denoted as GOB 0 to GOB 8 [12]. In the Group of Blocks Layer, each of the GOBs in a picture frame is sub-divided into rows of 11 Macroblocks. Each MB in turns consists of 1 luminance $(Y)$

component and 2 chrominance $\left(C_{b}\right.$ and $\left.C_{r}\right)$ components in the Macroblock Layer. The $Y$ component is made up of 4 basic blocks ( $8 \times 8$ pixels), where the $C_{b}$ and $C_{r}$ components are each made up of 1 basic block, i.e., half the spatial resolutions of luminance. Finally, the Block Layer consists of basic blocks which are $8 \times 8$ pixels in dimension.

\subsubsection{Bitstream Syntax}

Fig. 2.4 shows the simplified H.263 video bitstream syntax of each hierarchical layer of the coding [21]. It consists of the Picture Layer, the Group of Blocks Layer, the Macroblock Layer 


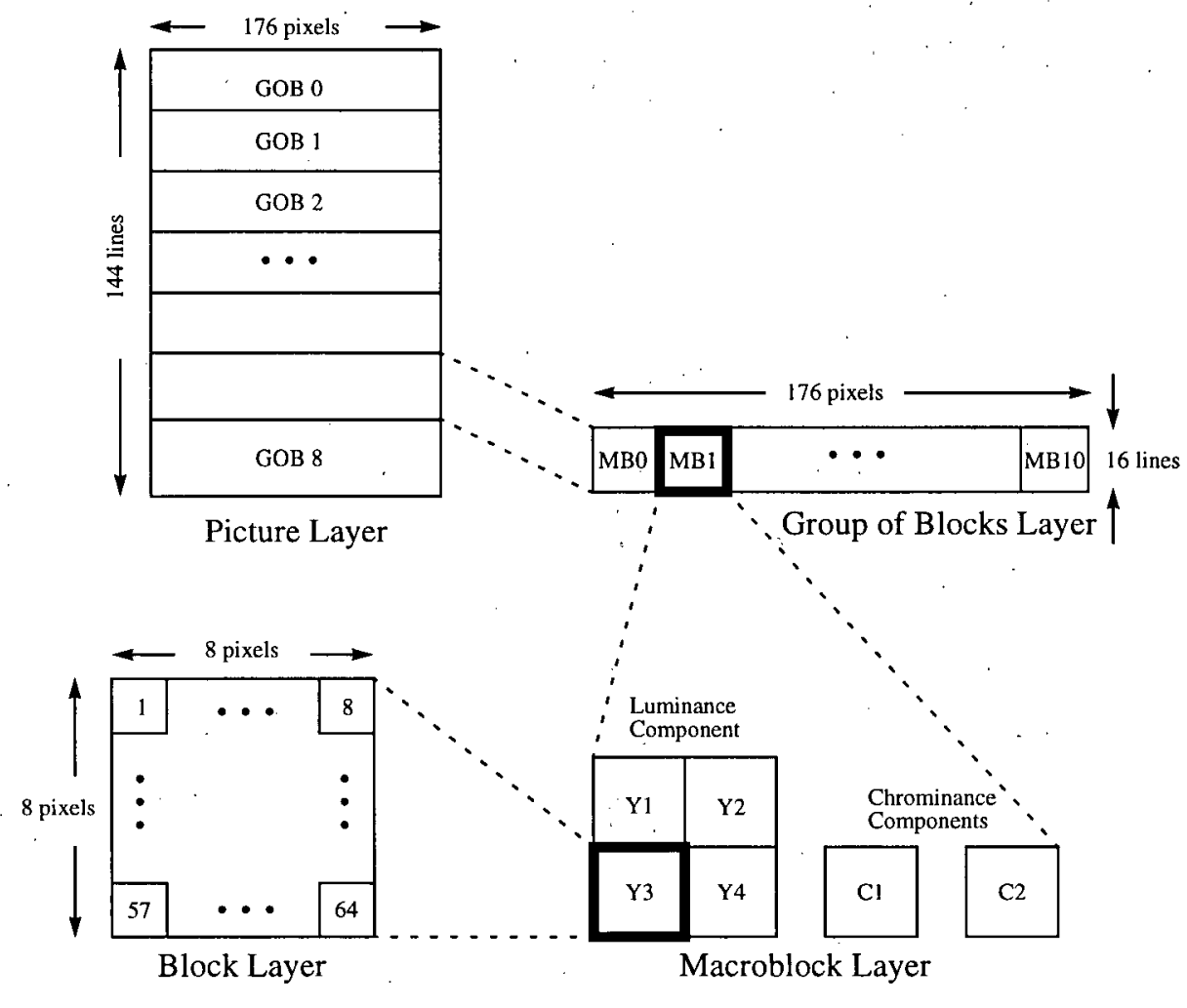

Fig. 2.3 H.263 Coding Hierarchical Structure [54].

and the Block Layer. The Picture Layer begins with the Picture Start Code (PSC), followed by the Picture Header and the data for the GOB layer [12]. It terminates with an optional End-OfSequence code (EOS) and stuffing bits (STUF). The Picture header consists of Temporal Reference (TR), Picture Type information (PTYPE), Picture Quantizer information (PQUANT), Continuous Presence Multipoint indicator (CPM), Picture Sub-Bitstream Indicator (PSBI), Extra Insertion information (PEI), and spare bits (SPARE). Temporal Reference for Bi-directional frames (TRB) and quantization information for bi-directional picture (DBQUANT) are also used in the Picture layer if optional PB-frames mode is used.

A GOB Layer consists of a GOB header followed by data for the Macroblock layer [12]. For the GOB that starts at the beginning of Macroblock row number 0 , the GOB header is not 
Picture Layer

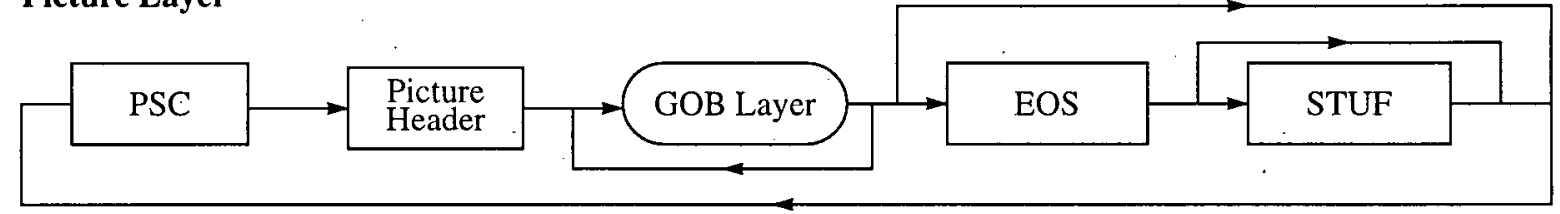

Group of Blocks (GOB) Layer

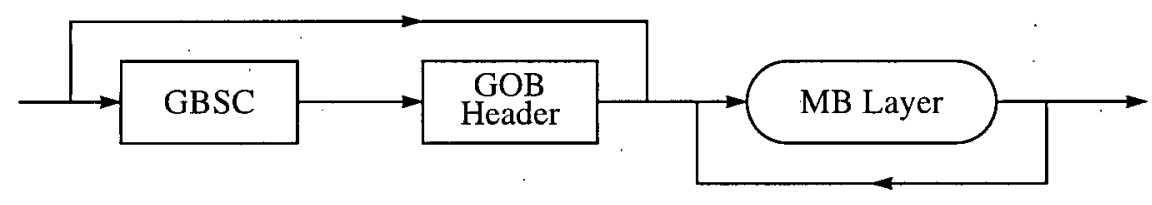

Macroblock (MB) Layer

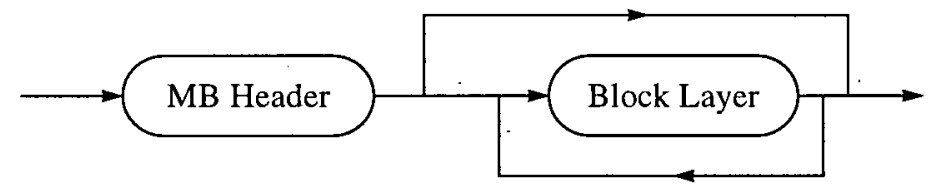

Fixed Length Coding

Block Layer

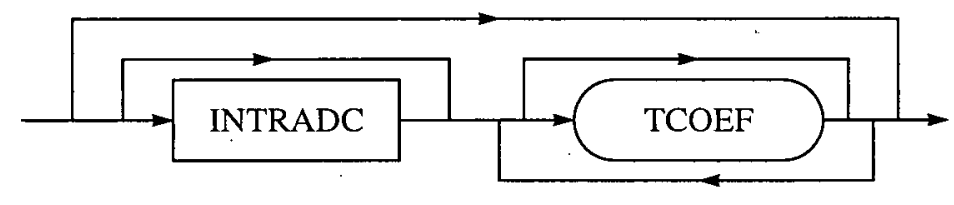

Variable Length Coding

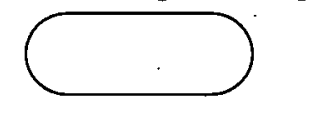

Fig. 2.4 Simplified H.263 Video Bitstream Syntax [21].

transmitted. GOB header starts with Group of Block Start Code (GBSC), followed by Group Number (GN), GOB Frame ID (GFID), GOB Sub-Bitstream Indicator (GSBI), and GOB quantizer information (GQUANT).

Each GOB is sub-divided into Macroblocks in the Macroblock layer [12]. Macroblock header includes Coded Macroblock Indication (COD), Coded Block Pattern (CBP), Macroblock type and Coded Block Pattern for Chrominance (MCBPC), Coded Block Pattern for Bidirectional blocks (CBPB), Coded Block Pattern for Luminance (CBPY), Motion Vector Data (MVD), Motion Vector Data for optional advanced prediction mode $\left(\mathrm{MVD}_{2-4}\right)$, and Motion Vector Data for Bi-directional macroblocks (MVDB). Macroblock mode for Bi-directional blocks 
(MODB) indicates whether CBPB and/or MVDB are transmitted for a Macroblock. Quantizer for the Macroblock is specified in DQUANT.

Lastly, for the Block Layer, a block is composed of $64(8 \times 8)$ DCT-coded residual data. DC coefficient for INTRA blocks (INTRADC) is present for every prediction block that is intracoded. This code is followed by variable length coding representation of Transform Coefficients (TCOEF).

\subsection{Mobile Radio Propagation: Large-Scale Fading}

In this section, we describe the large-scale fading model for the mobile radio channels. Largescale propagation models predicts the average received signal strength for an arbitrary transmitterreceiver separation distance in usually several hundreds or thousands of meters [5]. Average signal strength attenuation in large-scale fading is mainly due to two factors, namely propagation loss and shadowing.

Both theoretical and measurement-based propagation models indicate that average received signal power decreases logarithmically with distance, for both outdoor or indoor radio channels [5]. The attenuation is proportional to $d^{\gamma}$, where $d$ is the distance between two points and $\gamma$ is the path loss exponent. In addition to path loss in a mobile radio link, there also exists the phenomenon of shadowing. Shadowing comes from the diffraction effect on signals due to terrains and obstructive objects that are much larger than the wavelength. It is usually modeled as a random variable with a log-normal distribution [7], [53], [55].

With the overall effect of path loss and shadowing, the signal power $P_{R}$ received is proportional to the transmitted signal $P_{T}$ described as follows [7]: 


$$
P_{R} \propto P_{T}\left(\frac{10^{\left(\frac{\xi}{10}\right)}}{d^{\gamma}}\right)
$$

where $\xi$ is a Gaussian random variable with zero mean and standard deviation $\sigma$ in $\mathrm{dB}, d$ is the distance and $\gamma$ is the path loss exponent. Lee [7] suggests that standard deviation $\sigma$ of $8 \mathrm{~dB}$ and path loss exponent $\gamma$ of 4 be used for urban macrocellular environment.

\subsection{Mobile Radio Propagation: Small-Scale Fading}

In this section, we describe the small-scale fading model for the mobile radio channels. Smallscale fading model characterizes the rapid fluctuation of the received signal strength over very short travel distance (a few wavelengths) or short time duration (on the order of seconds) [5]. It is caused by interference between two or more versions of the transmitted signal arriving at the receiver at slightly different times due to multipath reflections by local scatterers [55]. These multipath waves combine at the receiver antenna to give a resultant signal which can vary widely in amplitude and phase, depending on the distribution of the intensity and relative propagation time of the waves and the bandwidth of the transmitted signal.

\subsubsection{Small-Scale Fading: Parameters and Characterization}

To describe the time dispersive nature of a channel, multipath spread $T_{m}$ is used in the measurement. It denotes the difference in time delay of arrivals from different signal paths in a mobile radio environment due to multipath reflections [5]. For an impulse transmitted at the cell site, by the time this impulse is received at the mobile unit it is no longer an impulse but rather a pulse with a spread width of $T_{m}$. Related closely to the multipath spread is the coherence bandwidth 
$B_{c o}$. It represents the range of frequencies over which two frequency components have a strong potential for amplitude correlation [5]. Coherence bandwidth is defined as the reciprocal of multipath spread $T_{m}$. Another parameter related to the time spreading of a multipath signal is the multipath intensity profile (MIP). It is the average power at the output of the channel as a function of path delays [26]. Different distributions of MIP such as exponential [38], Gaussian [39] and uniform [40] have been used in the literature.

To describe the time varrying nature of the channel in small-scale fading, Doppler spread $B_{d}$ is being used. It denotes the amount of frequency spectrum broadening caused by the time rate of change of the mobile radio channel [26]. Doppler spread $B_{d}$ is a function of the relative velocity of the mobile, and the angle $\alpha$ between the direction of receiver and the direction of arrival of the scattered waves. The dual of Doppler spread in time domain is called the coherence time $T_{c o}$. It designates the time duration over which the channel impulse response is essentially invariant [5]. It is inversely proportional to the Doppler spread $B_{d}$.

When an information-bearing signal is transmitted through the fading channel, if the coherence bandwidth is small in comparison to the bandwidth of the transmitted signal, the channel is said to be frequency-selective [5]. On the other hand, if the coherence bandwidth is large in comparison to the bandwidth of the transmitted signal, the channel is said to be frequency-nonselective or simply flat. For relatively high Doppler spread or short coherence time compared to a symbol duration (e.g. $T_{c o}$ is less than $10 \%$ of $T$ ), the channel is categorized as fast fading [5]. On the other hand, for relatively low Doppler spread (e.g. $B_{d}$ less than $10 \%$ of the original spectrum) or long coherence time compared to a symbol duration, the channel is categorized as slow fading. 


\subsubsection{Small-Scale Fading: Distribution Models}

Empirical results as well as physical reasoning show that the total intensity of each resolvable path is formed by the superposition of radio waves that arrive at the receiver almost simultaneously [22], [24]. Thus, the problem of obtaining the distribution of the signal strength of each multipath coincides with the random phasor problem [29]. Although Rayleigh and Rician distributions are often used in literature for modeling small-scale fading [13], [14], [18]-[21], [27], [42], [43], they only provide the special case solutions of the random phasor problem. In contrast, the Nakagami- $m$ distribution provides a more general solution [35], [36], [37]. Moreover, it is shown in [22] that Nakagami- $m$ distribution provides a better fit for the empirical fading statistics. Due to the above advantages, in this thesis we have adopted the Nakagami- $m$ distribution to model for the small-scale fading.

In the following sub-sections, we will first describe the conventional Rayleigh and Rician fading models. Then Nakagami model will be introduced and its relationship with Rayleigh and Rician distributions will be explained.

\subsubsection{Rayleigh Fading Model}

When there is an absence of line-of-sight wave between the transmitter and receiver, addition of pure scattered waves causes the resultant wave amplitude exhibiting a Rayleigh-distributed behavior. It is well known that the envelope of a complex Gaussian noise signal follows a Rayleigh distribution. The probability density function (PDF) of the Rayleigh distribution is [26]

$$
p(r)=\frac{r}{\sigma_{a}^{2}} e^{-\left(\frac{r^{2}}{2 \sigma_{a}^{2}}\right)}, \quad 0 \leq r<\infty
$$

where $\sigma_{a}$ is the root mean squared.(RMS) value of the received voltage signal, and $\sigma_{a}^{2}$ is the 
time-average power of the received signal. The PDF of the phase of a Rayleigh fading signal follows a uniform distribution around $[0,2 \pi)$.

\subsubsection{Rician Fading Model}

In Rician fading, random multipath components arriving at different angles are superimposed on a stationary dominant signal. As the dominant signal becomes weaker, the composite signal resembles a noise signal which has an envelope that is Rayleigh. The Rician distribution is given as [26]

$$
p(r)=\frac{r}{\sigma_{b}^{2}} e^{-\frac{r^{2}+A^{2}}{2 \sigma_{b}^{2}}} I_{0}\left(\frac{A r}{\sigma_{b}^{2}}\right), \quad A \geq 0, r \geq 0
$$

where $\sigma_{b}^{2}$ is the average power of the fading signal, $A$ is the peak amplitude of the dominant signal, and $I_{0}(\cdot)$ is the modified Bessel function of the first kind and zero-order. The Rician distribution is often described in terms of a parameter $\kappa$ which is defined by the deterministic signal power and the variance of the multipath signal. It is given by [36]

$$
\kappa=\frac{A^{2}}{\sigma^{2}} .
$$

The parameter $\mathrm{K}$ is known as the Rician factor and completely specifies the Rician distribution. As $A \rightarrow \infty, \kappa \rightarrow \infty$, and as the dominant path decreases in amplitude, the Rician distribution degenerates to a Rayleigh distribution.

The phase of a Rician fading signal has a PDF of [58]

$$
p(\Theta)=\frac{e^{-\kappa}}{2 \pi}+\frac{\sqrt{\kappa} \cos \Theta \exp \left(-\kappa \sin ^{2} \Theta\right)}{2 \sqrt{\pi}} \cdot[2-\operatorname{erfc}(\sqrt{\kappa \cos \Theta})]
$$




\subsubsection{Nakagami- $m$ Distribution}

The PDF of Nakagami- $m$ distribution was deduced by Nakagami from his large scale experiments on rapid fading in high frequency long distance propagation and has the form of [35]

$$
p(r)=\frac{2 m^{m} r^{2 m-1}}{\Gamma(m) \Omega^{m}} e^{-(m / \Omega) r^{2}}
$$

In (2.6), $\Gamma(m)$ denotes the Gamma function, defined as $\Gamma(m)=\int_{0}^{\infty} t^{m-1} e^{-t} d t$ for $m>0$. The parameter $\Omega$ is the second moment of $r$, or the mean power, defined as $\Omega=E\left[r^{2}\right]$, where $E[\cdot]$ is the average function. The parameter $m$ is the fading figure characterizing the severity of the fading, and is equal to the inverse of the normalized variance of $r^{2}$ as [35]

$$
m=\frac{E^{2}\left[r^{2}\right]}{E\left[\left(r^{2}-E^{2}\left[r^{2}\right]\right)^{2}\right]} \geq \frac{1}{2}
$$

As for special cases, $m=1$ corresponds to purely diffusive scattering or Rayleigh fading. $m \rightarrow \infty$ corresponds to the non-fading condition [23].

The Rician distribution can also be closely approximated by the Nakagami- $m$ distribution. The approximation can be obtained through functional relationship of the parameters of the two distributions $\kappa$ and $m[36]$

$$
m=\frac{1}{1-\left(\frac{\kappa}{1+\kappa}\right)^{2}}
$$

As shown by (2.8), the relationship between $m$ and $\kappa$ is not strictly linear. However, an accurate linear approximation can be obtained for $\kappa \geq 2$ as [36]

$$
m=s_{m} \kappa+m_{0}
$$

where $s_{m} \approx 0.5$ and $m_{0}=0.7622$ for $\kappa \sim\{2-100\}$. From (2.9), it is observed that $m$ is a 
similar parameter as $\kappa$ in terms of physical interpretation, i.e. it is the amount of specular power in the received signal with respect to scattered power.

\subsection{Spread Spectrum Modulation Techniques}

In this section, we explain the basic concept of spread spectrum modulation techniques. Spread spectrum techniques transform a signal with bandwidth $B$ into a noise-like signal of much larger bandwidth $B_{s s}$, which usually is of several orders of magnitude greater than the original signal bandwidth [3], as illustrated in Fig. 2.5. The amount of spreading is measured as the ratio

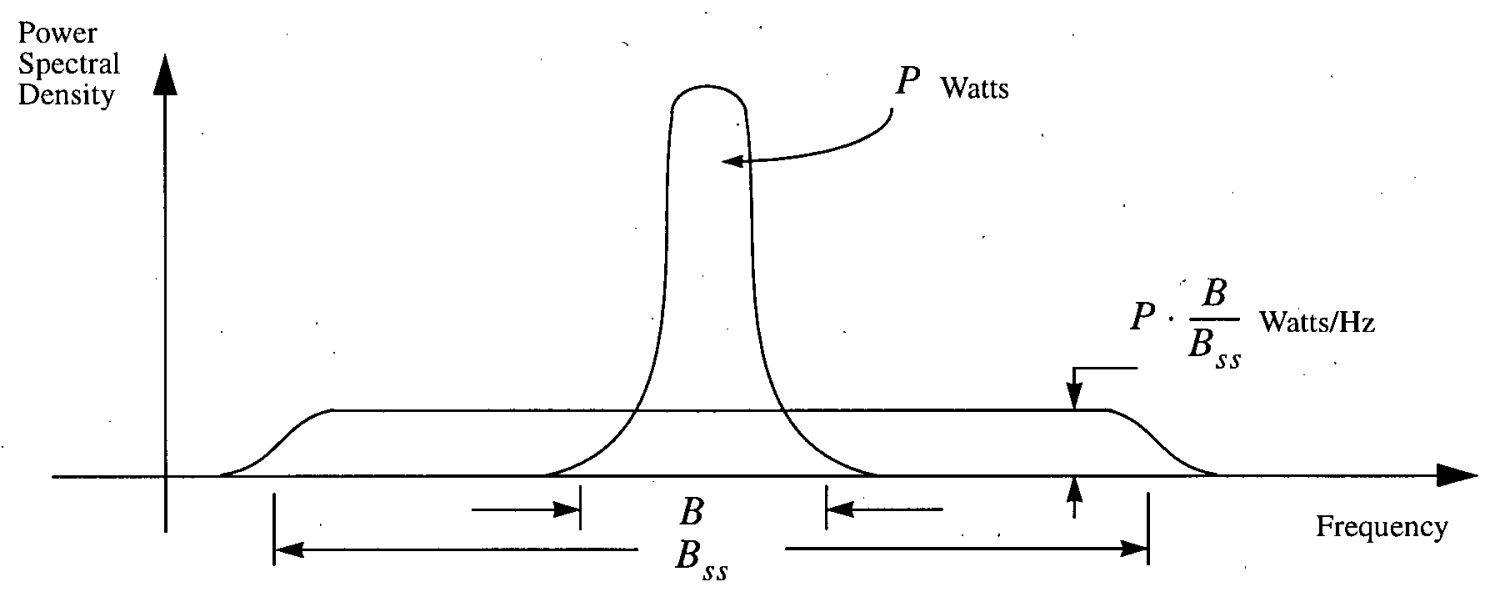

Fig. 2.5 Signal Spectra Before and After Spreading [3].

$N=B_{s s} / B$ called the processing gain. As a result, the power of the radiated spread spectrum signal is spread over a much broader bandwidth, while its power spectral density is correspondingly reduced by the same proportion.

Each spread signal is pseudorandom and has noise-like properties when compared with the digital information data [5]. The spreading waveform is controlled by a pseudo-noise (PN) 
sequence, which is a binary sequence that appears random but can be reproduced in a deterministic manner by the intended receiver. Spread spectrum signals are demodulated at the receiver through cross-correlation with a locally-generated version of the pseudorandom codes. Crosscorrelation with the correct PN sequence despread the desired spread spectrum signal and restores the modulated message in the same narrowband as the original data, whereas cross-correlating the signal from other users result in small amount of wideband noise at the receiver output.

There are many distinct advantages of spread spectrum waveforms. The most important one is its inherent interference mitigation capability [5]. Since each user is assigned with a unique PN sequence which has very little correlation with others, the receiver can distinguish the intended user from the rest based on their spreading codes, even though they all share the same frequency spectrum and bandwidth. Moreover, since narrowband interference affects only a small portion of the spread spectrum signal, their effects are negligible for spread spectrum signal. Furthermore, a direct sequence CDMA cellular system can apply a universal one-cell frequency reuse pattern [1]. Hence, there is no need for complicated frequency planning or frequency guardband assignment as in TDMA or FDMA schemes. As a result, it greatly simplifies radio resource management and minimizes wastage.

Wide bandwidth characteristics of spread spectrum signal also provides effective mitigation of multipath fading by introducing multipath diversity [1]. Since its bandwidth is usually much wider than the channel coherent bandwidth, the channel is categorized as frequencyselective [5]. At any given time; only a small portion of the wideband signal will undergo fading, whereas the rest of it remains high in signal-to-noise ratio facilitating correct signal detection. Besides this resistant nature to multipath fading, spread spectrum systems can take advantage of the frequency-selective characteristics of the signal by resolving the multipath components using 
a RAKE receiver for improved performance [6], the structure of which will be presented in the next section.

\subsection{The RAKE Receiver}

In this section, we describe the structure of a RAKE. receiver used in CDMA systems. In a multipath frequency-selective fading channel, conventional modulation techniques require equalizer to cancel intersymbol interference between adjacent symbols [5]. For spread spectrum modulation used in CDMA systems, however, the situation is quite different. For direct sequence spread spectrum signals, the spreading codes are designed to have very low correlation between successive chips. Thus, propagation delay spread in the radio channel merely provides uncorrelated multiple versions of the transmitted signal at the receiver, on condition that these multipath components have time separations of more than one chip duration. In a CDMA system, it is not only that no equalization is required, but the receiver can actually gain performance from the multipath characteristics of the channel by combining those multipath signal components constructively.

Such a CDMA receiver which uses multipath diversity is referred to as the RAKE receiver, and was first proposed by Price and Green [6]. A RAKE receiver consists of a bank of correlators called fingers, each of which correlates to a particular multipath component of the desired signal. The correlator outputs are weighted according to their relative signal strength to achieve maximal ratio combining. For a CDMA system, the RAKE receiver acts essentially as a diversity receiver, where the diversity is provided by the fact that the multipath components are practically uncorrelated from one another when their relative propagation delays exceed one chip duration. If the output from one correlator is corrupted by fading, the others may not be, and the 
corrupted signal may be discounted through the weighting process. The adverse effect of fading can thus be greatly reduced by diversity provided by combination of all finger statistics before receiver output decision is made.

Fig. 2.6 illustrates a RAKE receiver structure employing $M$ fingers [5]. In the receiver, each finger of the RAKE correlates to a portion of the received signal $r(t)$ which is delayed by at least one chip in time from the other fingers. The output of the $M$ correlators are denoted as $Z_{1}, Z_{2}, \ldots, Z_{M}$. They are weighted by $\alpha_{1}, \alpha_{2}, \ldots, \alpha_{M}$ correspondingly, whose coefficients are directly proportional to the instantaneous power from each correlator output. The weighted output of the $M$ correlators are then summed up as $Z^{\prime}$ and integrated to produce the decision variable $Z$ before being sent to the decision device for symbol detection.

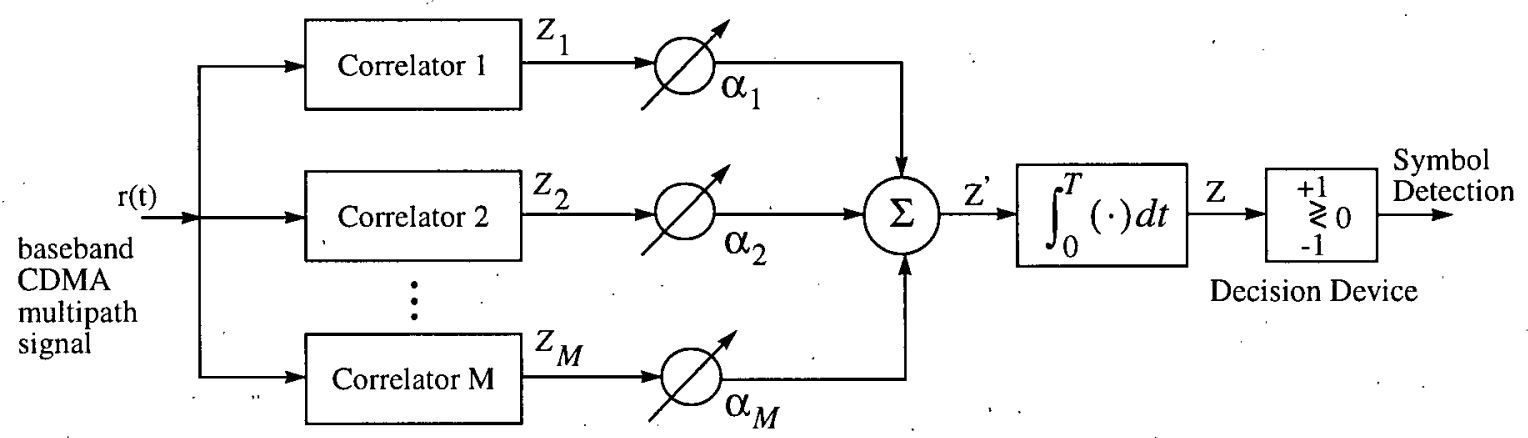

Fig. 2.6 An $M$-finger RAKE Receiver [5].

\subsection{IS-95 Code Division Multiple Access System}

The communication system model employed in this thesis is based on the Interim Standard 95 (IS-95) “Mobile Station-Base Station Compatibility Standard for Dual Mode Wideband Spread Spectrum Cellular System" originally issued by the U.S. Electronics Industries Association (EIA) 
[9]. The following sub-sections describe the relevant topics of the standard for this thesis.

\subsubsection{CDMA Forward Link}

Fig. 2.7 shows the forward (down-link) traffic channel modulation of a IS-95 CDMA system [9].

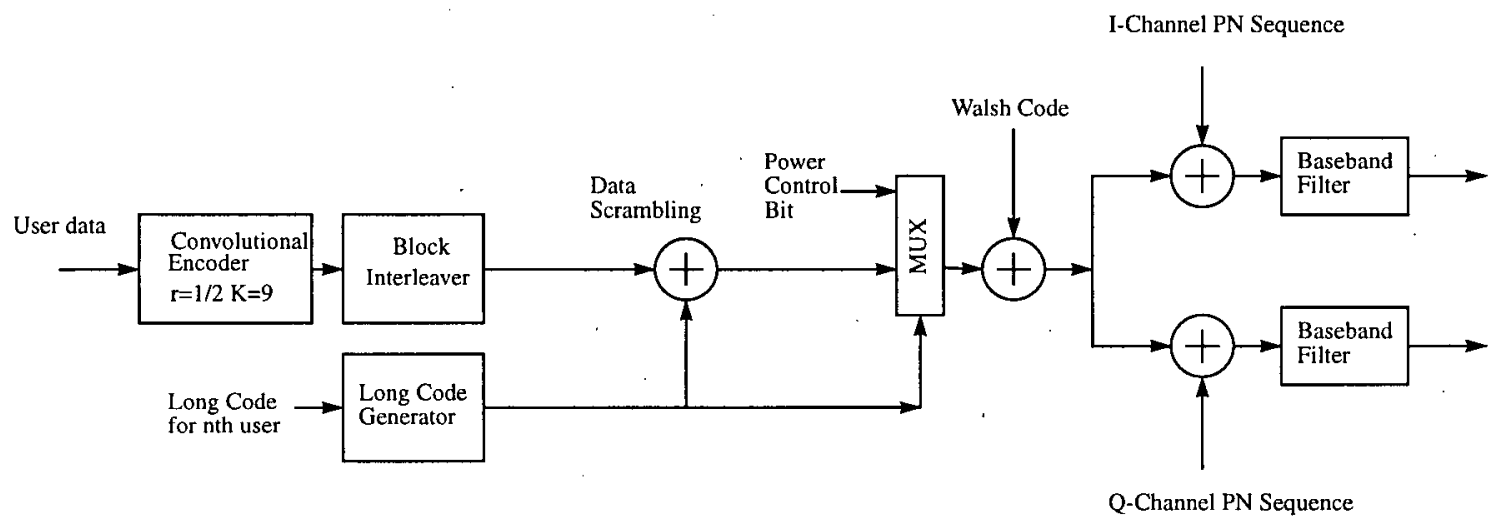

Fig. 2.7 Forward CDMA Channel [9].

The information transmitted is convolutionally encoded to provide the capability of error detection and correction at the receiver. The code used has a constraint length of nine, $K=9$, and a code rate of one-half, $r=1 / 2$. The output of the convolutional coder is then block interleaved using a 24 x 16 block interleaver over a 384-symbol interval to reduce bursty errors resulting from mobile radio channels. The data symbols are then scrambled by a user-specific long code with a period of $2^{42}-1$ chips. Data scrambling serves the purpose of user identification and security [55]. To minimize the average BER for each user, IS-95 strives to force each user to provide the same power level at the base station [56]. Power control commands are sent to each subscriber unit through the Power Control Bit. Following data scrambling is the orthogonal covering. Each data symbol is orthogonally spread by one of the 64 orthogonal Walsh codes, 
which are binary sequences completely orthogonal to each other [5]. Signals from different users within a cell are distinguished by their Walsh codes. After the orthogonal covering, data symbols go through quadrature spreading where a pair of short binary PN sequences (the basic codes) with a period of $2^{15}-1$ chips is used in the in-phase and quadrature branches. All base stations share a common quadrature pair of PN codes, but each are assigned a unique time offsets value [56]. Signals from different cells are distinguished by the time offsets from the basic codes. Following quadrature spreading, each of the two branches is filtered separately with a finite impulse response (FIR) filter prior to carrier modulation.

The forward link consists of a pilot channel, a synchronization channel, up to seven paging channels, and up to sixty-three forward traffic channels [55]. Among them, the pilot channel is one of the most important aspects of the forward link signal design. Each cell-site transmits a pilot tone which is used as a coherent carrier reference for demodulation by all mobile receivers [56]. The pilot channel signal is transmitted at a relatively higher power level than the other channels (e.g. synchronization channel, paging channels and traffic channels) so that extremely accurate tracking can be achieved. Specifically, it is simply a constant-level signal that is modulo-2 added with the all-zeros Walsh code and sent over the air after quadrature spreading. The mobile station synchronizes with the nearest base station by detecting the pilot tone with the strongest signal level. It then determines the identity of the base station by the time offset from the basic codes, a unique value for every cell in the system. After synchronization, the pilot signal is used as a coherent carrier phase reference for demodulation of the other signals from this base station. Remaining synchronization details and other system information is sent to the mobile station through the synchronization channel. Once the synchronization channel has been received, the mobile station uses one of the paging channels to receive other system information and paging 
commands.

\subsubsection{Direct Sequence Spreading Codes}

The ideal spreading code would be an infinite sequence of equally likely random binary digits [57]. Unfortunately, in order to despread the signal, we need to have the same version of the spreading code at both the transmitter and receiver. It implies the need for infinite storage at both ends, which is clearly impossible. Alternatively, periodic pseudo-noise (PN) codes are used in spread spectrum systems. These are codes that can be easily generated by means of shift registers and have noise-like behavior. IS-95 CDMA systems use maximal-length codes ( $m$-sequences) for part of the spectrum spreading (see Fig. 2.7). For a multiple-access system, it is important to minimize interference among signals from different users. Therefore, in IS-95 CDMA spectrum spreading scheme, a class of orthogonal codes called the Walsh codes is also used to provide nearly perfect isolation between the multiple-user signals transmitted by a base station [56].

We will discuss each of these codes in the following sub-sections. Their unique properties, either desired or undesired for a CDMA system, will be explored. We will also describe how the IS-95 standard employs the method of code concatenation to combine the best of both codes.

\subsubsection{Maximal-Length Sequences}

The PN sequences used in IS-95 are $m$-sequences generated by 15 linear shift registers based on the characteristic polynomials given in [9] with a period of 32767 chips:

$$
\begin{aligned}
P_{I}(x) & =x^{15}+x^{13}+x^{9}+x^{8}+x^{7}+x^{5}+1 \\
P_{Q}(x) & =x^{15}+x^{12}+x^{11}+x^{10}+x^{6}+x^{5}+x^{4}+x^{3}+1
\end{aligned}
$$

Periodic autocorrelation function is an important characteristic of a periodic PN sequence, and it 
is defined as [26]

$$
\phi(j)=\sum_{i=1}^{n}\left(2 b_{i}-1\right)\left(2 b_{i+j}-1\right), \quad 0 \leq j \leq n-1
$$

where $n$ is the period and $b_{i}$ is the $i$ th binary bit of the sequence. It would be ideal to have sequences that have periodic autocorrelation values of $\phi(0)=n$ and $\phi(j)=0$ for $1 \leq j \leq n-1$. During code despreading, it would provide maximum correlation value for matched code detection while rejecting any other offset sequences by the zero correlation value. In case of $m$ sequences, the periodic autocorrelation function is [26]

$$
\phi(j)=\left\{\begin{array}{c}
n, \quad j=0 \\
-1, \quad 1 \leq j \leq n-1 .
\end{array}\right.
$$

As the period $n$ gets larger, the off-peak values become insignificant compared to the peak value. Thus, $m$-sequences are almost ideal in terms of their autocorrelation function. However, $m$ sequences are not practical to be used alone for CDMA because the periodic cross-correlation function between any pair of the $m$-sequences of the same period can have relatively large peaks [34]. This is clearly undesirable in CDMA systems since it will increase interference which in turn reduce capacity. Although it is possible to select a small subset of $m$-sequences that have relatively smaller cross-correlation peak values, the number of such sequences in the set is usually too small for CDMA applications [26].

\subsubsection{Walsh Sequences}

Walsh codes provide orthogonal covering for IS-95 CDMA system [5]. These Walsh functions comprise of binary sequences generated from a dimension-64 Hadamard matrix. Each Walsh sequence is strictly orthogonal to one another. During spreading, each information symbol is 
replaced by one of the 64-bit long Walsh codes which is uniquely assigned to each user within a cell.

In the absence of multipath, the Walsh codes provide perfect orthogonal channelization for users on the forward link within the cell. However, the orthogonality breaks down in a multipath mobile environment where multipath delays introduce inhomogenous auto-correlation and crosscorrelation characteristics [33]. If Walsh sequences are used alone, the value of auto-correlation and cross-correlation of orthogonal sequences can be very high for certain time delays. Moreover, not all of the Walsh sequences has wideband spectral characteristics as desired in spread spectrum [5]. These undesirable effects can be mitigated by code concatenation with a PN sequence as is done in IS-95 CDMA systems.

\subsubsection{Concatenated Walsh/PN Sequence}

IS-95 CDMA systems use code concatenation scheme with Walsh functions and $m$-sequences, which combines the desirable properties from both codes [9]. Each of the 64-chip long Walsh sequence is modulo- 2 added with a chip from the 32767 -chips long $m$-sequence. Since the length of these two sequences are relatively prime to each other, it allows every chip from the $m$ sequence to occur at the beginning of some data bit during the concatenation.

The resulting concatenated Walsh/ PN codes provide orthogonality between multiple users within the same cell in a single path propagation environment, while reducing the inhomogenous behavior of Walsh cross-correlation due to non-zero time delays in multipath environment [32]. Moreover, concatenation scheme reduces interference between mobiles that use the same Walsh code in different cells. Furthermore, it provides the desired wideband spectral characteristics for direct sequence spread spectrum signals in CDMA systems. We will provide numerical results to 
verify the advantage of using concatenated codes over non-concatenated codes in Chapter 4 .

\subsection{Conclusions}

In this chapter, we presented some of the essential background material for the better understanding of this thesis. We described the H.263 video coding scheme. Then, different wireless channel models were explained. Furthermore, spreading spectrum techniques and RAKE receiver mechanism were illustrated. Lastly, the IS-95 standard and its spreading codes were discussed. 


\section{Chapter 3 MODIFIED H.263 VIDEO CODEC}

\subsection{Introduction}

One inherent problem with any communications system is that information may be altered or lost during transmission due to channel noise, interference and distortion. The effect of such information loss can be devastating for the transport of compressed video because any damage to the compressed bitstream may lead to objectionable visual distortion at the decoder. Several techniques have been suggested to limit the damage of channel errors for H.263 video compression [13]-[21], [45]-[50]. In general, they fall into three categories namely, Forward Error Correction (FEC) [14], [21], [45], [50], Automatic Repeat reQuest (ARQ) [16], [17] and a combination of both [13], [18], [19], [20], [46], [49]. For schemes utilizing ARQ, retransmission of corrupted frames introduces additional delay. This makes the use of ARQ for voice oriented applications very limited because a round-trip delay of less than $300 \mathrm{~ms}$ is required [17]. Therefore, for realtime applications, FEC approach is more suitable, especially if round-trip delay is considerably long.

In this chapter, we propose a modified H.263 codec which is based upon a combination of FEC coding scheme and periodic frame refresh procedure. The proposed modification is compatible with the H.263 standard to improve video quality for low bit-rate video transmission over error-prone wireless channels. In Section 3.2, we will first investigate the impact of video bitstream errors on H.263 video quality. Then, in Section 3.3 and 3.4, we will describe the details of the proposed scheme. Lastly, in Section 3.5, numerical results for the video codec performance in a AWGN channel is presented. 


\subsection{Effects of Errors on H.263 Video}

Generally, the severity of bit errors to video quality depends on the spatial and temporal location of the error. Since H.263 coding scheme uses prediction frame statistical redundancy removal coding techniques, temporal and spatial error propagation problems are inherent. Huffman coding causes spatial error propagation problem, whereas motion compensation causes temporal error propagation problem. Based on some recently reported results [21], [45], [50], the effects of transmission errors on H.263 video can be summarized as follows:

- Errors in video headers can cause major damage, especially for headers in higher hierarchical layer of H.263 coding such as the Picture Layer and the Group of Blocks Layer.

- Errors propagate in spatial domain due to improper decoding of variable length codes; The Picture and Group of Block Layer headers stop error propagation in spatial domain by providing start codes.

- Errors propagate among Prediction frames in the temporal domain; INTRA frames stop the propagation by coding the picture frame independently.

To maximize video quality, while avoiding time delay for real-time applications and minimizing channel coding redundancy and complexity, we propose a selective FEC coding scheme combined with an INTRA frame forced update mechanism.

\subsection{Selective FEC Coding}

The family of $\mathrm{BCH}$ codes are powerful linear block codes for which efficient decoding algorithms exists [57]. This family of codes contains codes of many rates and a wide range of error correcting capability. We adopt $\mathrm{BCH}$ codes because of their strong error-correcting power and relatively low 
complexity. An appropriate block interleaver is needed to transform the bursty error statistics of the mobile channel into Gaussian-like statistics required by the shorter $\mathrm{BCH}$ codes. As mentioned in Section 2.7, such block interleaver is included in the IS-95 forward link standard.

Since IS-95 CDMA system uses half-rate convolutional coding on all transmitted data, it provides the base layer FEC protection. Considering the impact of errors on the H.263 video bitstream and in order to minimize the redundancy introduced by the coding, we choose to introduce two extra FEC protection to selected bits of the upper two hierarchical layers of the H.263 bitstream syntax. For the lower two hierarchical layers, because of the lack of start code for synchronization and of the variable nature of the sequence lengths, the positions of their bits are unknown. Thus, in order to protect any selected bits from these two layers, extra information bits have to be introduced to indicate the positions and lengths of those chosen bits. However, these extra information bits, which are as important as the chosen bits we would like to protect, do not have any extra FEC coverage. Therefore, the effort to selectively protect bits in the lower two hierarchical layers may not be worthwhile because it introduces equally error-sensitive information bits during the process.

Assuming that a QCIF sequence (176 x 144 luminance resolution, 4:1:1 chrominance subsampling) is transmitted, FEC1 provides error protection for the important header bits in Picture layer of both INTRA and Prediction frame which include PSC, GN, TR, PTYPE, PQUANT, CPM, PEI. They add up to a total of 50 bits and a $(71,50,3) \mathrm{BCH}$ codeword is assigned. 21 bits of redundancy are introduced for correcting up to three errors. FEC2 provides error protection for header bits in GOB layer which includes GBSC, GN, GFID, GQUANT. These 29 bits are protected by a $(41,29,2) \mathrm{BCH}$ codeword. 12 bits of redundancy are introduced for correcting up to two errors. 


\subsection{Forced INTRA Frame Update}

The major compression achieved by low bit rate encoders such as H.263 is mostly due to the removal of temporal redundancy through motion compensation. Therefore, it is necessary for the encoder to emulate the decoder at the transmitter. If the information available to this decoder and the decoder used at the receiver are not the same, the quality of the reconstructed picture-frame can degrade considerably. Moreover, temporal mitigation of these reconstruction errors can affect the quality of the subsequently reconstructed picture frames. If the reconstructed signal is degraded due to some channel errors, subsequent reconstruction of error-free transmissions may also be incorrect. Therefore, it is apparent that to ensure high quality of transmission, effects of errors must not propagate too far beyond the temporal interval of the channel errors. One solution to this is to apply forced update of the prediction frames by INTRA frames periodically. Considering the trade-off between bit usage and overall video quality, we have chosen to apply INTRA frame update in every 10 prediction frames.

\subsection{Numerical Results for the AWGN Channel}

The implementation of the modified H.263 video codec was based on the Test Model Near-term (TMN) version 2.0 software platform developed by Telenor R\&D, Norway. We consider the transmission of 100 QCIF video frames of the well known sequence Miss America (Fig. 3:1) over AWGN channels at a nominal rate of $64 \mathrm{kbits} / \mathrm{s}$. The overhead introduced by FEC coding is about $1.1 \%$. Results with average bit error rates of $10^{-3}$ to $10^{-4}$ are considered. The average Peak Signal-to-Noise Ratio (PSNR) of weighted luminance and chrominance components is used as a measure of objective quality, and is given by 

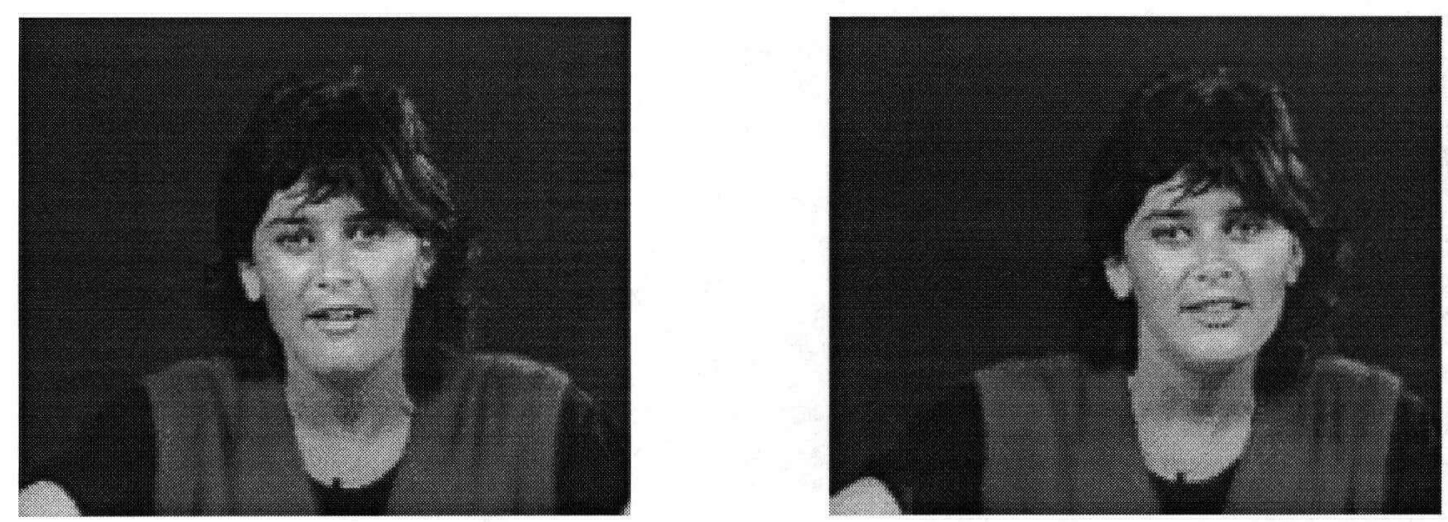

Fig. 3.1 Sample Frames of Miss America Video Sequence (Frame \#0 and 90)

$$
P S N R=(P S N R)_{Y}+0.3(P S N R)_{C_{b}}+0.3(P S N R)_{C_{r}}
$$

where PSNR of each component is calculated as

$$
(P S N R)_{x}=10 \log \frac{1}{M} \sum_{i=1}^{M} \frac{255^{2}}{\left(o_{i}^{x}-r_{i}^{x}\right)^{2}}
$$

In (3.2), $M$ is the number of video frames, $o_{i}^{x}$ and $r_{i}^{x}$ are the amplitudes of the original and reconstructed luminance $(Y)$ or chrominance $\left(C_{b}, C_{r}\right)$ component values, respectively. The conventional method of calculating PSNR is to take luminance components into account only [19], [20]. For monochrome video, it is probably a sufficient measure to calculate the objective video quality. However, for present and future video communication applications, full color video is more likely to be transmitted over the networks. Since both brightness and colors of the video frames affect human's subjective judgement of the video quality, it is thus logical to take a weighted average of both luminance and chrominance components of the video frames into account when calculating the PSNR for a better measurement of the overall video quality. Notice that every data point is generated by taking the ensemble average of 20 simulation runs using different random seeds.

As shown in Fig. 3.2, the performance of the modified H.263 video codec is superior to 


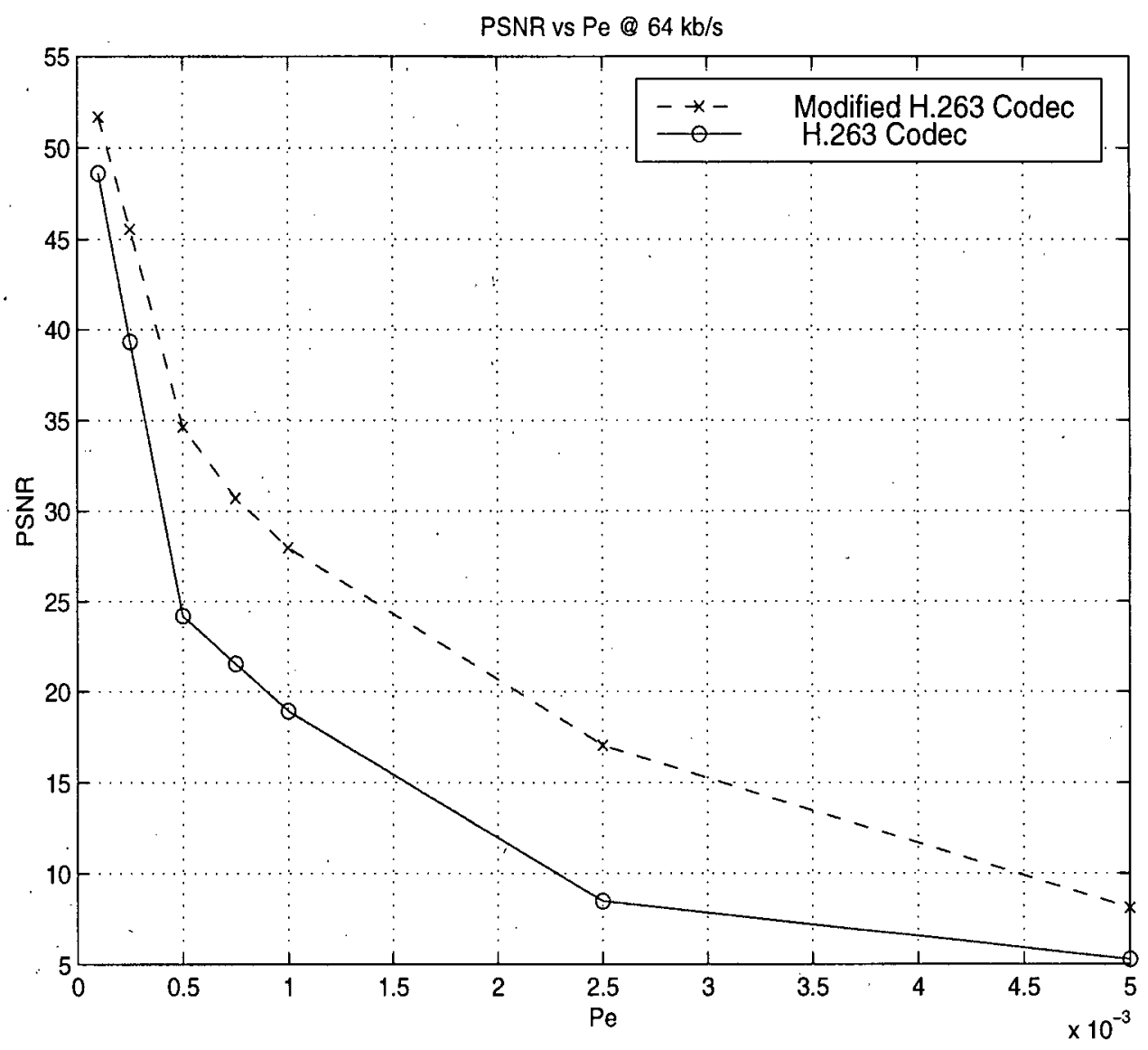

Fig. 3.2 Video Performance of Modified H.263 Codec

that of the original version. Improvement in PSNR ranges from $3 \mathrm{~dB}$ to $10 \mathrm{~dB}$ depending upon the values of error probability $P_{e}$. The gain in performance becomes smaller for lower $P_{e}$ because the loss in video quality due to FEC redundancy is greater than the gain from protected bits being hit by errors. In the other end of higher $P_{e}$ values it is observed that the gain in performance from the proposed scheme becomes smaller again. It is because in severe error condition, the video performance degrades to a point where FEC can provide very little help to recover the damage.

In Fig. 3.3, we show the frame-to-frame performance of both coding schemes at $P_{e}=5 \times 10^{-3}$ compared to error-free condition. Again, it can be observed that the modified 
coding scheme offers better performance in most of the frames, thus resulting in better overall PSNR value. The step-like shape of the modified H.263 codec curve (solid line) is because of the forced INTRA frame refresh mechanism occurs in every ten frames.

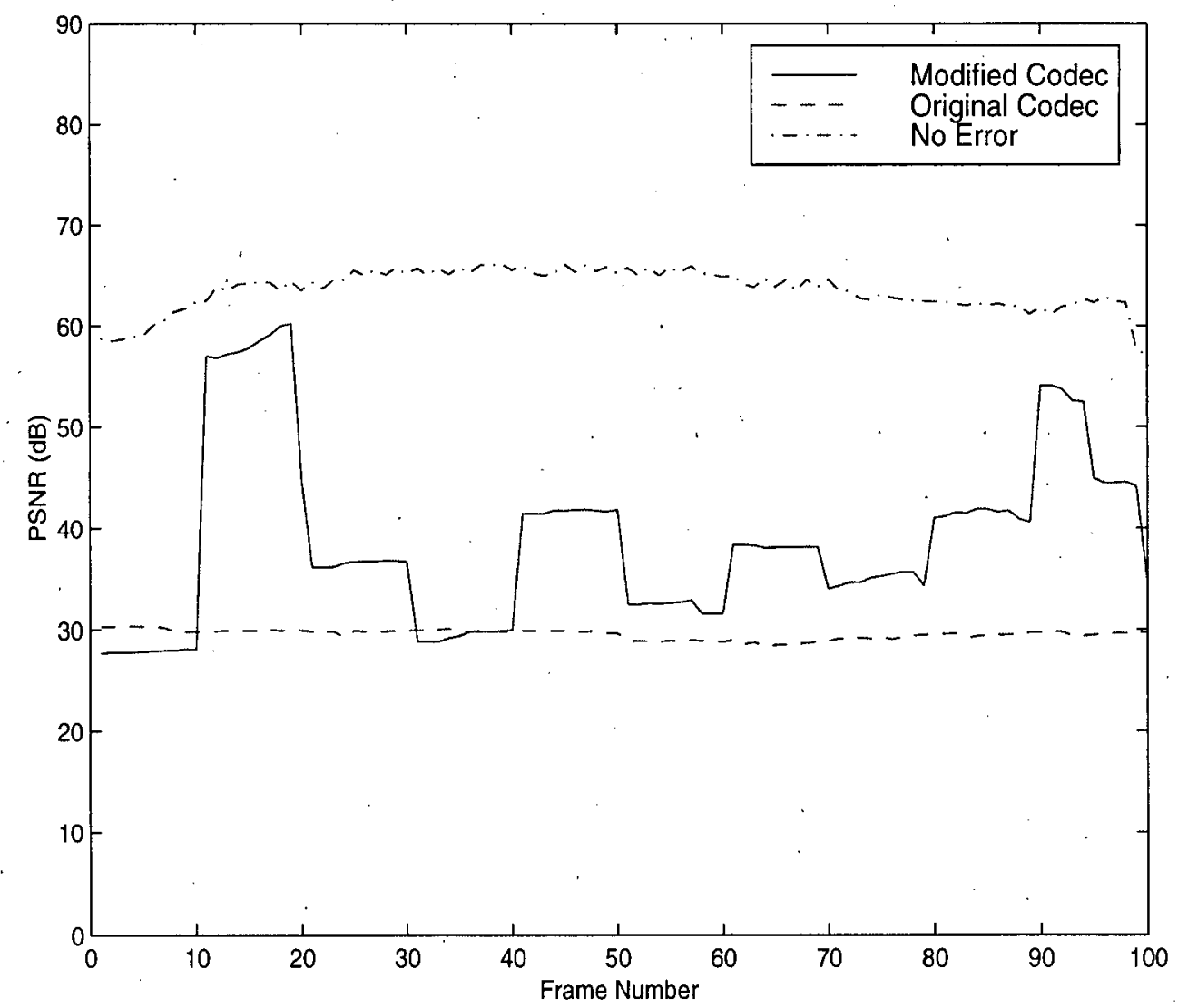

Fig. 3.3 Frame Performance of the Modified H.263 Codec

\subsection{Conclusions}

In this chapter, we discussed the impact of errors on unprotected H.263 bitstream. Then, we proposed a modified encoding scheme which uses selective FEC protection for the higher hierarchical layer bits and forced INTRA frame refresh mechanism to improve performance in error- 
prone wireless environment. We introduced a weighted luminance and chrominance PSNR measurement to better quantify the objective video quality. The chapter was concluded with numerical results which showed the improved PSNR performance of the modified codec over the original one. 


\section{Chapter 4 FORWARD LINK VIDEO TRANSMISSION IN SINGLE-CELL CDMA SYSTEMS}

\subsection{Introduction}

The purpose of this chapter is to investigate the PSNR performance of video transmission over a single-cell DS-SS CDMA forward link under correlated Nakagami fading channel conditions. We first evaluate the BER performance of a CDMA forward link operating in a Nakagami fading environment by employing Gaussian approximation method. The numerical results are then compared with those obtained from the Monte Carlo simulations. For the mobile channel simulations, a correlated Nakagami fading channel simulator is implemented in software. The modified H.263 codec proposed in the previous chapter is integrated with the IS-95 based CDMA system for video transmission performance evaluation.

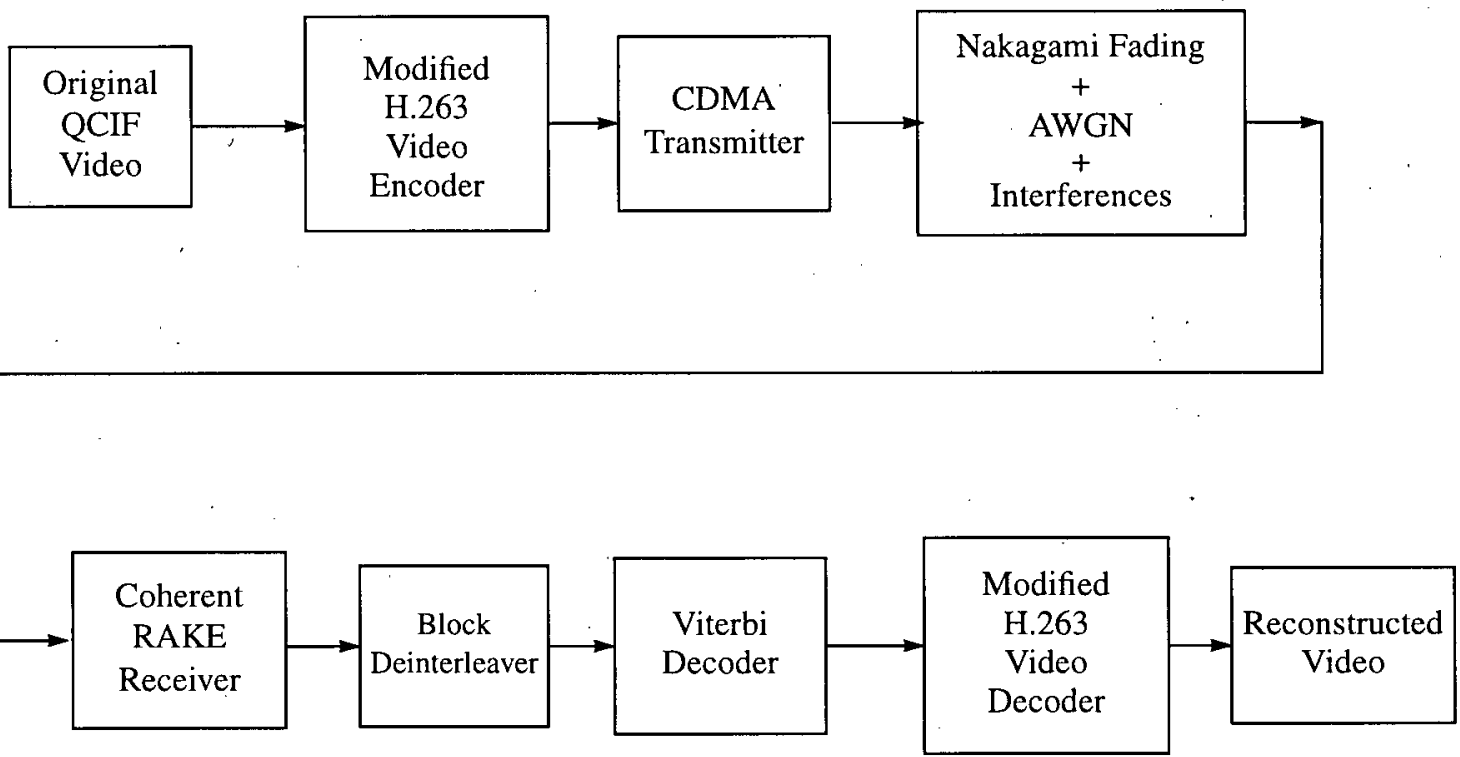

Fig. 4.1 Simulated DS-SS CDMA Forward Link Video Transceiver System Model 
Fig. 4.1 illustrates the block diagram of the simulated video transceiver system model for the forward link of a DS-SS CDMA system through Nakagami fading channels. The uncompressed video sequence in QCIF format is first encoded by the modified H.263 encoder. Then, the compressed video data are sent to the CDMA transmitter located at the base station. The CDMA transmitter consists of convolutional coder, block interleaver and direct sequence spectrum spreading sub-blocks. Details of the direct sequence spread spectrum CDMA model are given in Section 4.2: The signal is modulated using binary phase shift keying (BPSK). Next, the transmitted signal goes through the CDMA mobile channel with Nakagami fading characteristics, and is corrupted by AWGN, self-noise interference and multiple-access interference. Details of the channel model and the Nakagami fading software simulator will be described in Section 4.3 and 4.4, respectively. Following the channel, a coherent RAKE receiver located at the handset is used to resolve the multipath signal through the aid of a pilot tone. The coherent RAKE receiver model will be discussed in Section 4.5. The recovered symbols are then deinterleaved followed by hard-decision Viterbi decoding. Finally, the decoded bits are decompressed by the modified H.263 video decoder to obtain the reconstructed video sequence. In Section 4.6, we first establish the mathematical expressions for the single-cell CDMA forward link system model. To obtain the BER performance for the system, we use both an analytical approach and the Monte Carlo simulation method. In Section 4.7, the Gaussian approximation is employed in the analysis to obtain the BER performance for the forward link. In Section 4.8, we explain the Monte Carlo method and describe the computer simulation model used for the overall system performance evaluation. The numerical results obtained from analytical and computer simulation methods are then compared in Section 4.9. In Section 4.10, we integrate the modified H.263 video codec with the proposed CDMA system and present the numerical results of video transmission performance 
obtained from computer simulations under different system conditions. Finally, the chapter is completed by its conclusions which are presented in Section 4.11 .

\subsection{Direct Sequence-Spread Spectrum (DS-SS) CDMA System Model}

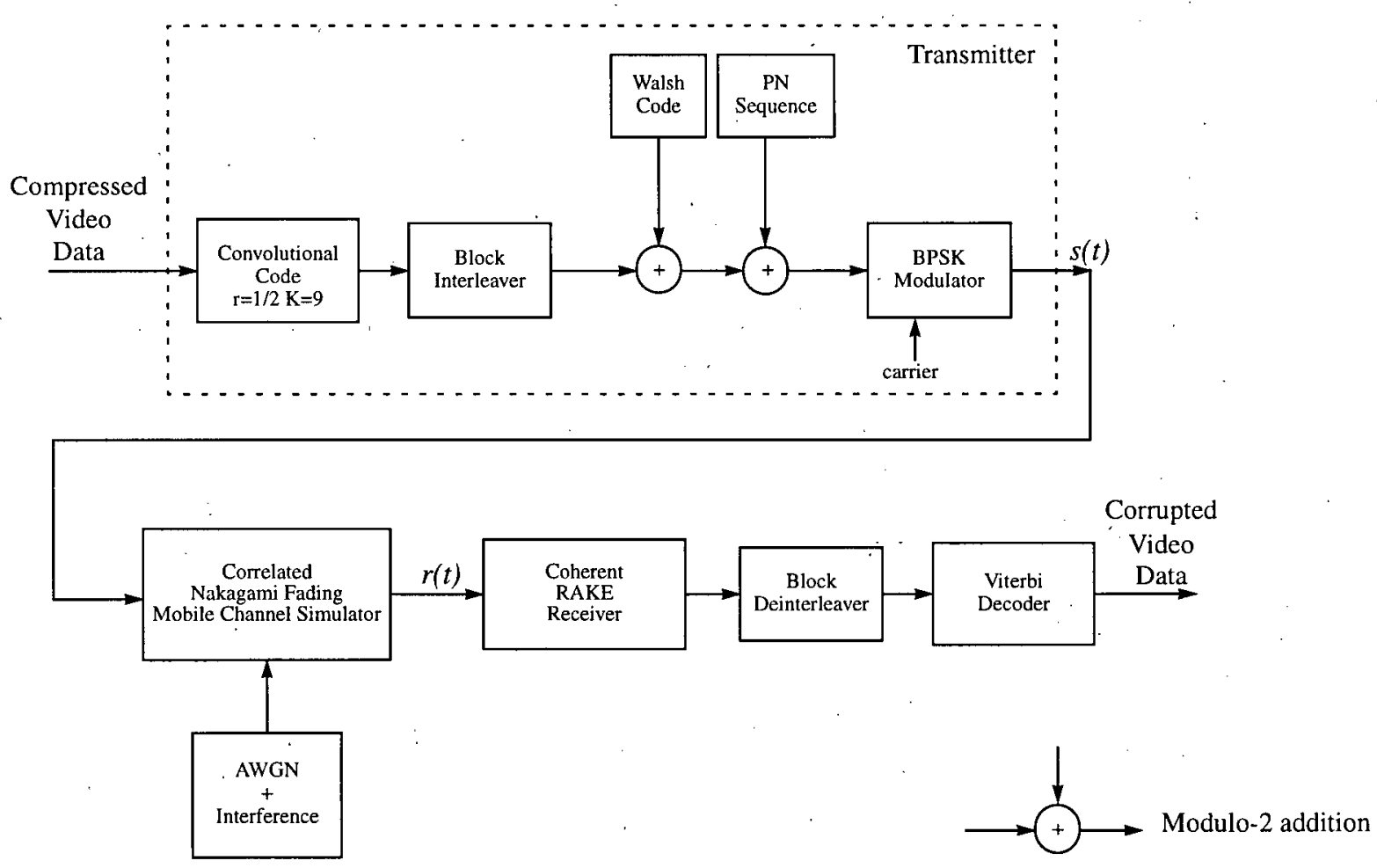

Fig. 4.2 Simulated DS-SS CDMA System Model

Fig. 4.2 illustrates the block diagram of the DS-SS CDMA system model which was considered in this thesis. As per IS-95 standard, the encoded video data is half-rate convolutionally encoded with constraint length of $K=9$. The coding process is described by generator vectors $G_{0}$ and $G_{1}$ which are 753 (octal) and 561 (octal), respectively. After convolutional coding, symbols are interleaved by a block interleaver of size $24 \times 16$ to randomize bursty errors resulting from the multipath fading channels. The symbols are then spread by a concatenated Walsh/ PN sequence. Each symbol is first replaced by one of the sixty-four orthogonal Walsh codes of length 64 chips, 
which is uniquely assigned to each user. Then, modulo-2 addition (concatenation) is performed on each chip with a PN sequence of period $2^{15}-1$ chips. The resulting concatenated codes retain orthogonality among users, while reducing cross-correlation surges among non-concatenated Walsh codes. We assume the forward link to be chip-synchronized so that the signals can take advantage of orthogonal covering by the Walsh sequences. The data are finally BPSK modulated before transmitted through the channel.

For the mobile CDMA channel, we model the fading statistics as Nakagami- $m$ distributed. In addition to fading and AWGN, the transmitted signal is also corrupted by self-noise interference and multiple-access interference. Self-noise contributes interference which originates from the sidelobes of the autocorrelation function of the spread spectrum code assigned to the reference user. Multiple-access interference is caused by the cross-correlation of the spread spectrum codes among reference user and other simultaneous system users in a multipath signal environment.

At the receiver end, we employ a coherent RAKE receiver to resolve multipaths for improved BER performance. A number of correlators are assigned to capture the different multipath components. It is assumed that the base station continuously transmits a pilot signal which is used by the mobile receiver to acquire synchronization as well as to make estimation of the channel impulse response. The output of each finger is maximally-ratio combined before being sent to the decision device. The received data are then deinterleaved and fed into the hard decision Viterbi decoder to recover transmitted video data bits.

\subsection{Mobile Channel Model}

Since the bandwidth of the spread spectrum signal is usually much wider than the coherence bandwidth of the channel, the channel is considered to be frequency-selective, as explained in 
Section 2.4.1. As such, the channel can then be modeled by a tapped delay line with statistically independent time-variant tap weights $\left\{c_{n}(t)\right\}$ as shown in Fig. 4.3 [26].

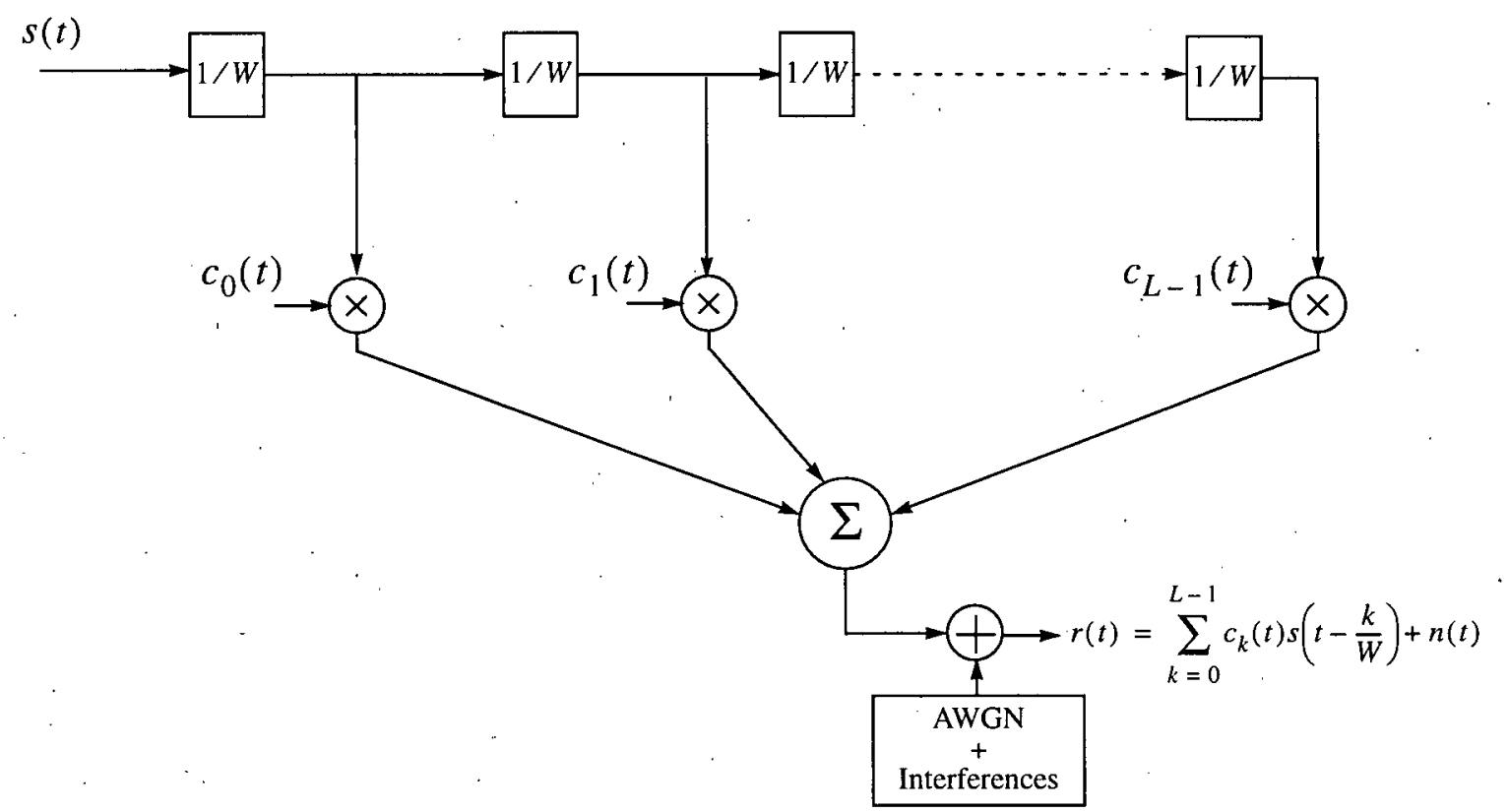

Fig. 4.3 Tapped Delay Line Model of Frequency-Selective Fading Channel [26].

In the figure, $\left\{c_{n}(t) ; n=0,1, \ldots, L-1\right\}$ are independent complex-valued stationary random processes. Their magnitudes $\left|c_{n}(t)\right| \equiv \beta_{n}(t)$ are assumed to be Nakagami- $m$ distributed with PDF given in (2.6). The phases $\angle c_{n}(t)=\left\{\theta_{n}(t)\right\}$ are uniformly distributed over $[0,2 \pi)$ and are independent of $\left\{\beta_{n}(t)\right\}$ [23], [29]. The time delay blocks of $1 / W$ represent the resolution of the multipath delay profile where $W$ is the bandwidth occupied by the real bandpass signal. $n(t)$ is the complex-valued zero-mean white Gaussian noise process with two-sided power spectral density of $\eta_{0} / 2$. The number of multipaths $L$, which may be a random number, is bounded by

$$
\left\lfloor\frac{T_{m}}{T_{c}}\right\rfloor+1
$$

where $\lfloor x\rfloor$ is the floor function, $T_{m}$ is the maximum multipath spread of the channel and $T_{c}$ is 
the chip duration. $T_{m}$ is assumed to be less than the bit interval $T$ such that intersymbol interference (ISI) may be neglected. This is a reasonable assumption for bit-rates of $200 \mathrm{kHz}$ or less [23], due to the fact that the typical delay spread $T_{m}$ for urban areas is typically less than $5 \mu s$ [30]. For instance, considering a system with data bit-rate $1 / T=64 \mathrm{kbits} / \mathrm{s}$ and processing gain $N=64$, the resulting chip duration, $T_{c}=T / N$, is around $25 \mu \mathrm{s}$. Therefore, the maximum number of multipaths $L_{\max }=5$ as given by (4.1).

In cases of scattering processes which generate pure diffusive wave-fields, the fading figure $m \approx 1$ and the Nakagami distribution is identical to the Rayleigh distribution. In the presence of direct component, the Nakagami- $m$ distribution approximates the Rician distribution with $m>1$. The approximate relationship between the Rician $\kappa$ factor and Nakagami $m$ fading figure has been described in (2.8).

\subsection{Correlated Nakagami Fading Simulator}

To simulate the time-selective correlated fading channel of mobile radio environment, a software simulator was implemented based on the physical model for the radio wave propagation process in different scattering environments [51]. This approach deviates from other more conventional simulators for time-varying mobile radio channels, which use digital filters to filter white Gausșian noise to model Doppler spectra [41]. These conventional methods produce Doppler spectrum which only approximates the actual one because the digital filter used in the noise generator must be implementable. However, in the real world, the measured Doppler spectra usually have irregularities that differ themselves from the perfectly smooth U-Tub shape of the spectrum generated by the transfer function [24]. The approach followed here employs a Doppler spectrum generated by a direct summation of the various partial waves, which is an exact replica 
of the actual physical situation.

This superposition of partial waves constitutes the Random Phasor Interference problem in which the total phasor can be interpreted as a vector sum in the complex plane as shown in Fig. 4.4 [51]. When applying the model to the physical situation of radio wave propagation, the total

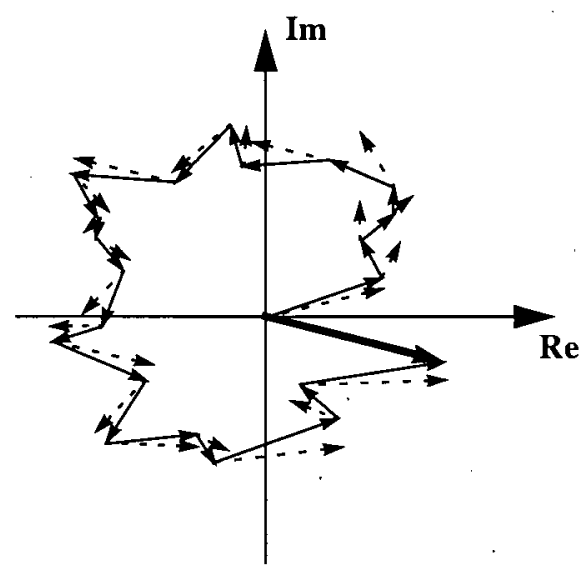

solid: $t=0$

dotted: $t=\Delta t$

bold: total phasor at $t=0$

Fig. 4.4 Example of a Phasor Summation of Partial Waves

phasor (bold-line vector in Fig. 4.4) represents the total wave field of a mobile signal. The total phasor is the vector sum of numerous random phasors (solid-line vectors). In analogy, the total wave field is the summation of a continuum of partial waves caused by scatterings.

To take the Doppler effect into account, consider a short time period $\Delta t$ for which the mobile velocity $\underline{v}$ causes a change of receiver position by $\underline{v} \cdot \Delta t$. Assuming relatively large distances between scatterers and the mobile, we can approximate the phase shifts of the partial waves (dotted-line phasors) caused by the mobile movement as $\frac{2 \pi}{\lambda} \nu \Delta t \cos \alpha_{j}$, where $v=|\underline{v}|$ is the magnitude of $\underline{v}, \lambda$ is the wavelength and $\alpha_{j}$ is the incident angle of the $j$ th partial waves relative to the mobile's velocity vector $\underline{v}$.

In terms of a mathematical expression, the time-dependent total wave field can thus be 
expressed as [37]

$$
E_{Z}(t)=\sum_{j=1}^{\aleph} e_{j} \exp \left\{i\left(\varphi_{j}^{0}+\frac{2 \pi}{\lambda} v t \cos \alpha_{j}\right)\right\}+e_{0} \exp \left\{i\left(\varphi_{0}^{0}+\frac{2 \pi}{\lambda} v t \cos \alpha_{0}\right)\right\}
$$

where $e_{j}$ and $\varphi_{j}^{0}$ are the $j$ th partial wave amplitude and original phase, and $\aleph$ is the total number of scattered partial waves. In the above equation, the first summation term represents the superposition of partial waves, whereas the second term represents a (possibly strong) component resulting from direct or specularly reflected waves. Clearly, the Doppler shifting effect is taken into account by the phase shift factor $\frac{2 \pi}{\lambda} v t \cos \alpha_{j}$ in the phase component terms.

As far as short-term fading is concerned, over a short time interval $\Delta t$, the quantities $e_{j}$, $\varphi_{j}^{0}$ and $\alpha_{j}$ can be assumed to be constant, because the distances between scatterers and receiver are large compared to the mobile's motion in $\Delta t$. Thus, their values are fixed over the mobile run in fading simulation.

For a Nakagami fading channel, the amplitude $r=\left|E_{Z}(t)\right|$ follows the Nakagami-m distribution described in (2.6). Nakagami has shown that the following relations exist between chosen Nakagami parameters and physical partial wave parameters [35]:

$$
\begin{aligned}
& \Omega=N \cdot \overline{e_{j}^{2}}+e_{0}^{2} \\
& m=\frac{\left(N \cdot \overline{e_{j}^{2}}+e_{0}^{2}\right)^{2}}{\left(N \cdot \overline{e_{j}^{2}}+e_{0}^{2}\right)^{2}-e_{0}^{4}}
\end{aligned}
$$

which can be solved to give [51]

$$
\begin{aligned}
e_{0} & =\sqrt{\Omega \sqrt{1-1 / m}} \\
\aleph \cdot \bar{e}_{j}^{2} & =\Omega(1-\sqrt{1-1 / m}) .
\end{aligned}
$$

Thus, for a certain fading characteristics quantified by the average power $\Omega$ and the fading 
figure $m$, the partial wave parameters, $\aleph, e_{0}$ and $\left\{e_{j} ; j=1,2, \ldots, \aleph\right\}$ can be determined. The authors in [51] suggest around 100 partial waves to be sufficient to reproduce the actual physical situation.

The following graphs show the comparison between the simulated signal and the actual Nakagami distribution with different values of fading figures $m$. The left side of Fig. 4.5 and Fig. 4.6 show the histograms of the Nakagami fading simulator output using $1 \times 10^{6}$ data samples with
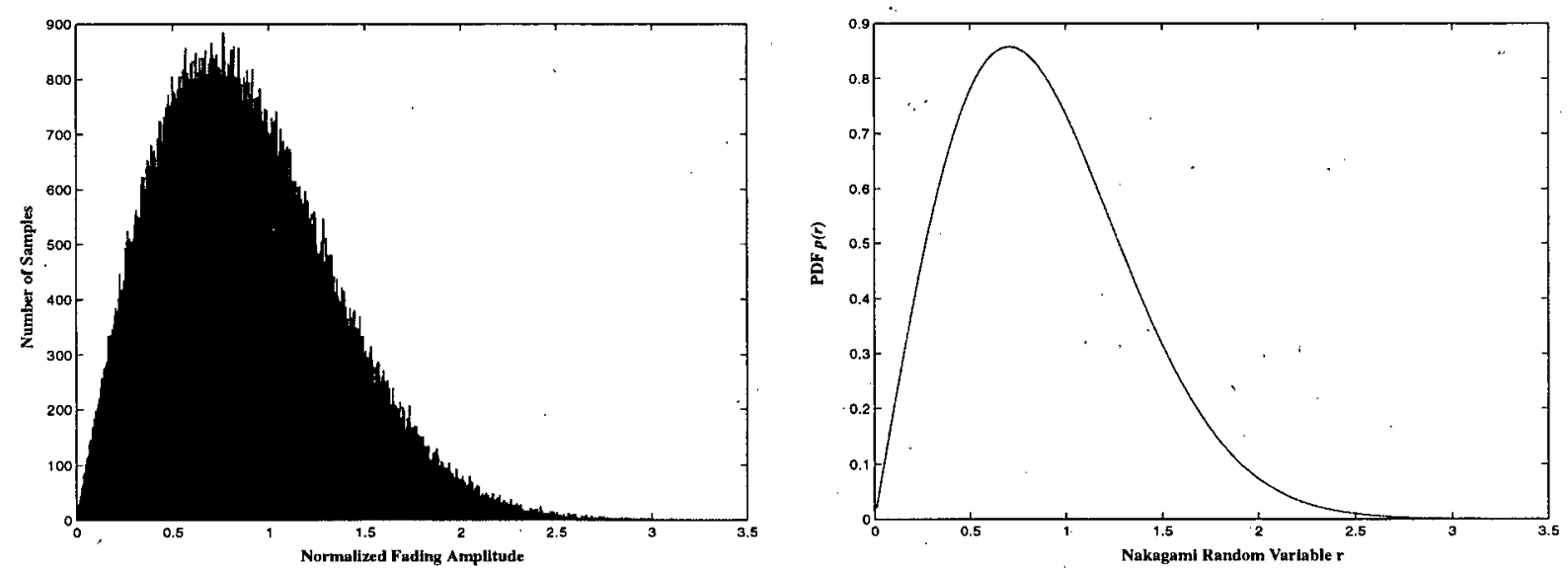

Fig. 4.5 Comparison of Nakagami Fading Simulator and PDF for $m=1$
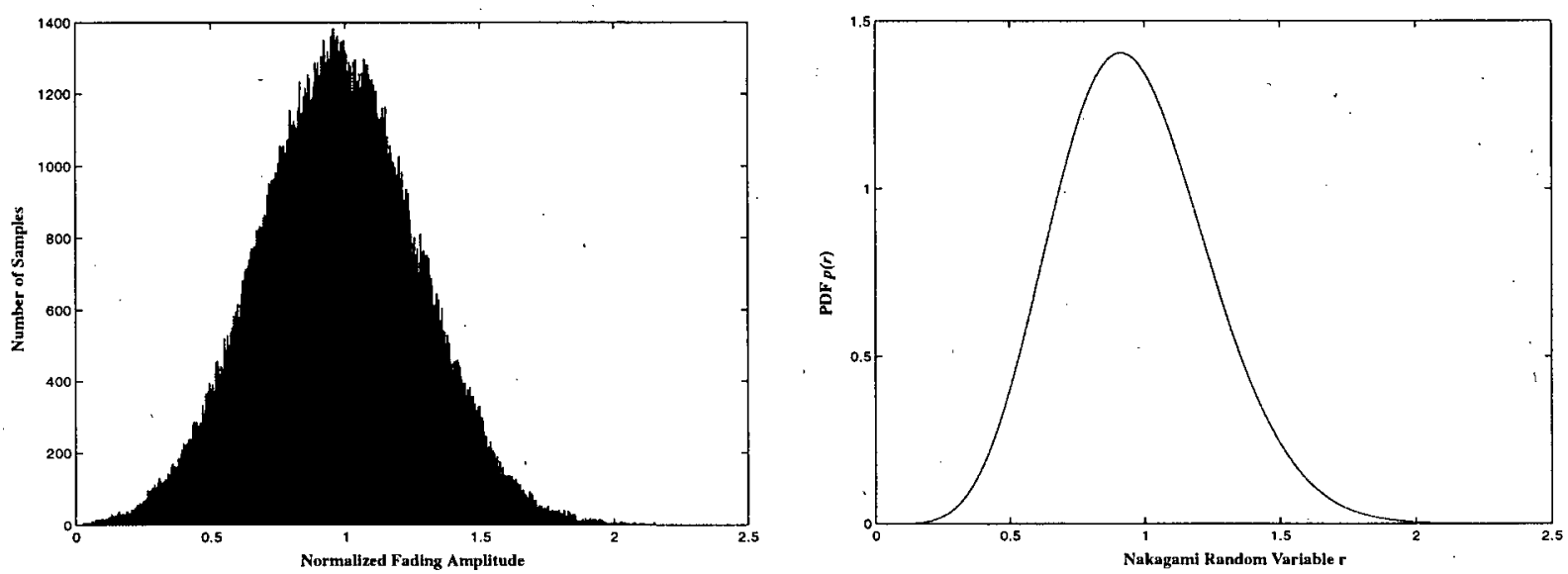

Fig. 4.6 Comparison of Nakagami Fading Simulator and PDF at for $m=3$ 
fading figure $m=1$ and 3, respectively. In both cases, the average power $\Omega$ is set to be unity, total number of partial waves $\mathrm{N}=100$, and velocity $v=27.78 \mathrm{~m} / \mathrm{s}$, which is equivalent to 100 $\mathrm{km} / \mathrm{hr}$. The right side of the figures are the PDF plots of Nakagami $-m$ distribution of corresponding fading figures for comparison. As shown in both figures, the fading simulator produces samples with excellent accuracy with respect to the theoretical distributions. To measure the accuracy of the simulator outputs quantitatively, we compare the Cumulative Probability Function (CDF) of the Nakagami- $m$ distribution with the number of sample counts in the histogram. As an example, from the simulator output histogram of Fig. 4.5, we obtain a minor deviation of $0.91 \%$ from the theoretical $\mathrm{CDF}$ value at $r=0.5$. Thus, the implemented fading simulator produces accurate fading outputs following Nakagami- $m$ distribution.

\subsection{Receiver Structure}

To take advantage of the wideband characteristics of spread spectrum signals, a coherent RAKE receiver is employed in the system to provide multipath diversity. To simplify the mathematical analysis, we will be assuming that perfect knowledge of the channel phase and gain can be obtained [23], [29]. However, for computer simulation, perfect knowledge of channel amplitude is not required. Coherent RAKE combining method weights the resolved multipaths in proportion to their instantaneous received signal envelopes and adds the components constructively.

Fig. 4.7 shows the receiver structure for reference user $(k=0)$, where the number of fingers, $L_{r}$, is a variable parameter less than or equal to the total number of multipaths $L$, as defined in (4.1). $L_{r}$ is made to be a variable so that the effect of diversity may be observed. The matched filter is matched to the reference user's CDMA spreading code and is assumed to have achieved time synchronization with the initial path of the reference signal. The tap weights 


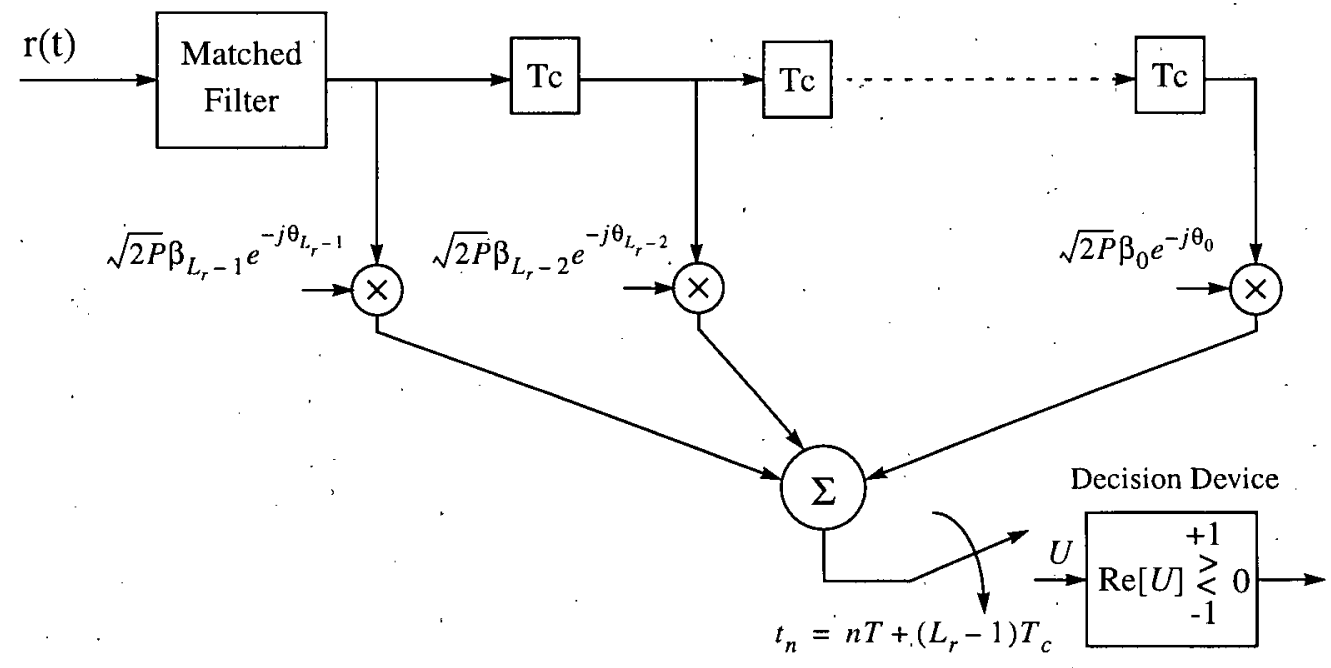

Fig. 4.7 Coherent RAKE Receiver Model

$\left\{\beta_{i} ; i=0, \ldots, L_{r}-1\right\}$ and phases $\left\{\theta_{i} ; i=0, \ldots, L_{r}-1\right\}$ are assumed to be the perfect estimates of the channel parameters. In practice, the estimation and coherent demodulation may be done jointly through pilot tone calibration techniques [27], [28]. The sampling times of the receiver are $t_{n}=n T+\left(L_{r}-1\right) T_{c}$, where $n$ is an integer index. The first term is from the matched filter sampling time, and the second term is from the combining of the $\left(L_{r}-1\right)$ paths following the initial path. The sampling output $U$ is then used in the decision device for demodulation decision.

\subsection{CDMA Forward Link BER Performance Analysis}

In this section, we will derive mathematical expressions for the signals in a single-cell CDMA forward link system. In such a system, the transmitted signal for the $k$ th user is a phase-coded carrier expressed as

$$
s^{(k)}(t)=\sqrt{2 P} a^{(k)}(t) b^{(k)}(t) \cos \left(\omega_{c} t+\phi\right)
$$


where $a^{(n)}(t)$ is the spreading code sequence of the $k$ th user,

$$
a^{(k)}(t)=\sum_{j=-\infty}^{\infty} a_{j}^{(k)} p_{a}\left(t-j T_{c}\right), \quad a_{j}^{(k)} \in\{-1,1\}
$$

and $b^{(k)}(t)$ is the data waveform,

$$
b^{(k)}(t)=\sum_{j=-\infty}^{\infty} b_{j}^{(k)} p_{b}(t-j T), \quad b_{j}^{(k)} \in\{-1,1\}
$$

In (4.4), $P=E_{b} / T$ is the average transmitted power assumed to be equal for all users, where $E_{b}$ is the signal energy per bit and $T$ is the bit duration. $\omega_{c}$ is the common carrier radian frequency, and $\phi$ is the initial phase of the modulator uniformly distributed around $[0,2 \pi)$. In (4.5) and (4.6), $T_{c}$ is the chip duration, $p_{a}(t)$ and $p_{b}(t)$ are rectangular pulses of unit height and duration of $T_{c}$ and $T$, respectively.

For synchronous operation with $K$ simultaneous users, the composite signal at the input of the channel of the CDMA forward link is

$$
s(t)=\sum_{k=0}^{K-1} \sqrt{2 P} a^{(k)}(t) b^{(k)}(t) \cos \left(\omega_{c} t+\phi\right)
$$

For a single-cell, multi-user model, the received signal after the channel is

$$
r(t)=\sqrt{2 P} \sum_{k=0}^{K-1} \sum_{l=0}^{L-1} \beta_{l} \cdot a^{(k)}\left(t-\tau_{l}\right) \cdot b^{(k)}\left(t-\tau_{l}\right) \cos \left(\omega_{c} t+\varphi_{l}\right)+n(t)
$$

where $K$ is the total number of users in the cell, and $L$ is the number of multipaths of random value upper bounded by (4.1). $\varphi_{l}=\phi+\theta_{l}-\omega_{c} \tau_{l}$ is the phase of the $l$ th path, where $\theta_{l}$ is the channel phase shift and $\tau_{l}$ is the multipath time delay for the $l$ th path. Each path is assumed to fade independently with fading coefficient $\beta_{l} e^{j \theta_{l}}$ of which the amplitude $\beta_{l}$ follows a Nakagami-m 
distribution and the phase $\theta_{l}$ follows an uniform distribution over $[0,2 \pi)$. Finally, $n(t)$ is the additive white Gaussian noise process.

Each signal path which matches with the $n$th RAKE finger gives a desired signal component denoted as $S^{(n)}$. In addition, at the $n$th finger of the RAKE receiver, there is multipleaccess interference denoted as $I_{m a i}^{(n)}$ generated from the cross-correlation between the reference user's code and other users' codes. Moreover, there is self-noise interference denoted as $I_{s i}^{(n)}$ produced by cross-correlation with multipath delay copies of its own signal. Finally, AWGN contributes noise interference term denoted as $I_{n i}^{(n)}$ to the desired signal. In [29], the coherent RAKE receiver output is given for an asynchronous (chip-misaligned) CDMA system. To adapt the receiver output for a synchronous system as in the case of forward link, we replace the independent fading amplitudes and signal phases for each user's multipath signal by common values which are shared by all users in the same cell. Thus, the response of the coherent RAKE receiver with $L_{r}$ fingers at each sampling time $t_{n}=n T+\left(L_{r}-1\right) T_{c}$ can be expressed as

$$
\begin{aligned}
U & =\sum_{n=0}^{L_{r}-1\left(T+n T_{c}\right)} \int_{n T_{c}} r(t) \beta_{n} a^{(1)}\left(t-n T_{c}\right) \cos \left(\omega_{c} t+\varphi_{n}\right) d t \\
& =\sum_{n=0}^{L_{r}-1}\left\{S^{(n)}+I_{m a i}^{(n)}+I_{s i}^{(n)}+I_{n i}^{(n)}\right\}
\end{aligned}
$$

where the four output response components are given by:

$$
\begin{aligned}
& S^{(n)}=\sqrt{\frac{P}{2}} \cdot b_{0}^{(0)} T \cdot\left\{\beta_{n}\right\}^{2} \\
& I_{\text {mai }}^{(n)}=\sqrt{\frac{P}{2}} \cdot \sum_{k=1}^{K-1 L^{(k)}-1} \sum_{l=0} \beta_{n} \beta_{l} \cdot\left\{b_{-1}^{(k)} R_{k 1}\left[\tau_{n l}\right]+b_{0}^{(k)} \hat{R}_{k 1}\left[\tau_{n l}\right]\right\} \cdot \cos \left(\varphi_{n l}\right)
\end{aligned}
$$




$$
\begin{aligned}
& I_{s i}^{(n)}=\sqrt{\frac{P}{2}} \cdot \sum_{\substack{l=0 \\
l \neq n}}^{L^{(0)}-1} \beta_{n} \beta_{l} \cdot\left\{b_{-1}^{(k)} R_{00}\left[\tau_{n l}\right]+b_{0}^{(0)} \hat{R}_{00}\left[\tau_{n l}\right]\right\} \cdot \cos \left(\varphi_{n l}\right) \\
& I_{n i}^{(n)}=\int_{n T_{c}}^{T+n T_{c}} n(t) \beta_{n} a^{(0)}\left(t-n T_{c}\right) \cos \left(\omega_{c} t+\varphi_{n}\right) d t
\end{aligned}
$$

In the above equations, $b_{0}^{(0)}$ is the information bit of the reference user to be detected whereas $b_{-1}^{(0)}$ is the preceding bit. $\tau_{n l}=\tau_{l}-\tau_{n}$ is the time delay difference and $\varphi_{n l}=\varphi_{l}-\varphi_{n}$ is the phase difference between the $l$ th and the $n$th multipath, respectively. $R_{k 0}(\tau)$ and $\hat{R}_{k 0}(\tau)$ are the continuous-time partial cross-correlation functions between the $k$ th and the reference user and are defined by [31] as

$$
\begin{aligned}
R_{k 0}(\tau) & =\int_{0}^{\tau} a_{k}(t-\tau) a_{0}(t) d t \\
\hat{R}_{k 0}(\tau) & =\int_{\tau}^{T} a_{k}(t-\tau) a_{0}(t) d t .
\end{aligned}
$$

We need to transform the continuous-time partial cross-correlation functions into discrete form to evaluate for discrete binary sequences. By choosing $l$ to fulfill $0 \leq l T_{c} \leq \tau \leq(l+1) T_{c} \leq T$, then these two cross-correlation functions can be written as [31]

$$
\begin{aligned}
& R_{k 0}(\tau)=C_{k 0}(l-N) T_{c}+\left[C_{k 0}(l+1-N)-C_{k 0}(l-N)\right] \cdot\left(\tau-l T_{c}\right) \\
& \hat{R}_{k 0}(\tau)=C_{k 0}(l) T_{c}+\left[C_{k 0}(l+1)-C_{k 0}(l)\right] \cdot\left(\tau-l T_{c}\right)
\end{aligned}
$$

where $C_{k 0}$ is the discrete aperiodic cross-correlation function for the sequences $\left\{a_{j}^{(k)}\right\}$ and $\left\{a_{j}^{(0)}\right\}$ defined as 


$$
C_{k 0}(l)=\left\{\begin{array}{cl}
\sum_{j=0}^{N-1-l} a_{j}^{(k)} a_{j+l}^{(i)} & 0 \leq l \leq N-1 \\
\sum_{j=0}^{N-1+l} a_{j-l}^{(k)} a_{j}^{(i)} & 1-N \leq l<0 \\
0 & |l| \geq N .
\end{array}\right.
$$

For a chip-synchronized system which is considered in this thesis, the residual cross-correlation values represented by the second term of (4.12) and (4.13) are zero. Thus, we can simplify the partial cross-correlation functions as follows:

$$
\begin{aligned}
& R_{k 0}(\tau)=C_{k 0}(l-N) T_{c} \\
& \hat{R}_{k 0}(\tau)=C_{k 0}(l) T_{c}
\end{aligned}
$$

\subsection{Forward Link BER Analysis with the Gaussian Approximation}

The RAKE receiver output $U$ in (4.9) is the summation of the output of each finger, which consists of the desired signal component, multiple-access interference, self-noise interference and AWGN components. In order to simplify the BER performance analysis of CDMA systems, the Gaussian approximation is often applied to the interference terms [5], [23], [29], [33]. It is found to be quite accurate even for small values of $K$ (e.g. less than 10 ) when $\mathrm{BER} \geq 10^{-3}$. (see for example [42]). Therefore, in this section, we will apply Gaussian approximation on the multipleaccess interference $I_{m a i}^{(n)}$ and the self-noise interference $I_{s i}^{(n)}$ in (4.9b) and (4.9c) for BER performance analysis of the CDMA forward link.

The variance of multiple-access interference $\sigma_{M A I, n}^{2}$ for an asynchronized CDMA system at the $n$th finger of the receiver, conditioned on $\beta_{n}$, can be expressed as [29] 


$$
\begin{aligned}
\sigma_{M A I, n}^{2}= & \frac{P}{2} \cdot \sum_{k=1}^{K-1} \sum_{l=0}^{L-1}\left\{\beta_{n}\right\}^{2} E\left[\left\{\beta_{l}\right\}^{2}\right] \\
& E\left[\left\{b_{-1}^{(k)} R_{k 0}\left(\tau_{n l}\right)+b_{0}^{(k)} R_{k 0}\left(\tau_{n l}\right)\right\}^{2}\right] \cdot E\left[\cos ^{2}\left(\varphi_{n l}\right)\right] \\
= & \frac{P}{4}\left\{\beta_{n}\right\}^{2} \sum_{k=1}^{K-1} \frac{T^{2}}{3 N^{3}} r_{K}(N) \sum_{l=0}^{L-1} E\left[\left\{\beta_{l}\right\}^{2}\right] .
\end{aligned}
$$

The detailed derivation of (4.17) is presented in Appendix A. $r_{K}(N)$ is the average cross-correlation parameter over all the possible $\left(\begin{array}{l}K \\ 2\end{array}\right)=\{K(K-1) / 2\}$ combinations of sequence pairs among $K$ spreading sequences with length of $N$ [31]. For the purpose of the CDMA BER performance evaluation, we will be using random binary spreading sequences for both the analysis and Monte Carlo simulations. It is equivalent to the assumption that code sequences of period much longer than the processing gain is being used. The resulting value of $r_{K}(N)$ for random binary spreading sequences is found to be $2 N^{2}$ in [29] which is also confirmed by our simulation results. The data bits $b_{-1}^{(k)}$ and $b_{0}^{(k)}$ in (4.17) are considered to be identically, independent distributed (i.i.d.) random variables with equal probability of taking values +1 or -1 .

For the CDMA forward link system that we are considering, chip synchronization among signals can be assumed. Thus, to obtain the conditional variance of multiple-access interference for a synchronous system, we follow a similar approach as in [23] and [29] by adding a multiplication factor of $3 / 2$ to (4.17). This correction factor is used to compensate for the increased interference statistics from overlapping chips among the reference user and other users due to chip synchronization. The compensation is further justified by the results obtained by Fong et al. in [33]. By substituting the value of $r_{K}(N)$ and adding the chip-synchronized compensation factor to (4.17), the conditional variance of the multiple-access interference $\sigma_{M A I, n}^{2}$ can now be simplified to 


$$
\sigma_{M A I, n}^{2}=\frac{P T^{2}}{4 N}\left\{\beta_{n}\right\}^{2} \cdot \sum_{k=1}^{K-1} \sum_{l=0}^{L-1} \Omega_{l}
$$

where $\Omega_{l}=E\left[\left\{\beta_{l}\right\}^{2}\right]$ is the average power for the signal of the $l$ th path.

Self-noise interference $\sigma_{S I, n}^{2}$ can be derived using the similar approach as in [23]. Selfnoise (SI) may be considered as an additional multiple-access user where instead of $L$ paths, there would only be $L-1$ paths at the input to the receiver because one path contributes to the desired signal component $U_{s}$. Thus, self-noise interference can be approximated by

$$
\sigma_{S I, n}^{2} \cong \frac{P T^{2}}{4 N} \cdot\left\{\beta_{n}\right\}^{2} \sum_{l=1}^{L-1} \Omega_{l}
$$

From (4.9d), the variance of AWGN conditioned on $\beta_{n}$ is [29]

$$
\sigma_{N I, n}^{2}=\frac{T \eta_{o}}{4} \cdot\left\{\beta_{n}\right\}^{2}
$$

Therefore, the response of the reference receiver $U$ to the received signal at any sampling instant can now be modeled as a conditional complex Gaussian random variable with conditional mean of the desired signal component of the total received signal,

$$
U_{s}=\sqrt{\frac{E_{b} T}{2}} \cdot \sum_{n=0}^{L_{r}-1}\left\{\beta_{n}\right\}^{2}
$$

and conditional variance equals to the sum of all interference terms, 


$$
\begin{aligned}
\sigma_{S}^{2} & =\sum_{n=0}^{L_{r}-1}\left(\sigma_{M A I, \mathrm{n}}^{2}+\sigma_{S I, n}^{2}+\sigma_{N I, n}^{2}\right) \\
& =\left(\frac{E_{b} T}{4}\right) \cdot\left\{\frac{\sum_{k=1}^{K-1} \sum_{l=0}^{L-1} \Omega_{l} \sum_{l=1}^{L-1} \Omega_{l}}{N}+\frac{\eta_{o}}{E_{b}}\right\} \cdot \sum_{n=0}^{L_{r}-1}\left\{\beta_{n}\right\}^{2} .
\end{aligned}
$$

An exponential multipath intensity profile (MIP) is assumed for the average power $\Omega_{l}$ at the output of the channel as a function of path delay. The assumption is justified by the actual measurements made by Turin in an urban environment [38]. Therefore, we have

$$
\Omega_{l}=\Omega_{0} e^{-l \delta}, \quad \delta \geq 0
$$

where $\Omega_{0}$ is the first path average signal strength and $\delta$ is the rate of average power decay. Substituting (4.23) into (4.22) yields

$$
\sigma_{S}^{2}=\left(\frac{E_{b} T \Omega_{0}}{4}\right) \cdot\left\{\frac{(K-1) q(L, \delta)}{N}+\frac{q(L, \delta)-1}{N}+\frac{\eta_{o}}{E_{b} \Omega_{0}}\right\} \cdot \sum_{n=0}^{L_{r}-1^{\prime}}\left\{\beta_{n}\right\}^{2}
$$

where $q(L, \delta) \equiv \sum_{l=0}^{L-1} e^{-l \delta}=\left(1-e^{L \delta}\right) /\left(1-e^{-\delta}\right)$

The received signal-to-noise ratio (SNR) at the output of the receiver is

$$
\operatorname{SNR}=\frac{U_{s}^{2}}{\sigma_{s}^{2}}
$$

By denoting the random component of the SNR as

$$
S=\frac{1}{\Omega_{0}} \cdot \sum_{n=0}^{L_{r}-1}\left\{\beta_{n}\right\}^{2}
$$

and the deterministic component as 


$$
\Upsilon=\left\{\frac{1}{2} \cdot\left(\frac{(K-1) q(L, \delta)}{N}+\frac{q(L, \delta)-1}{N}+\frac{\eta_{o}}{E_{b} \Omega_{0}}\right)\right\}^{-1}
$$

the received SNR can be written in compact form as $\Upsilon S$.

We observe that when there is an absence of multipath (i.e. $L=1$ ), then $q(L, \delta)=1$. Thus, the self-noise term represented by $\frac{q(L, \delta)-1}{N}$ in (4.27) becomes zero. The self-noise interference vanishes in a single path environment because it originates from the cross-correlation of the reference user in the existence of different multipath signals.

For coherent demodulation in the presence of AWGN, the probability of error conditioned on the instantaneous signal-to-noise ratio (SNR) can be expressed as [23]

$$
P_{e}(S)=Q(\sqrt{\Upsilon S})
$$

where $Q(x)=\frac{1}{\sqrt{2 \pi}} \int_{x}^{\infty} e^{-x^{2} / 2} d x$. To obtain the average error probability for random variable $S$, the average of $P_{e}(S)$ over the PDF of $S$ has to be obtained as follows:

$$
\bar{P}_{e}=\int_{0}^{\infty} P_{e}(S) p(S) d S
$$

The solution for the average error probability $\overline{P_{e}}$ for the general case of non-integer Nakagami fading has been evaluated in [23] as

$$
\overline{P_{e}}=\sqrt{\frac{\Upsilon_{s}}{1+\Upsilon_{s}}} \cdot \frac{\left(1+\Upsilon_{s}\right)^{-m_{s}} \Gamma\left(m_{s}+\frac{1}{2}\right)}{2 \sqrt{\pi} \Gamma\left(m_{s}+1\right)} \cdot{ }_{2} F_{1}\left(1, m_{s}+\frac{1}{2}, m_{s}+1, \frac{1}{1+\Upsilon_{s}}\right)
$$

where

$$
m_{s}=\left(\sum_{i=0}^{L_{r}-1} e^{-\delta i}\right)^{2} /\left(\sum_{i=0}^{L_{r}-1} \frac{\left(e^{-\delta i}\right)^{2}}{m}\right)=m \frac{q\left(L_{r}, \delta\right)^{2}}{q\left(L_{r}, 2 \delta\right)}
$$




$$
\Omega_{s}=\sum_{i=0}^{L_{r}} e^{-\delta(i-1)}=q\left(L_{r}, \delta\right)
$$

and

$$
\Upsilon_{s}=\frac{\Upsilon \Omega_{s}}{2 m_{s}}=\frac{\Upsilon q\left(L_{r}, 2 \delta\right)}{2 m q\left(L_{r}, \delta\right)}
$$

In the above equations, $\Gamma(\cdot)$ is the Gamma function defined as $\Gamma(x)=\int_{0}^{\infty} e^{-t} t^{x-1} d t, \quad x>0$ and ${ }_{2} F_{1}(\cdot)$ is the hypergeometric function defined as ${ }_{2} F_{1}(a, b, c, d)=\sum_{k=0}^{\infty} \frac{(a)_{k}(b)_{k} d^{k}}{(c)_{k} k !}$ with $(a)_{k}=a(a+1) \cdots(a+k-1), \quad(a)_{0}=1$. The analytical BER performance of the CDMA forward link is evaluated through numerical computations of (4.30)-(4.30c). The results will then be compared to those obtained from the Monte Carlo simulation method.

\subsection{Computer Simulation System Description}

Besides evaluating the CDMA forward link BER performance through analysis, we also compare the results with those obtained from Monte Carlo computer simulation techniques based on the response of the coherent RAKE receiver given in (4.9). During the simulations, the $k$ th user $(k=0, \ldots, K-1)$ is assigned a random signature sequence $\left\{a^{(k)}(i) ; i=1, \ldots, N-1\right\}$ of processing gain $N$. For each simulation run, the preceding information bit $b_{-1}^{(k)}$, and the information bit to be detected $b_{0}^{(k)}$, are assigned to the $k$ th user. $b_{-1}^{(k)}$ and $b_{0}^{(k)}$ take values from the alphabet $\{ \pm 1\}$ with equal probabilities and are i.i.d. The fading signal amplitude of the $n$th multipath $\beta_{n}$, is a random variable with Nakagami- $m$ distribution, and is generated by means of computer simulation using the Nakagami fading simulator as described in Section 4.4. The $l$ th multipath delay $\tau_{l}$ is randomly generated in discrete units of the chip duration $T_{c}, 0 \leq \tau_{l}<T$. In addition, the phase of the $n$th path of the carrier $\varphi_{n}$ is a random variable with a uniformly distri- 
bution over $[0,2 \pi)$. Lastly, AWGN term $n(t)$ is generated by a Gaussian random process with zero mean and variance given by (4.20).

At the $n$th finger of the coherent RAKE receiver, the desired signal component $S^{(n)}$ along with other interference components such as multiple-access interference $I_{m a i}^{(n)}$, self-noise interference $I_{s i}^{(n)}$ and noise interference term $I_{n i}^{(n)}$, are generated according to (4.9a)-(4.9d). These components are summed up to form the output of each finger. The total output from $L_{r}$ number of fingers of the reference user's RAKE receiver forms the decision output $U$. shown in Fig. 4.7. The detected bit is then compared with the transmitted bit to check if an error is made. The process is repeated to receive an adequate number of errors (e.g. greater than 500) in order to achieve a $95 \%$ confidence interval of $\pm 5 \%$ of the average BER.

\subsection{BER Performance Evaluation Results for CDMA Forward Link}

In this section, we present the numerical results for the BER performance of the proposed singlecell CDMA forward link system. Fig. 4.8 compares the BER performance of the CDMA forward link obtained from both analytical method and Monte Carlo simulation techniques. For comparison purposes, similar to the analysis presented in Section 4.7, random spreading sequences are used in Monte Carlo simulations. The transmitted bit energy-to-noise density ratio was selected to have a relatively large value of $E_{b} / \eta_{0}=30 \mathrm{~dB}$ so that AWGN effect is relatively low as compared to the user interferences. Therefore, we can concentrate on the BER performance results of the user-interference-limited CDMA systems in terms of the number of users $K$, while ignoring the insignificant interference from AWGN [33]. The total number of multipaths is set to $L=5$ as previously explained in Section 4.4 , and the cases of using RAKE fingers $L_{r}=2$ and 3 are considered. The processing gain is assumed to be $N=64$ as per IS-95 standard. We use the 
fading figure $m=1$ for the Nakagami fading channel which is equivalent to Rayleigh fading commonly encountered in urban environments [5].

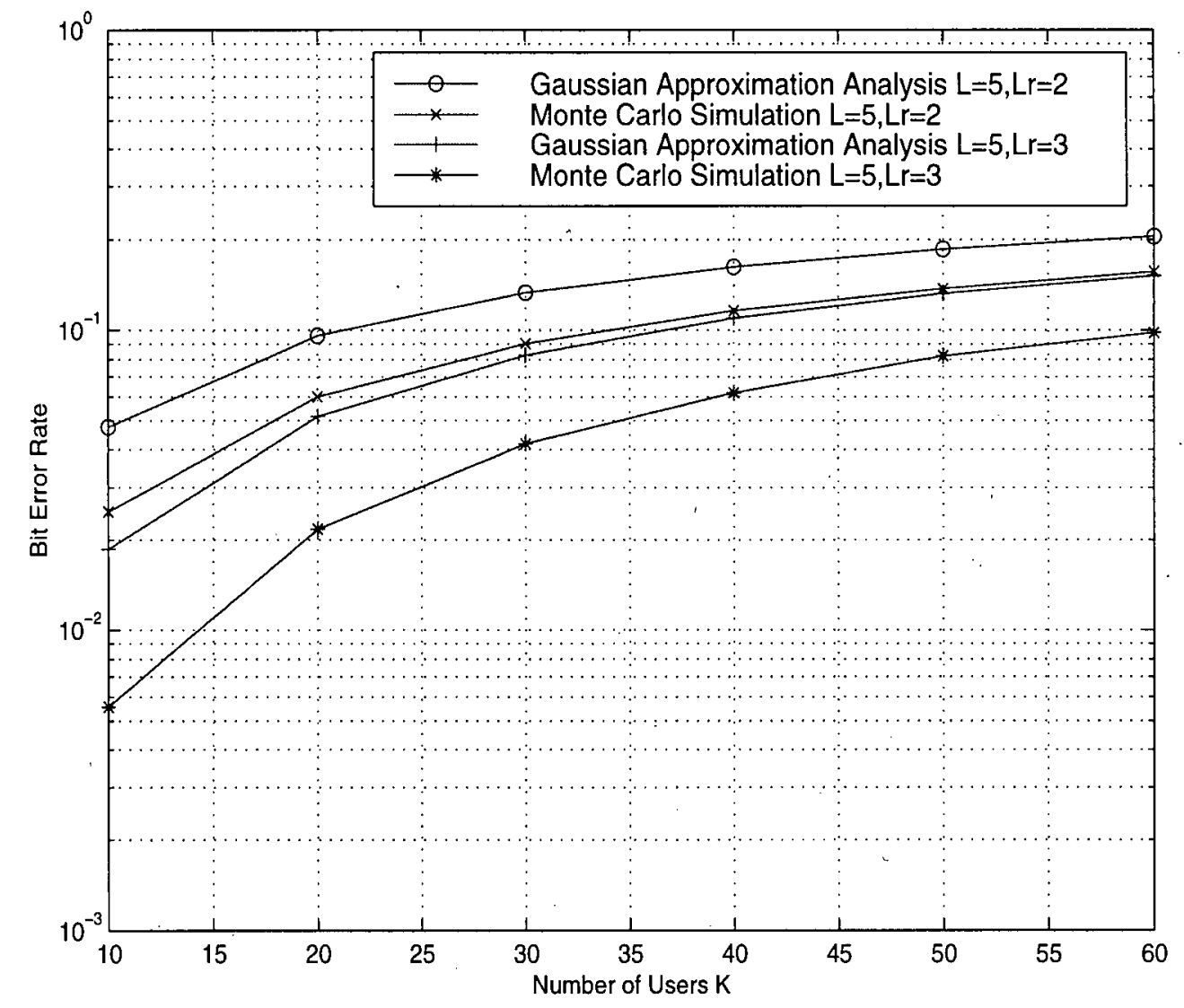

Fig. 4.8 Single-Cell CDMA Forward Link BER Performance

As shown in the figure, the BER performance results obtained from the analysis using the Gaussian approximation are very optimistic with respect to the simulation results for both cases of $L_{r}=2$ and 3 . The BER performance differences can be explained by the fact that the Gaussian approximation used in the analysis is based on the central limit theorem, which assumes the random variables to be i.i.d [26]. During the analysis in Section 4.7, we apply the Gaussian approximation on the multiple-access and self-noise interference terms. However, on a forward 
link the interference signals of other users in the same cell arrive at the reference user with the same channel characteristics such as amplitude fluctuations, phase distortions and transmission time delays. Therefore, the Gaussian approximation does not quite accurately predict the BER performance due to the lack of statistical independency among multiple-access interference signals. However, as we will see in the following chapter, the Gaussian approximation provides much more accurate BER performance predictions for multiple-cell systems.

From the same figure, we also observe that in the case of $L_{\dot{r}}=2$, there is a closer match in BER performance results obtained from the analytical and simulation methods when compared to the case of $L_{r}=3$. Moreover, the result discrepancies between the two methods become smaller as the number of users increases for both cases of $L_{r}$. These observations can be explained by the fact that, in general, the Gaussian approximation provides more accurate results at higher bit error rates [43].

Next, in Fig. 4.9 we compare the BER performance of the proposed CDMA system utilizing concatenated codes and one that uses non-concatenated codes. The purpose is to illustrate the effectiveness of orthogonal spreading in a multipath and multiuser environment. Concatenated coding scheme uses spreading codes generated from modulo- 2 addition between the PN sequence and the Walsh codes. On the other hand, non-concatenated scheme uses spreading codes generated from the maximal-length sequence directly, without performing modulo-2 addition with the Walsh codes. Two cases with different number of multipaths and RAKE fingers are considered here. In both cases, multipath signal of identical and normalized path strengths (i.e. $\Omega_{l}=\Omega=1$ ) are generated with the same fading figure of $m=1$. Spreading gain is $N=64$, the period of the PN sequence is $2^{15}-1$, and $E_{b} / \eta_{0}=10 \mathrm{~dB}$.

The first pair of curves (solid lines) represents the BER performance of non-concatenated 


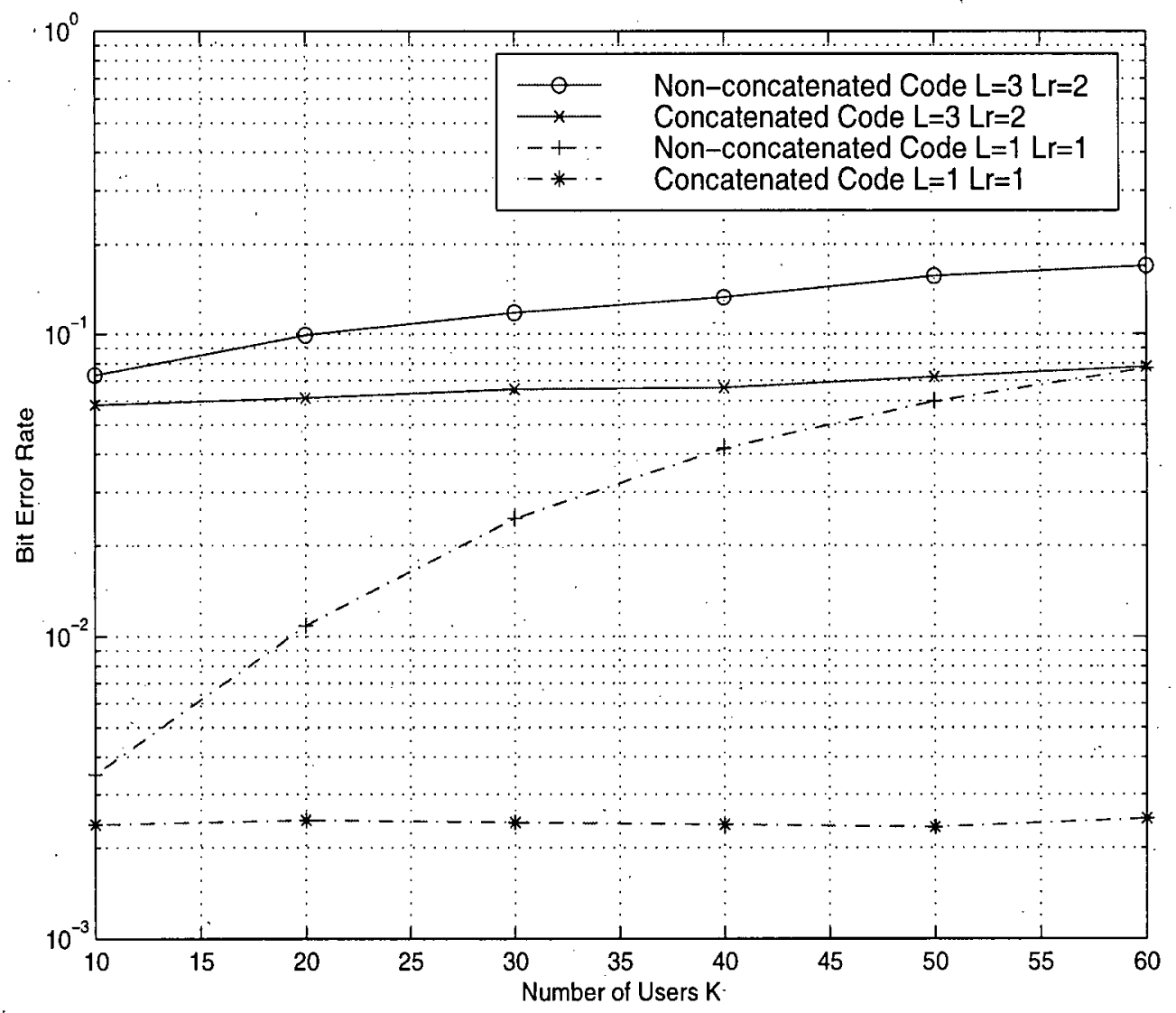

Fig. 4.9 Simulation Results Comparison of Concatenated and Non-Concatenated Schemes codes versus concatenated codes of a CDMA system with $L=3$ and RAKE receiver of $L_{r}=2$. As shown, the concatenated coding scheme provides better performance, and the improvement becomes greater as the number of users increases. This improvement happens because of the orthogonal spreading effect provided by the Walsh codes among users. As the number of users increases, multiple-access interference remains suppressed relative to the non-concatenated coding scheme. In the second set of curves (dotted lines), we assume $L=1$ (i.e., no multipath) instead, and a single correlator receiver is used. We can see a dramatic improvement of the concatenated coding scheme over the non-concatenated one. This is because in the absence of 
multipaths, perfect orthogonality between multiple users' spreading codes is maintained, thus resulting in zero multiple-access interference and self-noise. In fact, the BER performance of the concatenated coding scheme remains constant over the number of users in a single path environment, as the only source of interference is from the AWGN. On the other hand, PN sequences used in the non-concatenated case do not possess such properties and thus, when the number of users increases, multiple-access interference increases accordingly, which results in degradation of BER performance.

\subsection{Video Transmission Performance in CDMA Forward Link}

In this section, we present the numerical results from computer simulation of the proposed video transmission system over a single-cell CDMA forward link operating in correlated Nakagami fading channel environment. We have incorporated the modified H.263 video codec into previously described CDMA system and have evaluated the video performance as a function of PSNR for different number of resolving paths and signal propagation characteristics, as well as for the presence of channel estimation errors.

For the simulations, we use the well-known video sequence Miss America of 100 frames coded by the modified H.263 encoder at a fixed bit-rate of $64 \mathrm{kbits} / \mathrm{s}$. The video sequence represents a typical videophone/video-conferencing situation. It shows the upper half of a woman talking in front of a plain, steady background with natural facial, head and shoulder movements during the conversation. The $64 \mathrm{kbit} / \mathrm{s}$ video data is half-rate convolutionally coded and then spread by the concatenated codes of length 64 , resulting in a chip rate of $8.192 \mathrm{MChip} / \mathrm{s}$. The PN sequence used is a maximal-length sequence of generator polynomial 100003 in octal representation [57], and has a period of $2^{15}-1$ chips. The shift registers are initialized by one 1 and all 
zeros. The 64 Walsh sequences are randomly assigned to each user, except for the all-zero Walsh code which is reserved for the pilot channel.

The frequency-selective channel is simulated by a tapped delay line model as described in Section 4.3 , whereas each tap weight is independently generated by the correlated Nakagami fading simulator as described in Section 4.4. The multipath intensity profile is assumed to be logarithmic with the exponent value made variable to allow for more general case evaluation. Signal phases are random variables uniformly distributed in the interval $[0,2 \pi)$. We assume that the base station continuously transmits a pilot signal which is used by the mobile receiver to make an estimate of the channel impulse response for maximal-ratio combining of different multipath components as well as acquiring synchronization for coherent demodulation. PSNR of each data point is obtained by averaging 20 simulation runs using different random seeds.

Unless stated otherwise, the default values of the system parameters used to obtain the following numerical results are as follows:

- Total number of multipaths $L=3$. This value is chosen to be less than the maximum number of multipaths $L_{\max }=5$ as discussed in Section 4.3 for the assumed maximum multipath spread of $5 \mu s$.

- Number of multipath resolving RAKE fingers $L_{r}=2$.

- Nakagami fading figure $m=1$ for simulating mobile channels in typical urban environment.

- Mobile velocity is $100 \mathrm{~km} / \mathrm{hr}$ and carrier frequency is $2 \mathrm{GHz}$, which results in a maximum Doppler frequency $B_{d}$ of about $180 \mathrm{~Hz}$. Thus, the default channel has $B_{d} T \approx 2.8 \times 10^{-3}$ representing fast fading characteristics. 
- Multipath intensity profile (MIP) is logarithmic with default exponent $\dot{\delta}=0$, which is equivalent to uniform MIP.

In Fig. 4.10, we present the video performance of the modified codec operating in a single-cell CDMA system. We observe a similar PSNR improvement of the modified version over the original version as in Fig. 3.2. In this case, there is 1-4dB PSNR performance improvement over the range of user number under consideration.

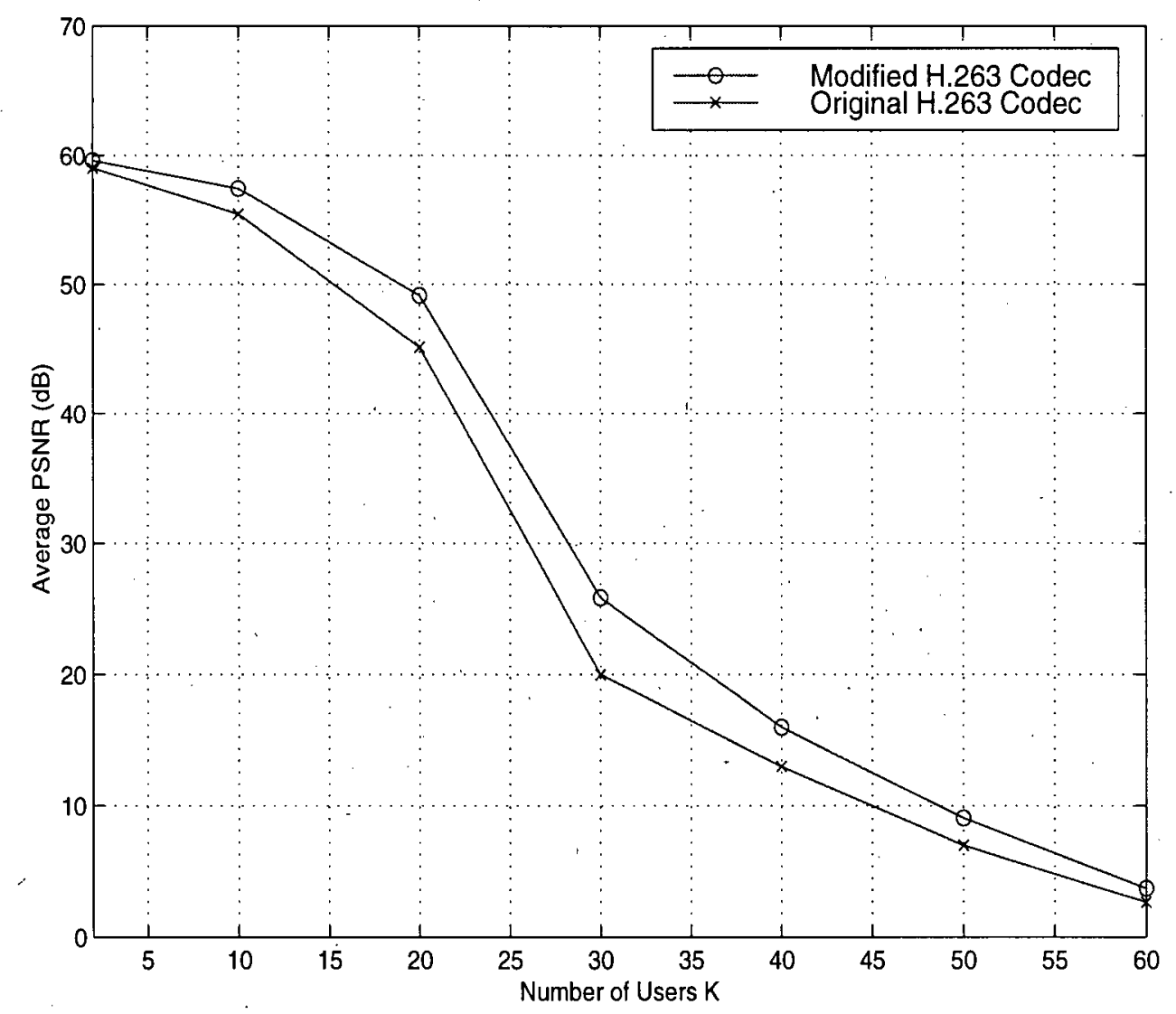

Fig. 4.10 Video Performance of Modified H.263 Codec in Single-Cell CDMA System

In Fig. 4.11, the video transmission performance is evaluated against different number of resolvable paths by the RAKE receiver.As it can be observed, the PSNR performance improves as 


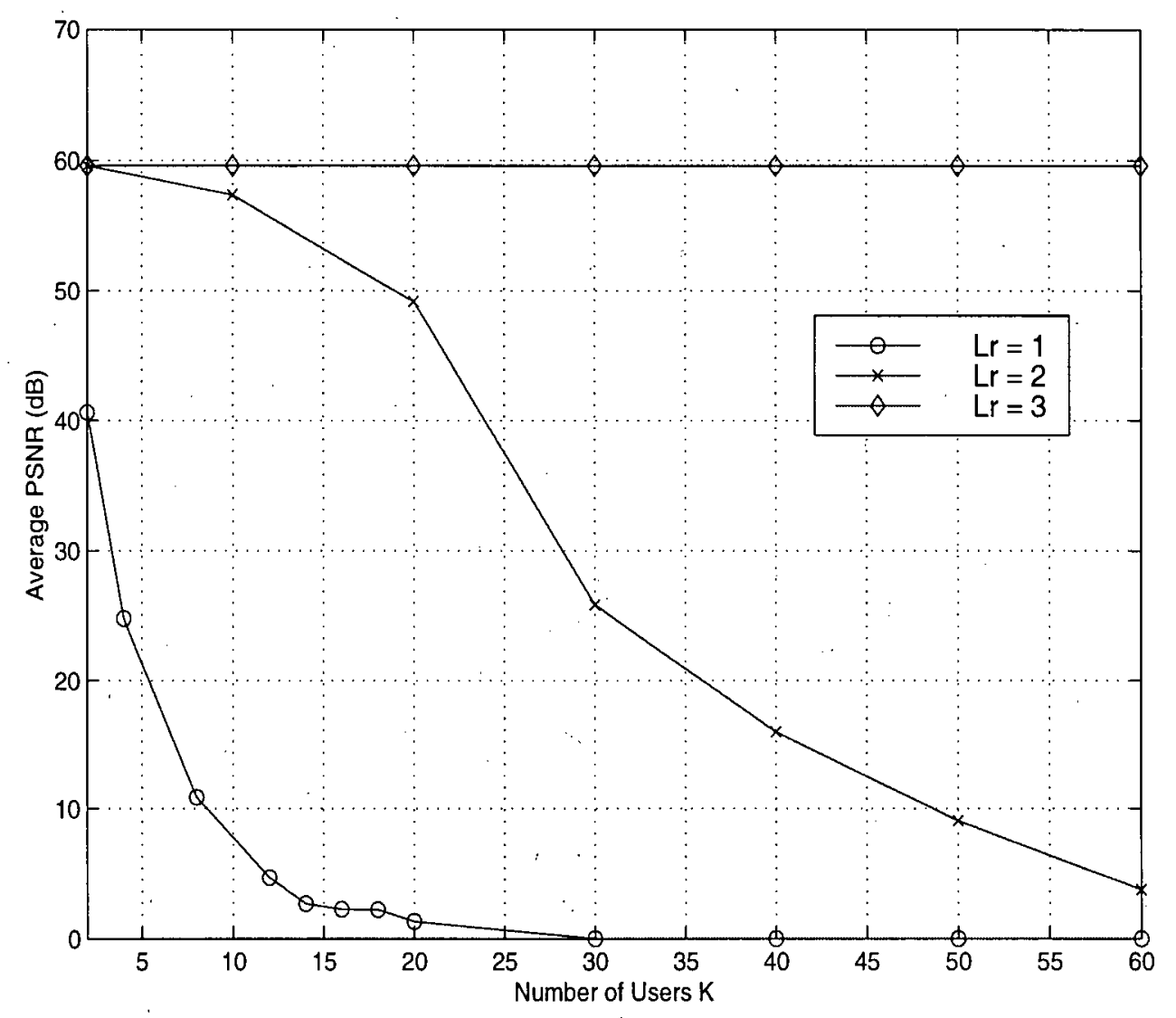

Fig. 4.11 PSNR Performance with Various Number of RAKE Fingers

$L_{r}$ increases from 1 to 3 . This coincides with the theoretical optimal value of $L_{r}=L$ for coherent demodulation because a coherent $L_{r}$-finger RAKE with perfect estimates of the channel tap weights is equivalent to a maximal ratio combiner with $L_{r}$ th-order diversity [26]. In fact, the improvements are quite substantial with each increase in the number of resolving paths. For the case of $L_{r}=1$ (single correlator receiver), the recovered video data is so corrupted that both FEC offered by the modified H.263 video codec and half-rate convolutional coder of IS-95 are unable to provide enough error recovery, resulting in relatively poor PSNR performance. For $L_{r}=2$, the quality of video is acceptable at PSNR $=40 \mathrm{~dB}$ for up to about 25 users. In the case of 
$L_{r}=L=3$, there is virtually no degradation of the video sequence quality for up to 60 users due to the fact that the channel coding is able to correct almost all of the errors. It is worth noticed that three-path diversity is indeed used in practical CDMA forward link systems [55].

Fig. 4.12 shows the PSNR performance of the video transmission under different channel conditions with Nakagami fading figure $m=1,2$ and 3. The fading. figure $m$ is related to the

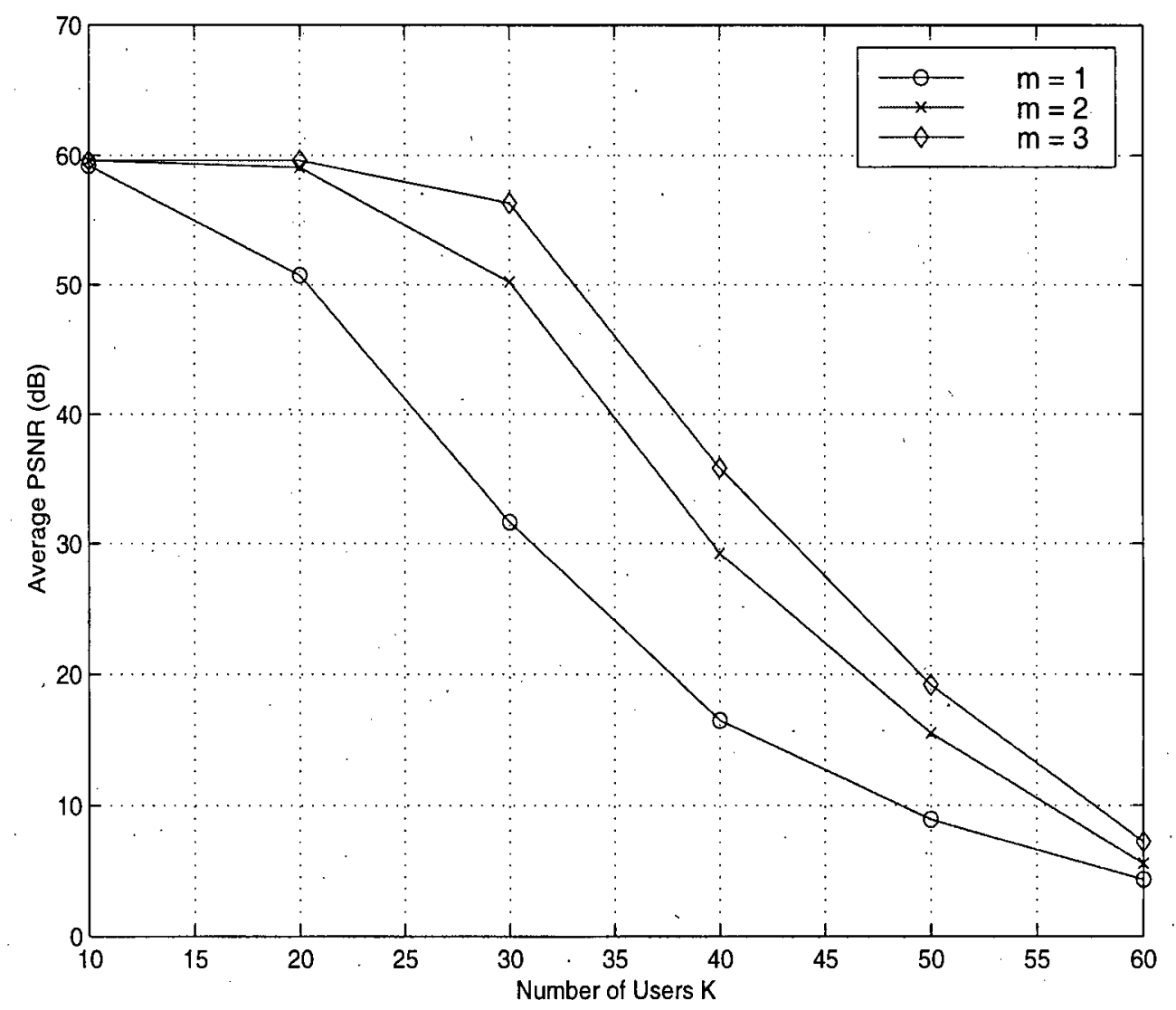

Fig. 4.12 PSNR Performance with Different Fading Figures

severity of fading. Increasing value of $m$ corresponds to less amount of fading. As the value of $m$ increases, we observe that there is significant improvement in the video quality in terms of PSNR because of the diminishing severity of the fading. The gaps in PSNR among different $m$-value 
cases become smaller when the number of users $K$ gets larger. This is because the increasing multiple-access interference has become the dominant factor in the degradation of the video transmission over the effects of fading of the channels.

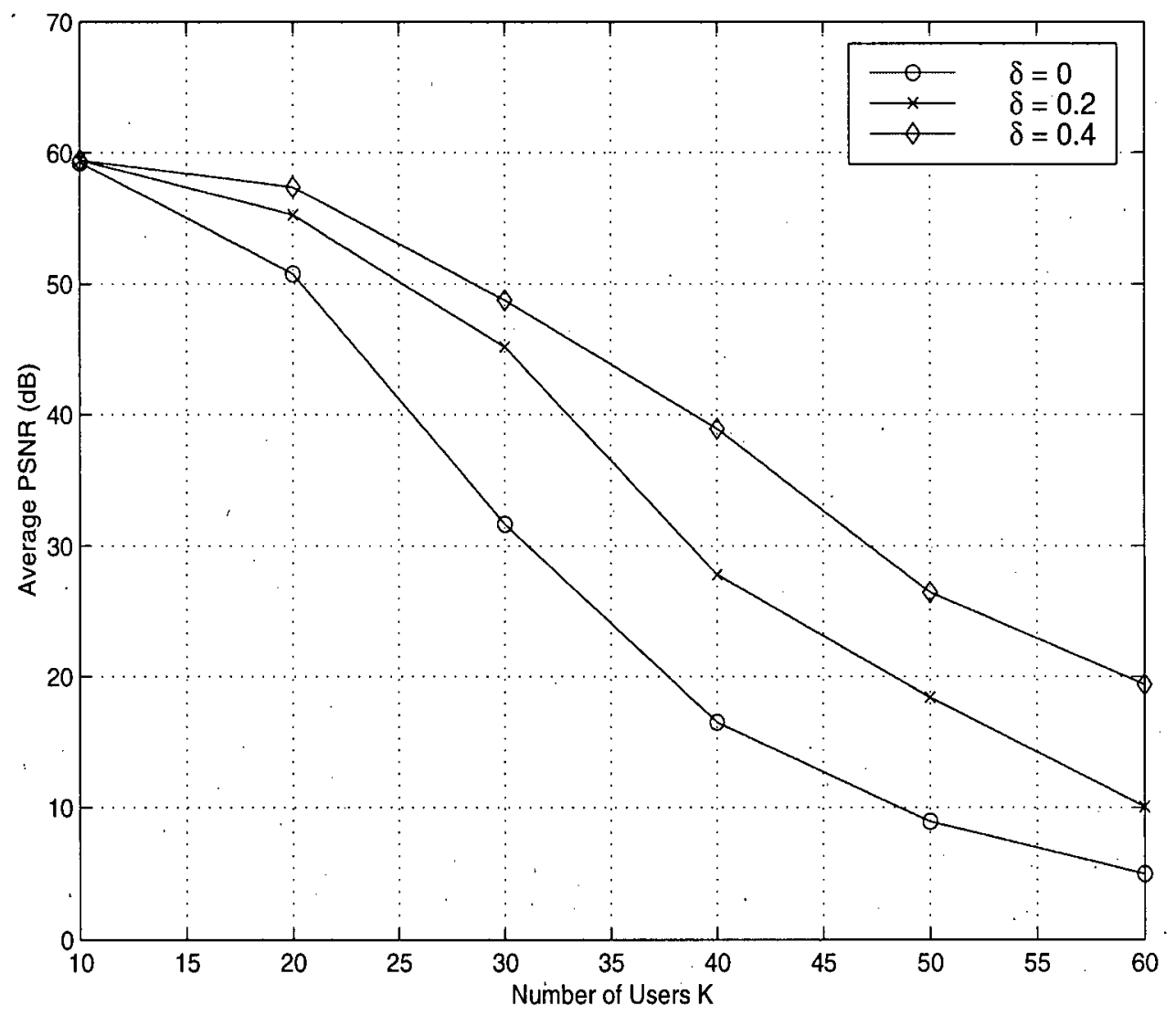

Fig. 4.13 PSNR Performance with Different Logarithmic MIP Exponents

Fig. 4.13 illustrates the effects of different multipath intensity profiles (MIP) of the signal on the PSNR performance where we consider cases of logarithmic exponent values $\delta=0,0.2,0.4$. Without loss of generality, we assume that the multipath signals have normalized power in their first path, i.e. $\Omega_{0}=1$. For $\delta=0$, it is equivalent to a constant MIP, i.e. all the multipaths have the same average power. $\delta$ of 0.2 and 0.4 correspond to multipath signal power 
set of $\{0 \mathrm{~dB},-0.86 \mathrm{~dB},-1.74 \mathrm{~dB}\}$ and $\{0 \mathrm{~dB},-1.74 \mathrm{~dB},-3.47 \mathrm{~dB}\}$, in the order of arrivals, respectively. The decreasing power in the multipath signals has two major effects when multipath signals are partially resolved $\left(L_{r}<L\right)$ as in these cases. Firstly, there is less interference arriving at the receiver. This is especially true for the self-noise due to the power reduction of delayed versions of the reference user's signal. At the same time, the sum of the desired signal power decreases as well. As shown in the figure, the average PSNR performance shows improvement for increased values of $\delta$. This happens because the reduced multiple-access and self-noise interference have a higher impact on the system performance than the reduced desired signal power does.

In Fig. 4.14, we present results showing the impact of Doppler spread on the system performance. We consider cases of mobile velocity at $100 \mathrm{~km} / \mathrm{hr}, 50 \mathrm{~km} / \mathrm{hr}$ and $5 \mathrm{~km} / \mathrm{hr}$ to represent different scenario of handset usage. The first two cases correspond to $B_{d} T=2.8 \times 10^{-3}$ and $1.4 \times 10^{-3}$ which characterize fast fading, whereas the last case corresponds to $B_{d} T=1.6 \times 10^{-5}$ which characterizes slow fading. Average duration of fades is inversely proportional to $B_{d} T$ product. Thus, fast fading cases have shorter average fade duration than slow fading. As observed from the curves, higher mobile velocity gives better average PSNR performance. The reason is that the error control used in the system, either $\mathrm{BCH}$ or convolutional coding, is designed to combat random errors. Thus, for a fixed degree of interleaving, higher velocity corresponds to shorter fade durations and thus shorter error bursts, which in turns facilitate error correction by the channel coding. The opposite applies to situations of slower speed, of which longer error bursts make channel coding ineffective, thus the resulting degrade in PSNR performance.

Fig. 4.15 shows the average PSNR performance of the video transmission under channels with non-identical $m$ values. Due to the random nature of the propagation channel, it is more 


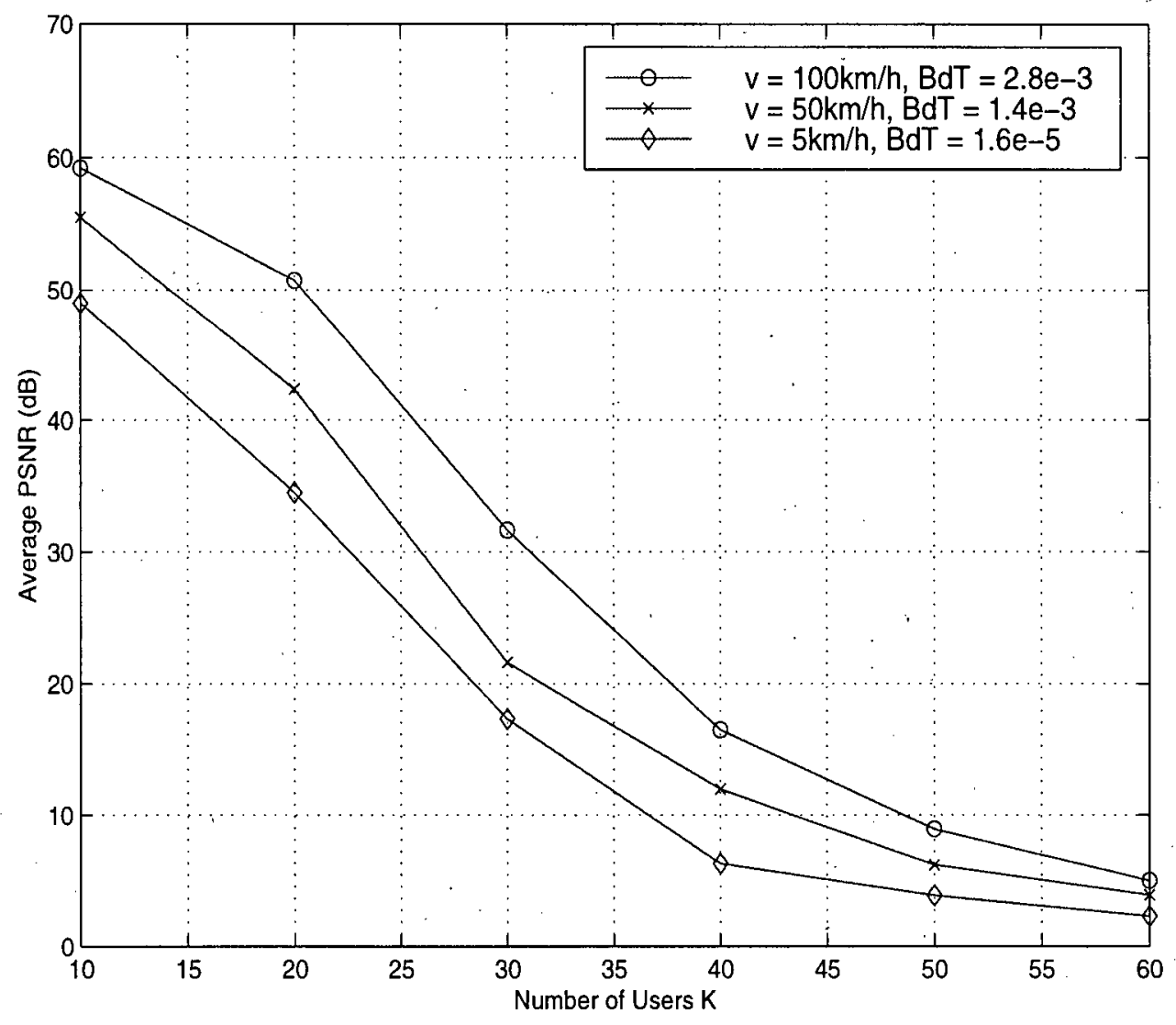

Fig. 4.14 PSNR Performance with Different Mobile Velocities

realistic to assume that the fading figures $m$ may be different for different multipaths. This may well be the case in an actual mobile link, since the radio waves take different paths and may undergo different fading before arriving at the receiver. Moreover, it is also reasonable to assume that the signal from the initial path experiences less severe fading. It is because the signal may either come from a direct wave or reflected wave with relatively less scattering than those from subsequent multipaths. As typical examples, we have considered three multipath fading scenario in terms of $m$ values in the order of signal arrivals namely, $\{1.0,1.0,1.0\},\{2.0,1.5,1.0\}$ and $\{3.0,2.0,1.0\}$. For the case of multipath $m$ values equal to $\{1.0,1.0,1.0\}$, it represents a 


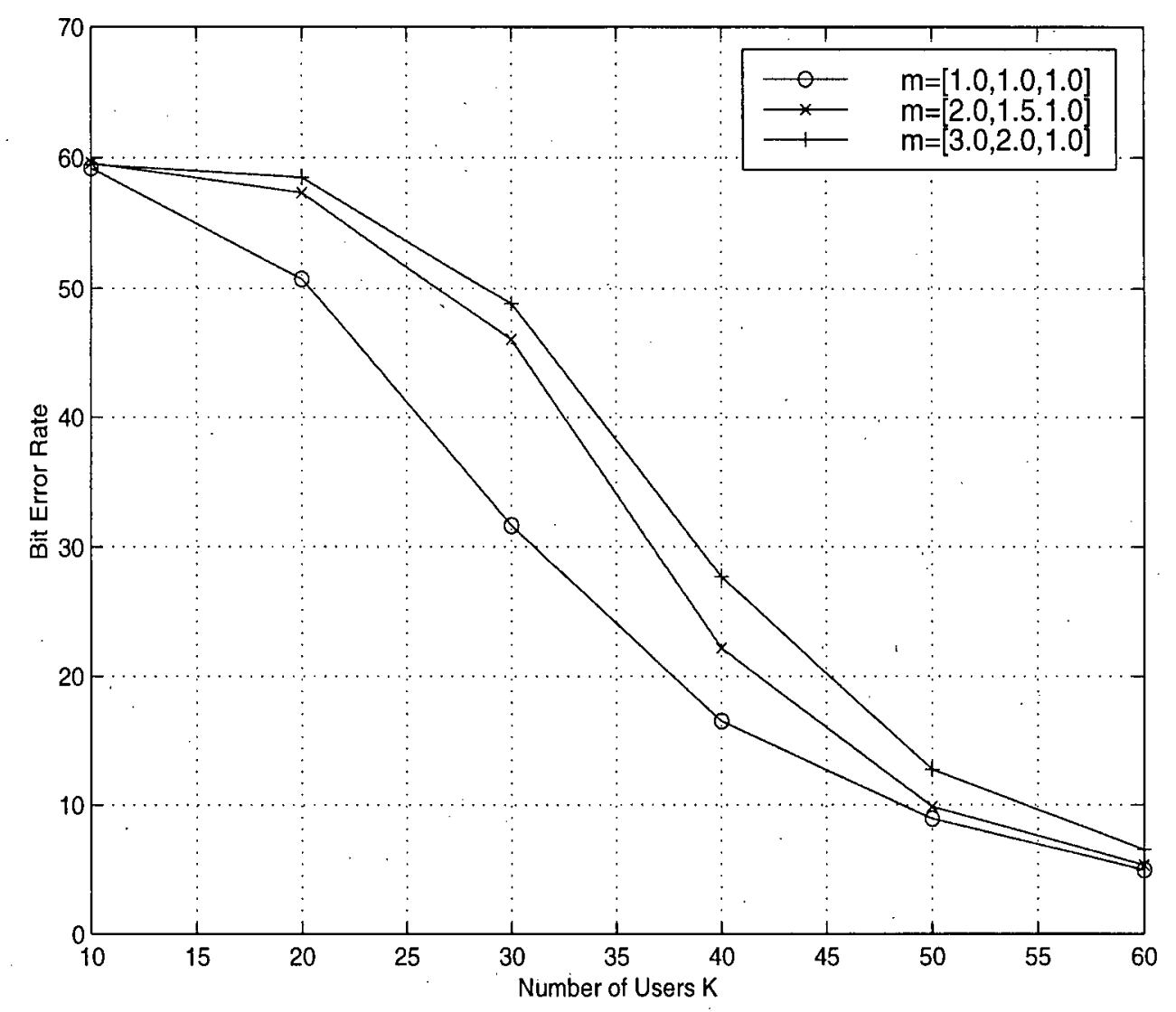

Fig. 4.15 PSNR Performance with Non-Identical Multipath Fading for Each Path

channel in which all multipaths exhibit Rayleigh fading. As observed from the graph, it results in the lowest PSNR performance. Improved PSNR performance of about $7 \mathrm{~dB}$ for 25 system users is obtained in the case of multipath $m$ values equal to $\{2.0,1.5,1.0\}$. It is due to the fact that the first and second paths have relatively less severe fading. Finally, for the case of multipath $m$ values equal to $\{3.0,2.0,1.0\}$, it gives the best video performance among all cases in the simulation. Again, it is because of the still lower average fading intensity of the multipath signals.

Finally, we have investigated the impact of imperfect estimation of the channel tap weights on the video transmission quality. As previously mentioned, in the IS-95 CDMA forward 
link, channel estimation is achieved with the aid of a pilot tone sent from the base station to the receiver. In practice, when fading is sufficiently slow, relatively good channel estimates can be obtained. However, in situations where fading is relatively fast, there can be inaccuracies in the estimation process. In order to evaluate the effect of deviations in the channel tap weights estimation on video transmission performance, we assumed an estimation error with Gaussian distribution. In the computer simulations, the exact channel fading amplitudes are deviated with Gaussian variance of different values to generate the imperfect channel tap weight estimations.

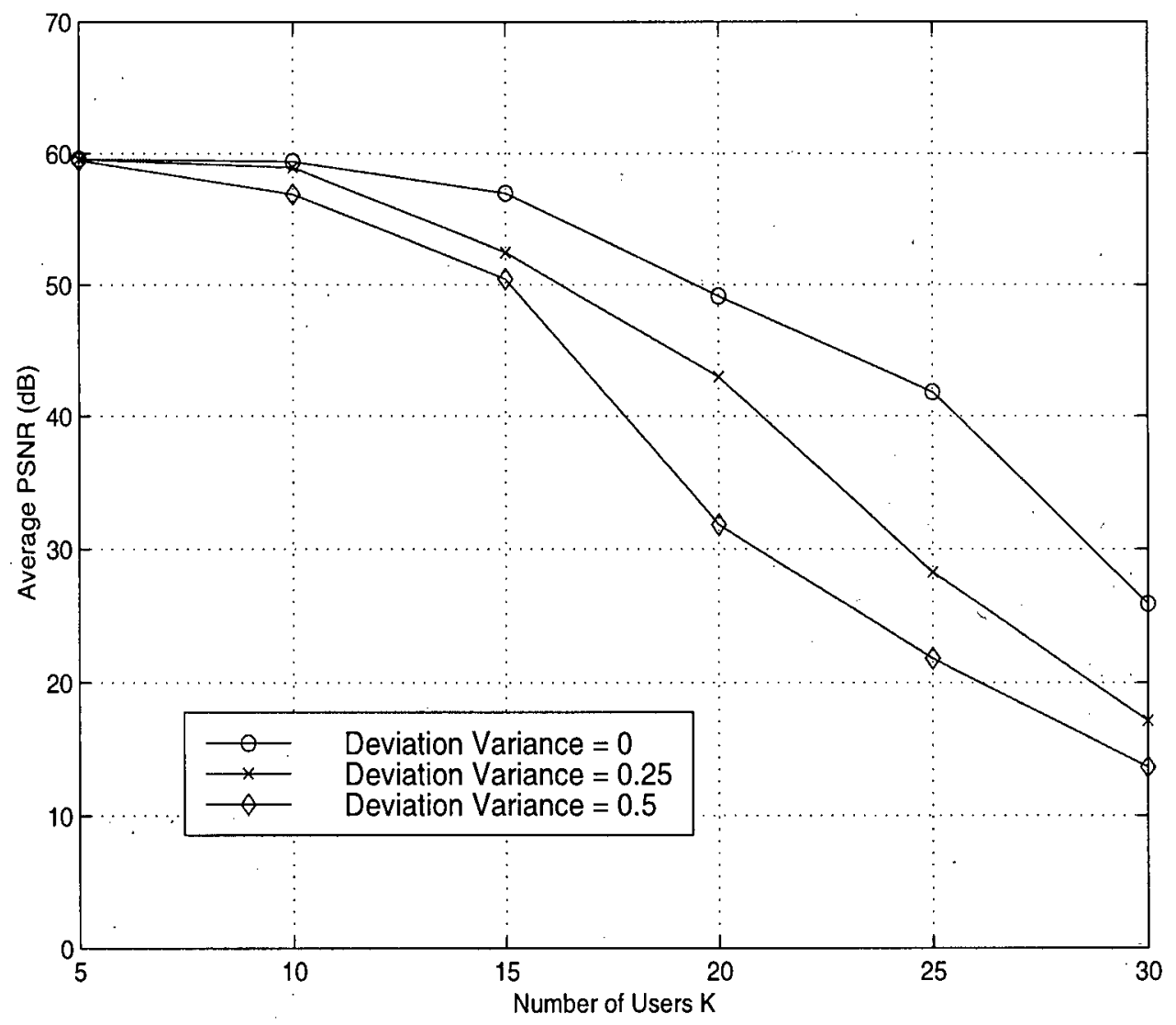

Fig. 4.16 PSNR Performance with Imperfect Channel Estimation As illustrated in In Fig. 4.16, we compare two cases of imperfect estimation with error of 
variance 0.25 and 0.5 , and include the case of perfect channel estimation for reference purposes. As seen from the results, imperfect channel tap weights estimation could degrade PSNR performance considerably. For example, let us consider the case of having 50 users in the system, receiver with perfect channel estimation can provide acceptable video quality of PSNR at about $50 \mathrm{~dB}$. However, the video PSNR values drops to about $7 \mathrm{~dB}$ and $18 \mathrm{~dB}$ for receivers with channel estimation error of variance of 0.25 and 0.5 , respectively. Therefore, we observe that the effect of channel estimation error could have a significant effect on the video transmission performance of CDMA systems.

\subsection{Conclusions}

In this section, we have investigated the video transmission performance of modified H.263 encoded data in a single-cell CDMA system over correlated Nakagami fading channels for the forward link. The DS-SS CDMA system, fading channel model and RAKE receiver structure were described. Mathematical analysis for the CDMA forward link was presented, and its results were compared with those obtained from computer simulation. It was found that Gaussian approximation provides fairly optimistic BER prediction for the single-cell forward link system. Through simulations, we illustrated that concatenated coding scheme provides superior BER performance over non-concatenated scheme for the forward link, thus justifying its applications in the forward link of the IS-95 CDMA systems. In the last section, we presented the PSNR performance of the integrated CDMA forward link video transceiver system through software simulation method. We have shown that the optimal value of $L_{r}=L$ for coherent RAKE receiver. PSNR performance improves with larger values of fading figures, MIP exponents and $B_{d} T$ products. We also illustrated that the effects of unequal fading for individual multipaths and 
imperfect channel estimations can be significant to PSNR performance. 


\section{Chapter 5 FORWARD LINK VIDEO TRANSMISSION IN MULTIPLE-CELL CDMA SYSTEMS}

\subsection{Introduction}

It is well known that, the cellular concept is being used in wireless multiple access systems to increase capacity by reusing radio resources in different cells. While TDMA and FDMA techniques must provide different frequency allocation for contiguous cells, CDMA systems can reuse the same entire spectrum for all cells, thus greatly simplify frequency planning [5]. Unlike the capacities of TDMA and FDMA systems, which are primarily bandwidth limited, CDMA capacity is primarily limited by interference level [53]. In fact, the capacity is inversely proportional to the amount of interference. For a multiple-cell environment, in addition to the usual AWGN, self-noise interference, and multiple-access interference from users present in the reference cell, there is additional interference contributed from users from neighboring cells. In this chapter, we will investigate the effects of this multiple-cell interference on the video transmission performance of a CDMA forward link.

After this introduction, in Section 5.2, we first present the multiple-cell configuration model, which consists of the first two tiers of surrounding cells. In Section 5.3, we introduce the channel model for multiple-cell systems that takes large-scale attenuation into account. In Section 5.4, the DS-SS CDMA system model for multiple-cell environment is described. In Sections 5.5 and 5.6, we present the mathematical analysis of the BER performance for the multiple-cell CDMA forward link. In Section 5.7, we describe the computer simulation model used in Monte Carlo method. In Sections 5.8 and 5.9, numerical results for the BER performance of the CDMA forward link and the PSNR performance of the transmitted video over the proposed multiple-cell 
CDMA system are presented and discussed. Finally, we complete the chapter with conclusions in Section 5.10.

\subsection{Multiple-Cell Configuration Model}

In this section, we describe the configuration model for multiple-cell systems used in this thesis.

Fig. 5:1 shows the CDMA cellular model under consideration which takes into account the

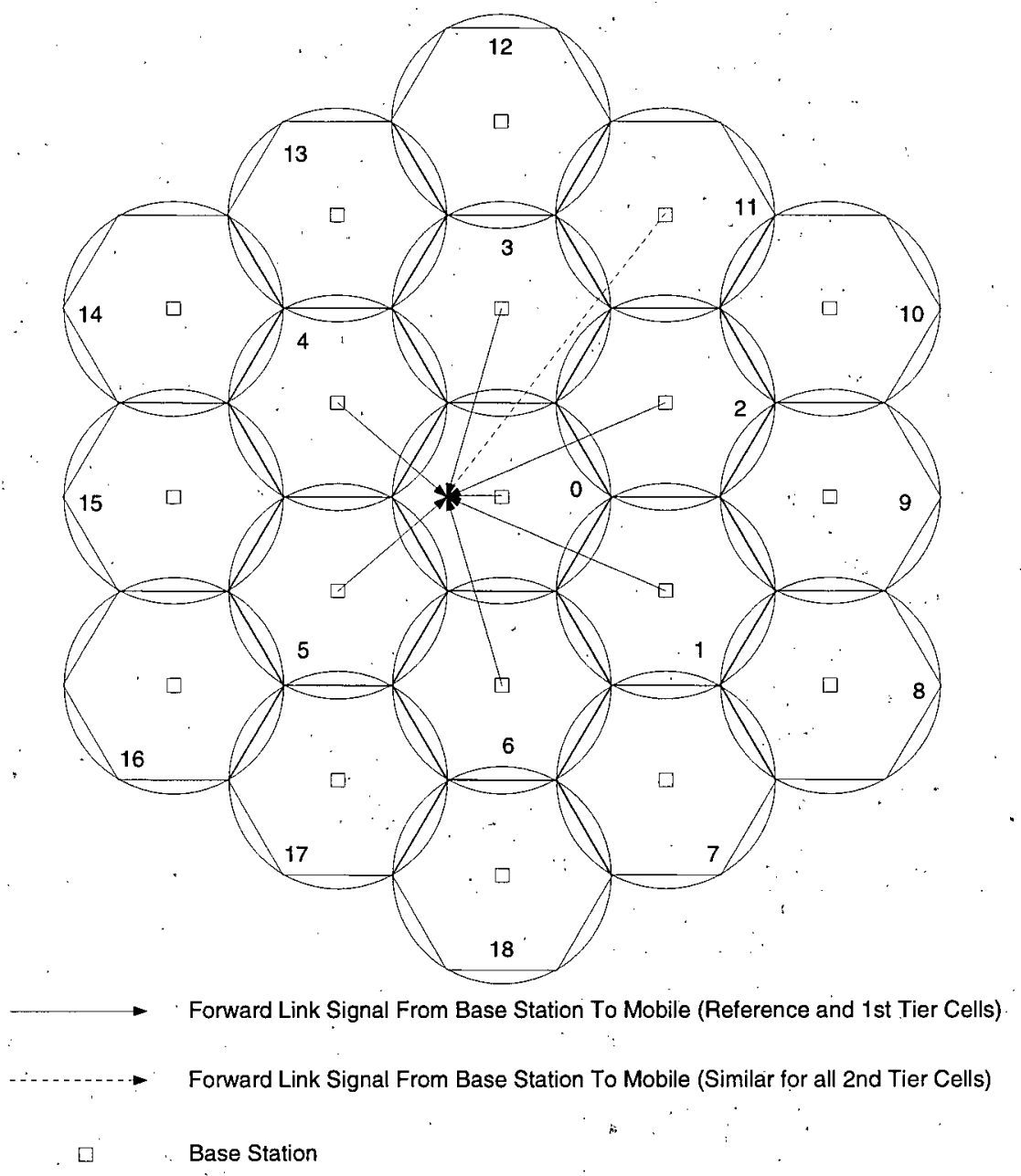

Fig. 5.1 Multiple-Cell Configuration Model

multiple-cell interference coming from the 18 surrounding cells of the first two tiers. Cells are 
numbered from 0 to 18 , with number 0 denoting the reference cell, and 1 to 18 denoting the surrounding cells. The signals from cells farther away are neglected in the investigation. It is justified by the fact that they suffer greater attenuation due to path loss. To simplify analysis, each cell is assumed to be circular with uniform size, and that each base station (BS) is located at the centre of the cell. Also, the reference mobile user is assumed to be equally likely located anywhere within the reference cell. In Fig. 5.1, the reference mobile, located at the convergence point of arrows representing the forward link signals, receives radio signals from the reference base station (denoted by the square at cell 0 ) as well as from the surrounding 18 base stations (denoted by the squares at cell 1 to 18 ).

\subsection{Channel Modeling}

In this section, we describe the radio propagation model for signals travelling in a multiple-cell environment. To model the mobile channel for such a cellular environment, the effects of largescale fading due to shadowing and path loss have to be taken into account. We employ the largescale fading model as discussed in Chapter 2, which assumes that the average signal attenuation is the product of the $\gamma$ th power of distance and a log-normal random variable (see (2.1)). Thus; we can relate the transmitted average signal power $P_{q}$ and the received average signal power $\hat{P}_{q}$ from the $q$ th BS to the reference mobile as

$$
\hat{P}_{q} \propto P_{q} d_{q}^{-\gamma} 10 \zeta_{q} / 10
$$

where $d_{q}$ is the distance between the reference mobile and the $q$ th base station, $\gamma$ is the path loss exponent, and $\zeta_{q}$ is a Gaussian random variable with zero mean and standard deviation $\sigma_{q}$. We will use $\gamma=4$ for the power law and $\sigma_{q}=8 \mathrm{~dB}$ for the standard deviation of the log-normal shadowing random variable $\zeta_{q}$, as it has been suggested in past literature [41], [53]. 
For small-scale fading, we employ the same frequency-selective fading model as described in Section 4.3 for signals transmitted from each base station. Each forward link signal from the $q$ th cell is modeled by a tapped delay line (Fig. 4.3) with statistically independent timevariant tap weights $\left\{c_{q l}(t)\right\}$, where $q=0,1, \ldots, Q-1$ and $l=0,1, \ldots, L-1$. The variable $Q$ is the total number of cells (assumed to be 19) and $L$ is the total number of multipaths upper bounded by (4.1). The magnitudes of the tap weights $\left|c_{q l}(t)\right| \equiv \beta_{q l}(t)$ for the $l$ th signal paths from the $q$ th cells are assumed to be Nakagami- $m$ distributed and the corresponding phases $\angle c_{q l}(t)=\theta_{q l}(t)$ to be uniformly distributed over $[0,2 \pi)$

\subsection{Multiple-Cell DS-SS CDMA System Model}

In this section, we describe the direct sequence spectrum spreading scheme for the CDMA systems in a multiple-cell environment, which is similar to that of the single-cell systems as described in Section 4.2. The spreading codes are obtained from the concatenation of Walsh codes with a PN sequence. For a multiple-cell CDMA system, the same PN sequence is shared by all BS. Each BS, however, uses a different time-shifted versions of this common PN sequence for Walsh code concatenation [56]. Mobile receivers detect the amount of time offsets of the PN code to identify one BS from another.

We assume the signals transmitted from different BS are chip-synchronized, i.e. signal delay $\tau=k T_{c}$ for some integer $k$. In the actual IS-95 CDMA network, the system time is synchronous to that of Universal Time Coordination (UTC), which uses the same time origin as Global Positioning System (GPS) [55]. All base stations in the CDMA system synchronize to the same system time accurately, as it is crucial for proper system operations. Thus, it is justified to assume that the base stations transmit signals in a chip-synchronized manner. 


\subsection{Multiple-Cell CDMA Forward Link BER Performance Analysis}

For a CDMA forward link operating in a multiple-cell environment, the transmitted signal for the $k$ th user from the $q$ th cell is a phase-coded carrier expressed as

$$
s_{q}^{(k)}(t)=\sqrt{2 P_{q}} a_{q}^{(k)}(t) b_{q}^{(k)}(t) \cos \left(\omega_{c} t+\phi_{q}\right)
$$

where $a_{q}^{(k)}(t)$ is the spreading code sequence of the $k$ th user from the $q$ th cell;

$$
a_{q}^{(k)}(t)=\sum_{j=-\infty}^{\infty} a_{q j}^{(k)} p_{a}\left(t-j T_{c}\right), \quad a_{q j}^{(k)} \in\{-1,1\}
$$

and $b_{q}^{(k)}(t)$ is the data waveform,

$$
b_{q}^{(k)}(t)=\sum_{j=-\infty}^{\infty} b_{q j}^{(k)} p_{b}(t-j T), \quad b_{q j}^{(k)} \in\{-1,1\}
$$

In (5.2), $P_{q}$ is the transmitted power from the $q$ th base station, $\omega_{c}$ is the common radian carrier frequency, and $\phi_{q}$ is the initial phase of the $q$ th base station modulator uniformly distributed over $[0,2 \pi)$. In (5.3) and (5.4), $T_{c}$ is the chip duration, $T$ is the data bit duration, $p_{a}(t)$ and $p_{b}(t)$ are rectangular pulses of unit height and durations of $T_{c}$ and $T$, respectively.

The total signal transmitted by the $q$ th base station, assuming there are $K_{q}$ users belonging to the qth base station, is

$$
s_{q}(t)=\sum_{k=0}^{K_{q}-1} \sqrt{2 P_{\dot{q}}} a_{q}^{(k)}(t) b_{q}^{(k)}(t) \cos \left(\omega_{c} t+\phi_{q}\right)
$$

For a multiple-cell model, the received signal after the channel is

$$
\hat{r}(t)=\sum_{q=0}^{Q-1} \sum_{k=0}^{K_{q}-1} \sum_{l=0}^{L_{q}-1} \sqrt{2 \hat{P}_{q}} \cdot \beta_{q l} \cdot a_{q}^{(k)}\left(t-\tau_{q l}\right) \cdot b_{q}^{(k)}\left(t-\tau_{q l}\right) \cos \left(\omega_{c} t+\varphi_{q l}\right)+n(t)
$$

In (5.6), $Q$ is the total number of cells, $K_{q}$ is the total number of users in the $q$ th cell, and $L_{q}$ is 
the number of multipaths for the signal from the $q$ th base station, which is a random value upper bounded by (4.1). Similarly to the single cell system model, $\hat{P}_{q}$ is the received signal power from the $q$ th cell as defined in (5.1). $\varphi_{q l}=\phi_{q}+\theta_{q l}+\omega_{c} t \cdot \tau_{q l}$ is the phase of the $l$ th path from the $q$ th base station's signal, where $\phi_{q}$ is the original phase, $\theta_{q l}$ is the channel phase shift and $\tau_{q l}$ is the multipath time delay for the $l$ th path from the corresponding cell, respectively. Each path is assumed to fade independently with fading coefficient $\beta_{q l} e^{j \theta_{q l}}$ in which the amplitude $\beta_{q l}$ follows a Nakagami- $m$ distribution and the phase $\theta_{q l}$ follows a uniform distribution over $[0,2 \pi)$. The final term $n(t)$ is the AWGN process with two-sided power spectral density of $\eta_{o} / 2$.

Equivalent to the single-cell system analysis in Section 4.6, the response of the coherent RAKE receiver at each sampling time can then be expressed as

$$
\begin{aligned}
\hat{U} & =\sum_{n=0}^{L_{r}-1} \int_{n T_{c}}^{T+n T_{c}} \hat{r}(t) \beta_{q n} a_{0}^{(0)}\left(t-n T_{c}\right) \cos \left(\omega_{c} t+\varphi_{n}\right) d t \\
& =\sum_{n=0}^{L_{r}-1}\left\{\hat{S}^{(n)}+\hat{I}_{m a i}^{(n)}+\hat{I}_{s i}^{(n)}+\hat{I}_{n i}^{(n)}\right\}
\end{aligned}
$$

where $L_{r}$ is the number of resolving RAKE fingers. In (5.7), $\hat{S}^{(n)}$ is the desired signal component, $\hat{I}_{m a i}^{(n)}$ is the multiple-access interference component, $\hat{I}_{s i}^{(n)}$ is the self-noise component, and $\hat{I}_{n i}^{(n)}$ is the AWGN component at the output of each RAKE finger, respectively.

\subsection{Multiple-Cell BER Performance Analysis with Gaussian Approximation}

By following the same procedure of applying the Gaussian approximation to BER performance analysis as in Chapter 4 , we can write the response $\hat{U}$ of the reference receiver to the received signal at any sampling instant as a conditional complex Gaussian random variable with conditional mean of the desired signal component $\hat{U}_{s}$, and conditional variance equals to the sum 
of all interference terms, i.e.,

$$
\hat{\sigma}_{S}^{2}=\sum_{n=0}^{L_{r}-1}\left(\hat{\sigma}_{M A I, \mathrm{n}}^{2}+\hat{\sigma}_{S I, n}^{2}+\hat{\sigma}_{N I, n}^{2}\right)
$$

In order to find the desired signal component $\hat{U}_{s}$, we modify $U_{s}$ in (4.21) so that the large-scale fading effect is taken into account. The original signal power $P$ of the reference user is replaced by the attenuated power $\hat{P}_{0}$ of the reference user located at cell 0 as given by (5.1) such that

$$
\hat{U}_{s}=\sqrt{\frac{\hat{P}_{0} T^{2}}{2}} \cdot \sum_{n=0}^{L_{r}-1}\left\{\beta_{0 n}\right\}^{2}
$$

where $\beta_{0 n}$ is the fading amplitude of the $n$th resolvable path of the reference base station.

To find the total interference $\hat{\sigma}_{S}^{2}$ for the multiple-cell systems, we apply certain modifications on the individual components of total interference $\sigma_{S}^{2}$ in the single-cell scenario. For multiple-access interference (MAI), in conjunction with the amount caused by intra-cell users, multiple-cell systems have additional interference generated by $K_{q}$ number of users from each of the surrounding cells, for $q=1, \ldots, Q-1$. Moreover, since signals from different cells reach the reference mobile through different channel environment, they each have different attenuation and multipath intensity profiles. To account for these differences, the $\sigma_{M A I, n}^{2}$ term in (4.18) is modified by adding a second term to represent the summation of multiple-access interference from cell 1 to cell $Q-1$. Also, we replace the common power $P$ of the reference cell by the attenuated signal power $\hat{P}_{q}$ for the $q$ th cell as given by (5.1). Finally, the multipath intensity profile $\Omega_{l}$ for the reference cell in (4.18) is replaced by a more generalized term $\Omega_{q l}$ denoting for the multipath intensity profile for the qth cell, which is defined as 


$$
\Omega_{q l}=\Omega_{q 0} e^{-l \delta_{q}}, \quad \delta_{q} \geq 0
$$

where $\Omega_{q 0}$ is the first path average signal strength and $\delta_{q}$ is the rate of average power decay for the $q$ th base station signal propagation, respectively.

After accommodating all these changes, we obtain the conditional MAI variance at the $n$th RAKE finger for a multiple-cell system as

$$
\hat{\sigma}_{M A I, n}^{2}=\frac{\hat{P}_{0} T^{2}}{4 N}\left\{\beta_{0 n}\right\}^{2} \cdot \sum_{k=1}^{K_{0}-1 L_{0}-1} \sum_{l=0} \Omega_{0 l}+\sum_{q=1}^{Q-1} \frac{\hat{P}_{q} T^{2}}{4 N}\left\{\beta_{0 n}\right\}^{2} \cdot \sum_{k=0}^{K_{q}-1} \sum_{l=0}^{L_{q}-1} \Omega_{q l}
$$

where the first term represents MAI from within the reference cell, and the second term represents MAI from the surrounding cells. The summation sign of users changes from $k=1, \ldots, K_{0}-1$ for the reference cell user MAI term to $k=0, \ldots, K_{q}-1$ for the surrounding cell MAI term, in order to account for an additional MAI interferencing users from each of the surrounding cells.

Self-interference is obtained by modifying (4.19) in a similar manner. The common signal power $P$ in (4.19) is substituted by the attenuated signal power $\hat{P}_{0}$ of the reference cell. The MIP term $\Omega_{l}$ is replaced by the MIP term for the reference cell denoted as $\Omega_{0 l}$, which is defined in (5.10), to give

$$
\hat{\sigma}_{S I, n}^{2}=\frac{\hat{P}_{0} T^{2}}{4 N} \cdot\left\{\beta_{0 n}\right\}^{2} \sum_{l=1}^{L_{0}-1} \Omega_{0 l}
$$

The AWGN component $\hat{\sigma}_{N I, n}^{2}$ for the multiple-cell environment is obtained by substituting the fading amplitude $\beta_{n}$ by $\beta_{0 n}$ to denote for the reference cell, which gives

$$
\hat{\sigma}_{N I, n}^{2}=\frac{T \eta_{o}}{4} \cdot\left\{\beta_{0 n}\right\}^{2} .
$$

The received SNR with a RAKE receiver resolving $L_{r}$ paths for a multiple-cell system is defined 
as

$$
\operatorname{SNR}=\frac{\left(\hat{U}_{s}\right)^{2}}{\sum_{n=0}\left[\hat{\sigma}_{M A I, n}^{2}+\hat{\sigma}_{S I, n}^{2}+\hat{\sigma}_{N I, n}^{2}\right]}
$$

After substituting $\hat{U}_{s}, \hat{\sigma}_{M A I, n}^{2}, \hat{\sigma}_{S I, n}^{2}$, and $\hat{\sigma}_{N I, n}^{2}$ into (5.14), and performing some straightforward simplifications, we obtain

$$
\begin{aligned}
\mathrm{SNR}= & \frac{2 \hat{P}_{0} \cdot\left(\sum_{n=0}^{L_{r}-1}\left\{\beta_{0 n}\right\}^{2}\right)^{2}}{\sum_{n=0}^{L_{r}-1}\left(\frac{\hat{P}_{0}}{N} \cdot\left\{\beta_{0 n}\right\}^{2} \sum_{k=1}^{K_{0}-1} \sum_{l=0}^{L_{0}-1} \Omega_{0 l}+\sum_{q=1}^{Q-1} \frac{\hat{P}_{q}}{N} \cdot\left\{\beta_{0 n}\right\}^{2} \sum_{k=0}^{K_{q}-1} \sum_{l=0}^{L_{q}-1} \Omega_{q l}\right.} \\
& \left.+\frac{\hat{P}_{0}}{N} \cdot\left\{\beta_{0 n}\right\}^{2} \sum_{l=1}^{L_{0}-1} \Omega_{0 l}+\frac{\eta_{o}}{T} \cdot\left\{\beta_{0 n}\right\}^{2}\right)
\end{aligned}
$$

In an interference-limited CDMA system, spreading codes of multiple users constitute the dominant source of interference when compared to AWGN. This is especially true for a multiplecell system where typically the number of users is large. In light of the relative insignificance of the AWGN term $\sigma_{N I, n}^{2}$, the last summation term of the denominator of (5.15) can be dropped to simplify the mathematical analysis.

Since we are only interested in the relative received power from the $q$ th base station to that of the reference base station, we introduce the attenuation factor of the signal power from the $q$ th $(q \neq 0)$ base station relative to that of the reference base station $(q=0)$ as

$$
\rho_{q}=\frac{d_{q}^{-\gamma} 10^{\zeta_{q} / 10}}{d_{0}^{-\gamma} 10^{\zeta_{0} / 10}}
$$

as illustrated in Fig. 5.2. In this figure, cell $q$ represents any of the 18 surrounding cells in the first 


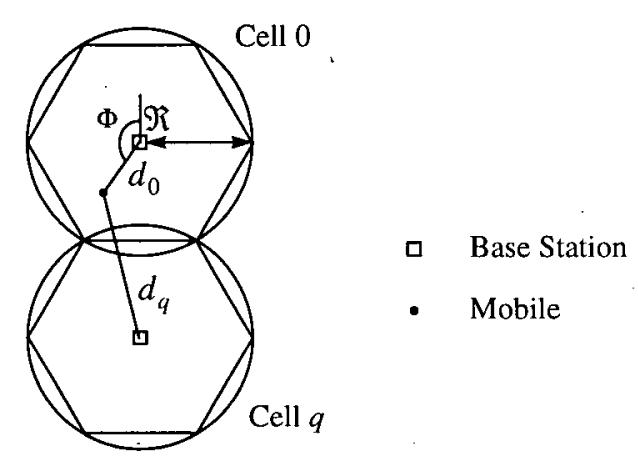

Fig. 5.2 Cell Geometry Model

two tiers, $\mathfrak{R}$ is the cell radius and $\Phi$ is the mobile location angle with respect to the base station. In addition, assuming identical MIP and uniform average number of users among different base stations, i.e.

$$
\begin{gathered}
\Omega_{q l}=\Omega_{0 l}=\Omega_{l} \\
K_{q}=K_{0}=K
\end{gathered}
$$

then (5.15) can be written as

$$
\mathrm{SNR}=\frac{\sum_{n=0}^{L_{r}-1}\left\{\beta_{0 n}\right\}^{2}}{\frac{1}{2 N}\left(\sum_{k=1}^{K-1} \sum_{l=0}^{L-1} \Omega_{l}+\sum_{q=1}^{Q-1} \rho_{q} \sum_{k=0}^{K-1 L-1} \sum_{l=0}^{L} \Omega_{l}+\sum_{l=1}^{L-1} \Omega_{l}\right)} .
$$

Similar to Chapter 4, the received SNR at the output of the receiver may be re-written in a more compact form as $\Upsilon_{m} S_{m}$, where

$$
\Upsilon_{m}=2 N\left((K-1) q(L, \delta)+\sum_{q=1}^{Q-1} \rho_{q} K \cdot q(L, \delta)+(q(L, \delta)-1)\right)^{-1}
$$

and 


$$
S_{m}=\frac{1}{\Omega_{0}} \sum_{n=0}^{L_{r}-1}\left\{\beta_{0 n}\right\}^{2}
$$

To obtain the area-averaged BER, mean values of the attenuation factor $\rho_{q}$ in (5.20) for different reference mobile locations need to be evaluated. We assume that the power levels transmitted by all base stations are the same, i.e. $P_{q}=P_{0}=P$ for all $q$. We further assume that the mobiles are always served by the base station with the strongest signal, i.e. $\rho_{q} \leq 1$. This assumption is well justified by the fact that this type of selection diversity is used in practical CDMA systems for soft handoff operations [55]. Under these conditions, we follow the method of Fong et al. [32] in evaluating the values of $E\left[\rho_{q}\right]$ for the first and second tier cells the results of which are

\begin{tabular}{|c|c|}
\hline 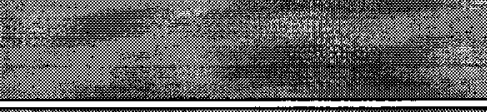 & EIP $\mid$ \\
\hline $\begin{array}{l}6 \text { cells from the ist ther } \\
9-1,06\end{array}$ & 0.07561 \\
\hline 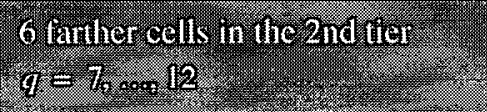 & 0.03315 \\
\hline $\begin{array}{l}\text { 6 closer cells in the } 2 \text { ndi ther } \\
q=13, \ldots, 18\end{array}$ & 0.02409 \\
\hline
\end{tabular}

Table 5.1 Area Mean of Attenuation Factor

summarized in Table 5.1. The detailed derivation of how these values have been obtained can be found in Appendix B.

After applying these values of attenuation factor on $\Upsilon_{m}$, we can then replace $\Upsilon$ and $S$ in (4.28) by $\Upsilon_{m}$ and $S_{m}$ to obtain the area-averaged BER for the multiple-cell CDMA system as

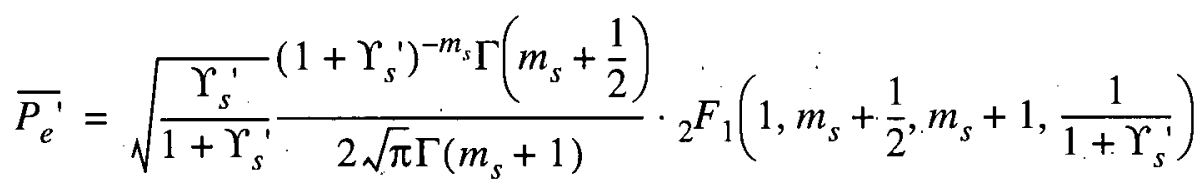


where a new term $\Upsilon_{s}^{\prime}$ introduced is defined as

$$
\Upsilon_{s}^{\prime}=\frac{\Upsilon_{m} \Omega_{s}}{2 m_{s}}=\frac{\Upsilon_{m} q\left(L_{r}, 2 \delta\right)}{2 m q\left(L_{r}, \delta\right)}
$$

Numerical results will be presented in Section 5.8 with those obtained from Monte Carlo method, of which the simulation model will be described in the next section.

\subsection{Computer Simulation Model Description}

In this section, we describe the software simulation model used for the performance evaluation employing Monte Carlo error counting techniques. Comparing to a single-cell system, additional $K_{q}$ users from $Q-1$ surrounding cells are needed to generate in the multiple-cell system simulation. The preceding bits $b_{-1}^{\left(q_{k}\right)}$ and present bits $b_{0}^{\left(q_{k}\right)}$ of the $k$ th user of the $q$ th cell (for $k=0,1, \ldots, K_{q}-1$ and $\left.q=0,1, \ldots, Q-1\right)$ are generated as random i.i.d. variables of values taken from the alphabet $\{ \pm 1\}$ with equal probabilities as in Section 4.8. The signal amplitude $\beta_{q l}$, the phase $\varphi_{q l}$, and the multipath delays $\tau_{q l}$ of the $l$ th path $\left(l=0,1, \ldots, L_{q}-1\right)$ from the $q$ th base station, as well as the AWGN random process are generated in the same manner as in the single-cell scenario. Decision output is obtained by adding the desired signal component $\hat{S}^{(n)}$, multiple-access interference $\hat{I}_{m a i}^{(n)}$, self-noise interference $\hat{I}_{s i}^{(n)}$ and AWGN interference term $\hat{I}_{n i}^{(n)}$ from the $n$th RAKE finger, for $n=0, \ldots, L_{r}-1$ where $L_{r}$ is the total number of resolvable fingers. Error count is obtained by checking the detected bit with the transmitted bit. The process is repeated to receive an adequate number of errors in order to achieve a $95 \%$ confidence interval of $\pm 5 \%$ of the average $B E R$. 


\subsection{BER Performance Evaluation Results for Multiple-Cell Systems}

Fig. 5.3 shows the BER performance of a CDMA forward link in a multiple-cell environment obtained by analytical and Monte Carlo simulation methods. As in the case of single-cell systems,

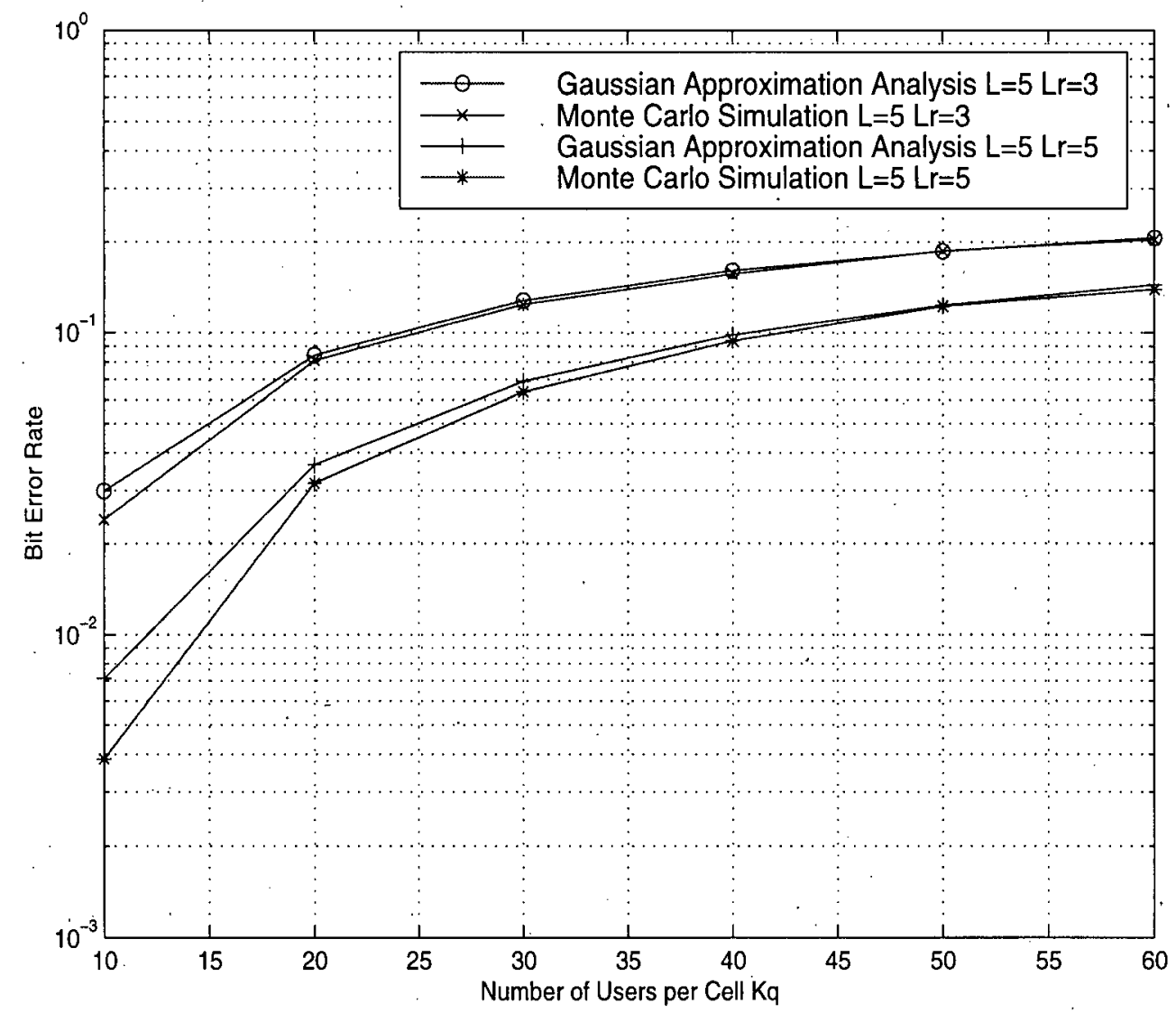

Fig. 5.3 Analysis vs. Simulation for Multi-cell System

random spreading sequences are used in both methods for comparison purposes. System parameters are chosen as $E_{b} / \eta_{0}=30 \mathrm{~dB}$ and processing gain $N=64$ as before. We assume a fading figure value of $m=2.0$ in the comparison to demonstrate the flexibility of Nakagami fading channel modeling. We consider two cases of total number of multipaths $L=5$ with different 
number of RAKE fingers $L_{r}=3$ and 5, respectively.

As shown in the figure, the BER results evaluated from the analysis using the Gaussian approximation and the Monte Carlo simulation show good agreement. This contrasts with the observation in the case of single-cell systems in Section 4.9 where we found a fair amount of BER performance discrepancies between the two approach. This improvement can be explained by the fact that in multiple-cell systems, forward link signals from different base stations exhibit independent propagation characteristics such as amplitude fluctuations, phase distortions and transmission delays, with respect to other stations. Thus, the overall interference components of a multiple-cell system consist of more independent random variables as compared to the single-cell case. As a result, the central limit theorem in the Gaussian approximation is better satisfied in the process of analyzing BER performance of the multiple-cell systems. We also observe the trend of decreasing discrepancies between the BER performance results obtained from the two methods in cases of increasing number of users per cell $K_{q}$ and higher BER values. This is similar to that has been observed in the single-cell scenario, which has been discussed and explained in Section 4.8.

\subsection{Video Transmission Performance in Multiple-Cell Systems}

In this section, we present the numerical results obtained from computer simulations of the video transmission performance over the proposed multiple-cell CDMA forward link operating in Nakagami fading channel environments. The simulated system model is similar to that of Section 4.9 , except in this case, we incorporate additional 18 surrounding cells around the reference cell in the system according to the configuration model described in Section 5.2. Each surrounding base station uses a different time-shifted version of the common PN sequence for concatenation with the Walsh codes. The signals transmitted from each base station travel through an independent 
frequency-selective channel simulated by the tapped delay line model similar to that of the singlecell case. The clocks of all base stations are assumed to be synchronized at the chip level as explained previously in Section 5.4. The results are presented in terms of average PSNR values versus number of users per cell. As in the previous chapter, each PSNR data point is generated by taking the average of 20 simulation runs using different random seeds.

Unless otherwise stated, the default values of the system parameters used to obtain the following numerical results are as follows:

- Total number of multipaths $L=3$. This value is chosen to be less than the maximum number of multipaths 5 as evaluated in Section 4.3 for the assumed maximum multipath spread of $5 \mu \mathrm{s}$. We will vary $L$ during the course of performance evaluation.

- Number of multipath resolving RAKE fingers $L_{r}=3$, as is generally used in practical CDMA systems.

- Mobile velocity is $100 \mathrm{~km} / \mathrm{hr}$ and carrier frequency is $2 \mathrm{GHz}$, resulting in a maximum Doppler frequency $B_{d}$ of about $180 \mathrm{~Hz}$. Thus, the default channel has value of $B_{d} T \approx 2.8 \times 10^{-3}$ which can be considered as exhibiting fast fading characteristics.

- Multipath intensity profile is logarithmic with default exponent $\delta=0.2$.

In Fig. 5.4, we illustrate the average PSNR performance of video transmission under mobile channels with non-identical Nakagami fading figures (the $m$ values) for each multipath. As examples, we consider three multipath fading $m$ values in the order of signal arrivals namely, $\{1.0,1.0,1.0\},\{2.0,1.5,1.0\}$ and $\{3.0,2.0,1.0\}$. The last case gives the best PSNR performance because it has the lowest average fading severity.

In Fig. 5.5, we have evaluated the video performance for multiple-cell systems where multipath signals transmitted from base stations of different tiers go through mobile fading 


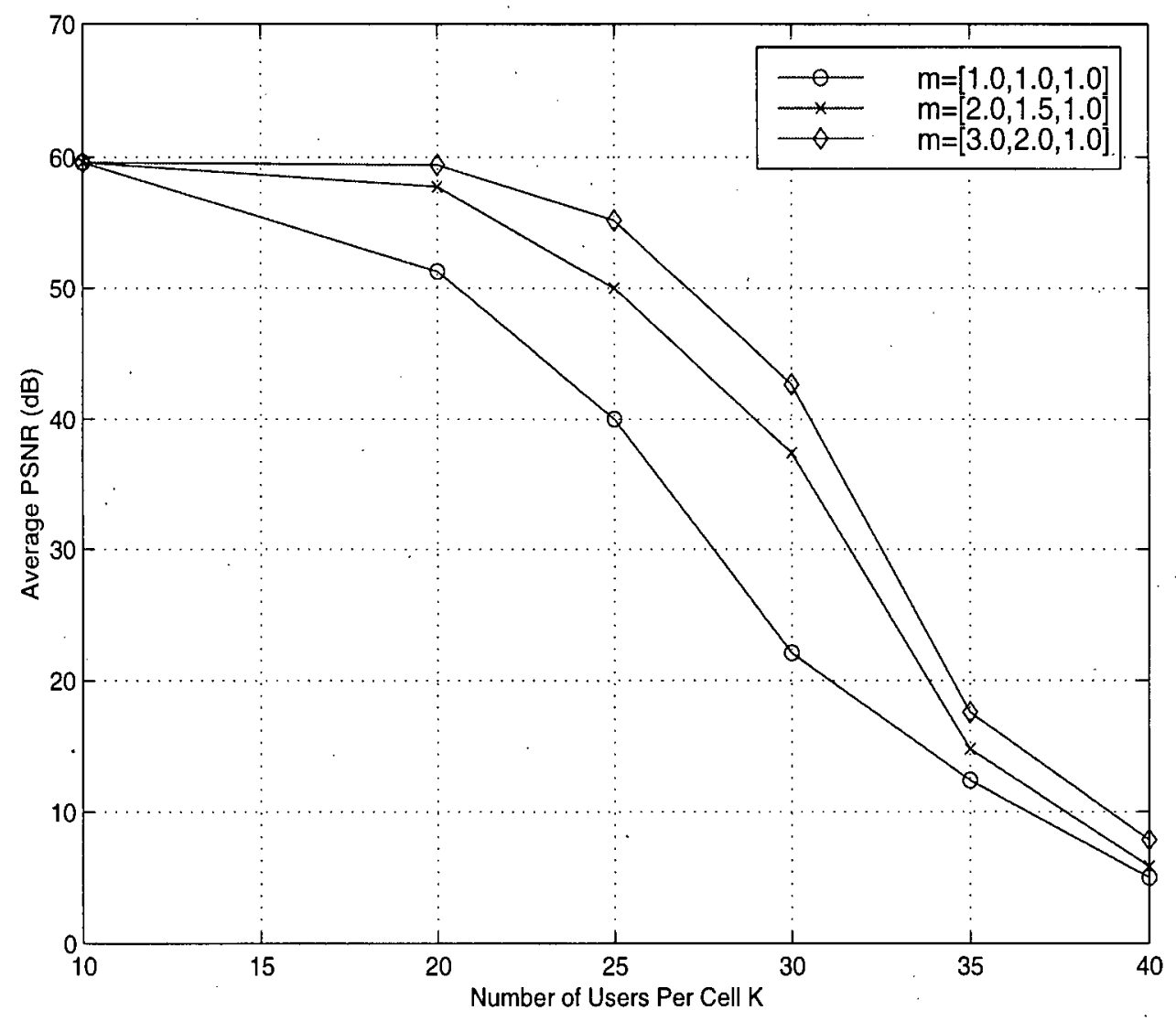

Fig. 5.4 PSNR Performance with Non-Identical $m$ Values for Each Multipath

channels modeled with non-identical set of $m$ values. As examples, we consider cases of $[\{3.0,2.0,1.0\}, \quad\{2.0,1.5,1.0\}, \quad\{1.0,1.0,1.0\}], \quad[\{2.0,1.5,1.0\}, \quad\{2.0,1.5,1.0\}$, $\{2.0,1.5,1.0\}]$ and $[\{2.0,1.5,1.0\},\{1.0,1.0,1.0\},\{1.0,1.0,1.0\}]$, respectively. For each case, the set of $m$ values in the first $\{\cdot\}$ correspond to the fading figures of the reference-cell channel, the set of $m$ values in the second $\{\cdot\}$ correspond to the fading figures of the 6 first-tier surrounding-cell channels, and the set of $m$ values in the third $\{\cdot\}$ correspond to the fading figures of the 12 second-tier surrounding-cell channels. The values in each $\{\cdot\}$ in turn represent the fading figures for the multipath signals in the order of arrivals. In the first case, we investigate 


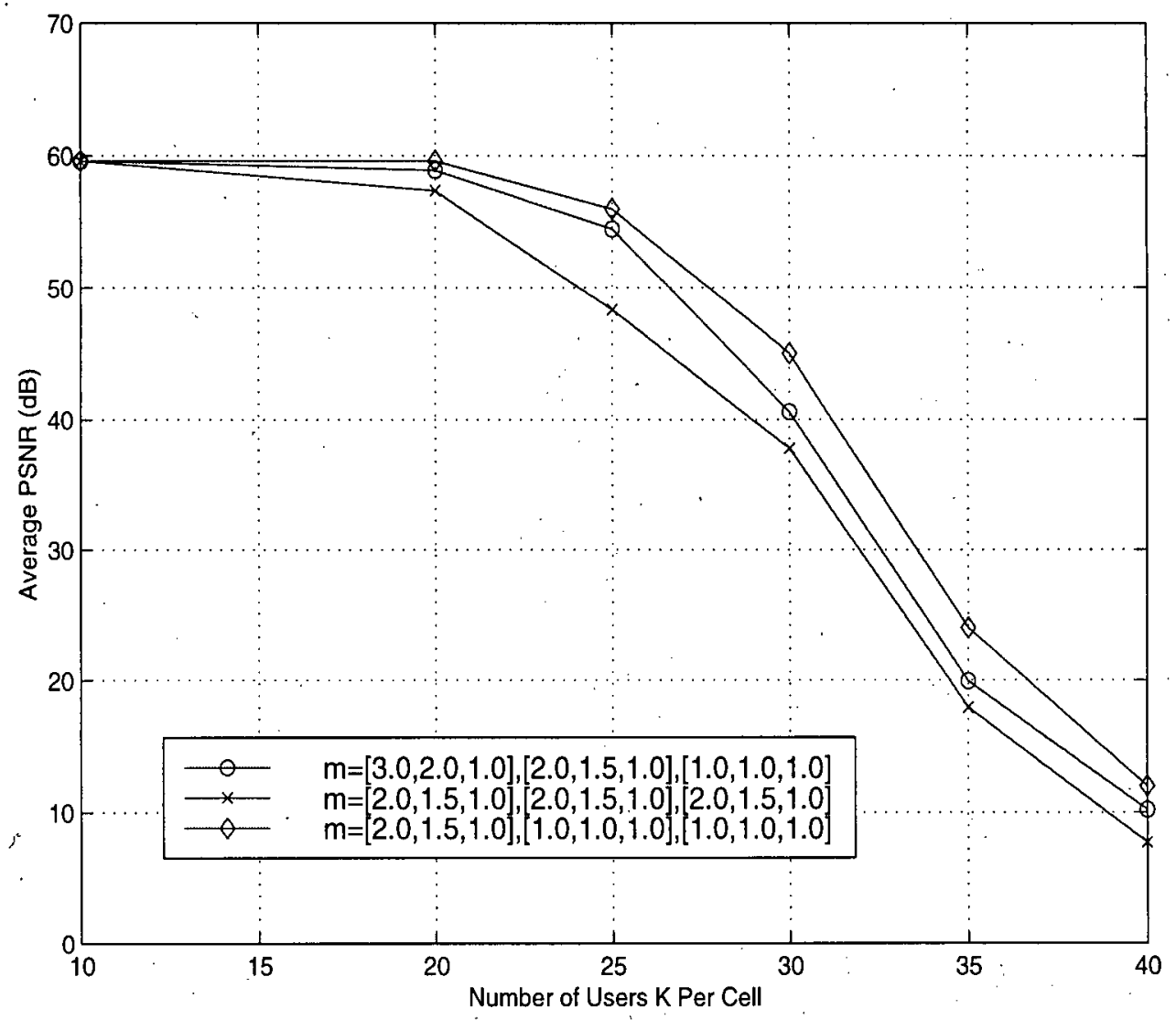

Fig. 5.5 PSNR Performance with Non-Identical $m$ Value Set for Channels from Different Tiers the system of which signals transmitted from the reference base station suffers relatively less severe fading than those from the surrounding base stations. The simulation resembles the physical situation in which the reference mobile is most likely closer to the reference base station than the other base stations. Thus, there is a higher probability for the existence of direct line-ofsight signals from the reference base station than from the surrounding base stations. As a result, it is justified to assume that the channel fading for the reference-cell signal is relatively less severe than the surrounding-cell signals. From the figure, we observe that the video performance of the first case is better than that of the second case where the reference cell has higher $m$ values or less 
fading. Next, for the transmission systems in the second and third case, we compare the PSNR performance where the reference-cell channels are assumed to share the same fading figure set but the surrounding-cell channels are assigned with different set of $m$ values. We observe that in the third case where the surrounding-cell channels exhibit more severe fading, better PSNR performance is obtained. This can be explained by the fact that the greater severity of fading in the surrounding-cell channels reduces the amount of multiple-access interference signals from reaching the reference mobile.

In Fig. 5.6, the effects of different user load of surrounding cells to video transmission performance are evaluated. In previous investigations, we have assumed that the number of users in the surrounding cells are the same as that of the reference cell. Here; we consider scenario of different surrounding-cell user loads as we evaluate the PSNR values of systems with all the 18 surrounding cells being occupied by $1 / 2,1 / 3$ and $1 / 4$ of the reference-cell user population.

As shown in Fig. 5.6, the PSNR performance improves as the surrounding-cell user load decreases. For example, at the capacity of 25 users in the reference cell, we observe an increase of about $14 \mathrm{~dB}$ and $19 \mathrm{~dB}$ in PSNR performance for the $1 / 3$ and $1 / 4$ capacity cases when compared to the $1 / 2$ capacity case, respectively. The gain in PSNR performance is due to the decrease in the amount of multiple-access interference generated from the neighboring-cell users. It has been clearly seen from this simulation that the user load of surrounding cells could have a significant impact on the video transmission performance in a CDMA system.

In Fig. 5.7, we investigate the CDMA video transmission performance with non-identical multipath intensity profile for channels of cells in different tiers . As examples, we consider cases of $\delta=0.2,0.2, \delta=0.2,0.4$ and $\delta=0.2,0.8$ where the first value corresponds to the logarithmic MIP exponent of the reference-cell channel, and the second value corresponds to that of the 


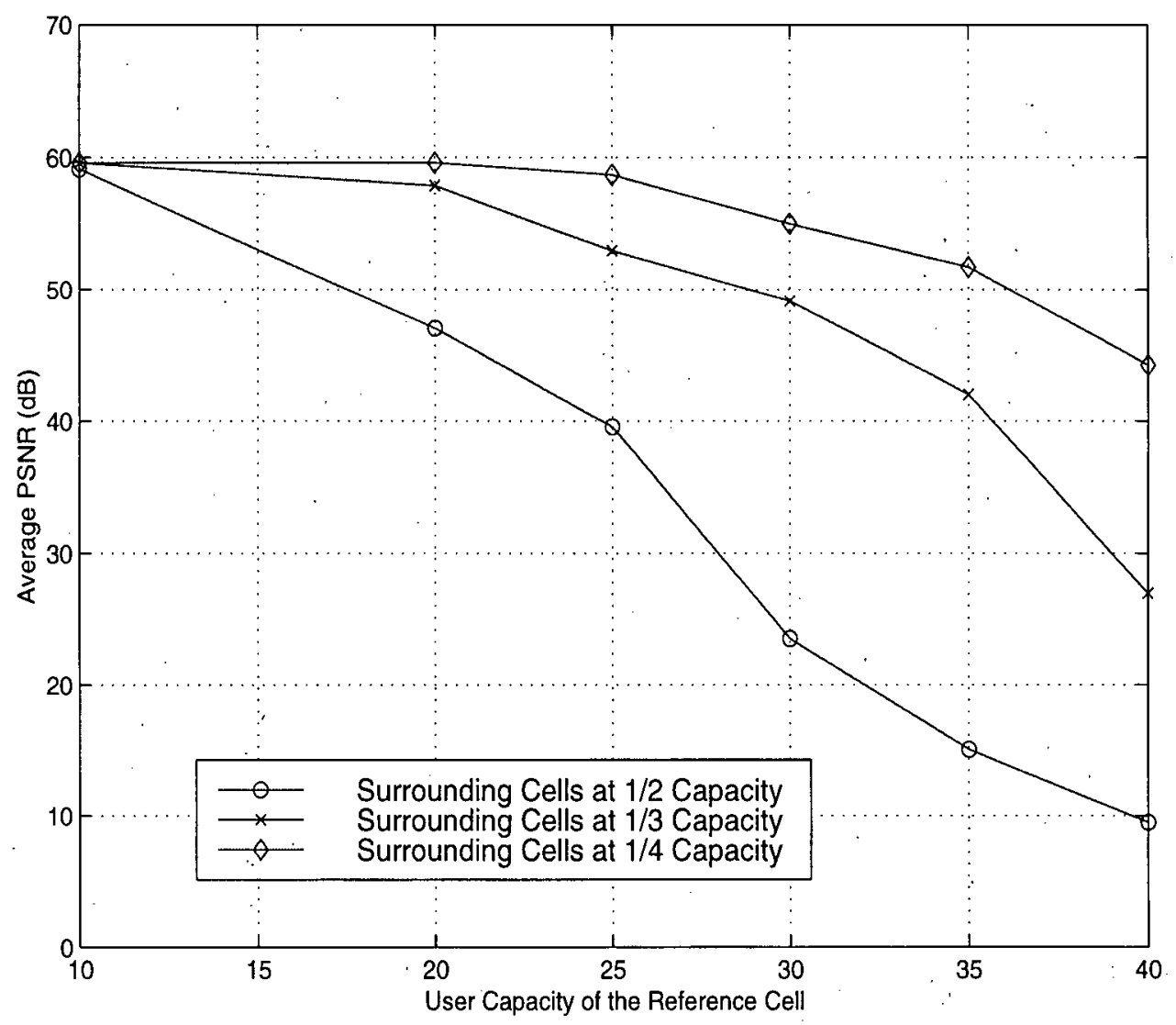

Fig. 5.6 PSNR Performance with Different Surrounding-Cell User Load

first and second tier surrounding-cell channels. Since signals transmitted from surrounding cells travel through different kinds of physical environment, it is reasonable to model the channels of the reference cell and surrounding cells with different $\delta$ values. As shown in the figure, there are significant differences in PSNR results between different cases of $\delta$ values. For instance, at the system capacity of 30 users per cell, we obtain PSNR improvement of approximately $10 \mathrm{~dB}$ and $20 \mathrm{~dB}$ in the second and third case (non-identical MIP) over the first case (uniform MIP), respectively. This can be explained by the fact that greater logarithmic MIP exponent in surrounding-cell signals means interferencing multipath signals are weaker when they reach the reference mobile, 


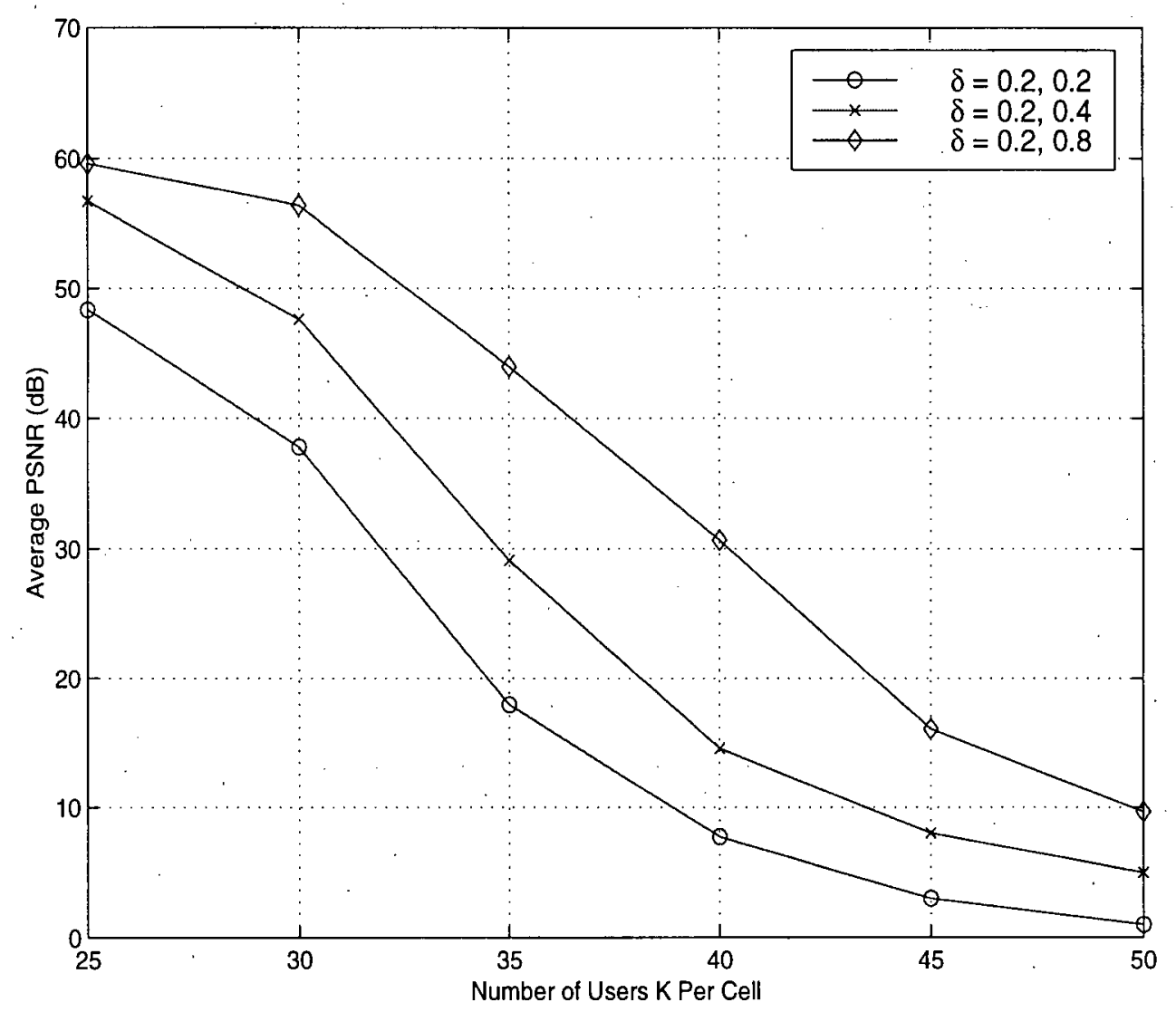

Fig. 5.7 PSNR Performance with Non-Identical MIP for Channels from Different Tiers which translates to less multiple-access interference to the received signal.

We have also investigated the impact of imperfect channel estimation of the channel tap weights on the video transmission quality. Similar to the single-cell scenario, we simulate the estimation errors as Gaussian random variables with mean of the exact fading amplitude and deviation variance denoted as $\sigma_{e}$. We have compared two cases including perfect channel estimation $\left(\sigma_{e}=0\right)$ as well as imperfect estimation with error variance $\sigma_{e}=0.25$ and the results are presented in Fig. 5.8. As seen from this figure, imperfect channel tap weight estimation could noticeably degrades PSNR performance. For example, at PSNR value of $40 \mathrm{~dB}$, the system 


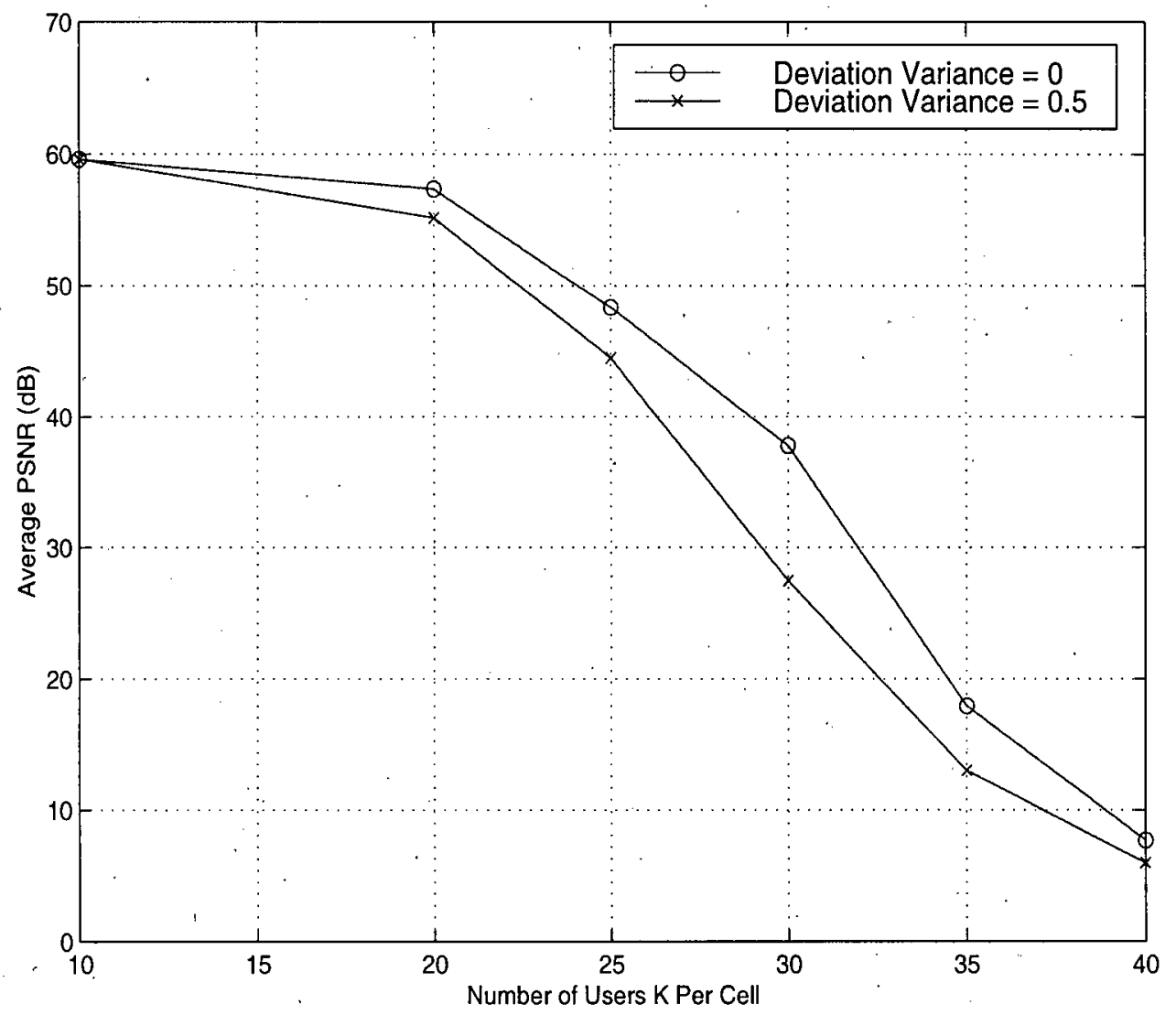

Fig. 5.8 PSNR Performance with Imperfect Channel Estimation capacity is reduced from 28 users to 23 users due the channel estimation errors.

Finally, we have evaluated the video transmission performance of the CDMA system with non-identical number of multipaths for cells at different tiers. In other investigations, we assume that all cells share the same number of multipaths; however, due to the variant nature of the physical environment that different base-station signals have to travel through, it is reasonable to assume that the number of multipaths for cells from different tiers are not necessarily identical. Moreover, it is also logical to assume that the neighboring-cell channels have longer delay spread or more multipaths due to the greater distance that the signals have to travel to reach the reference 


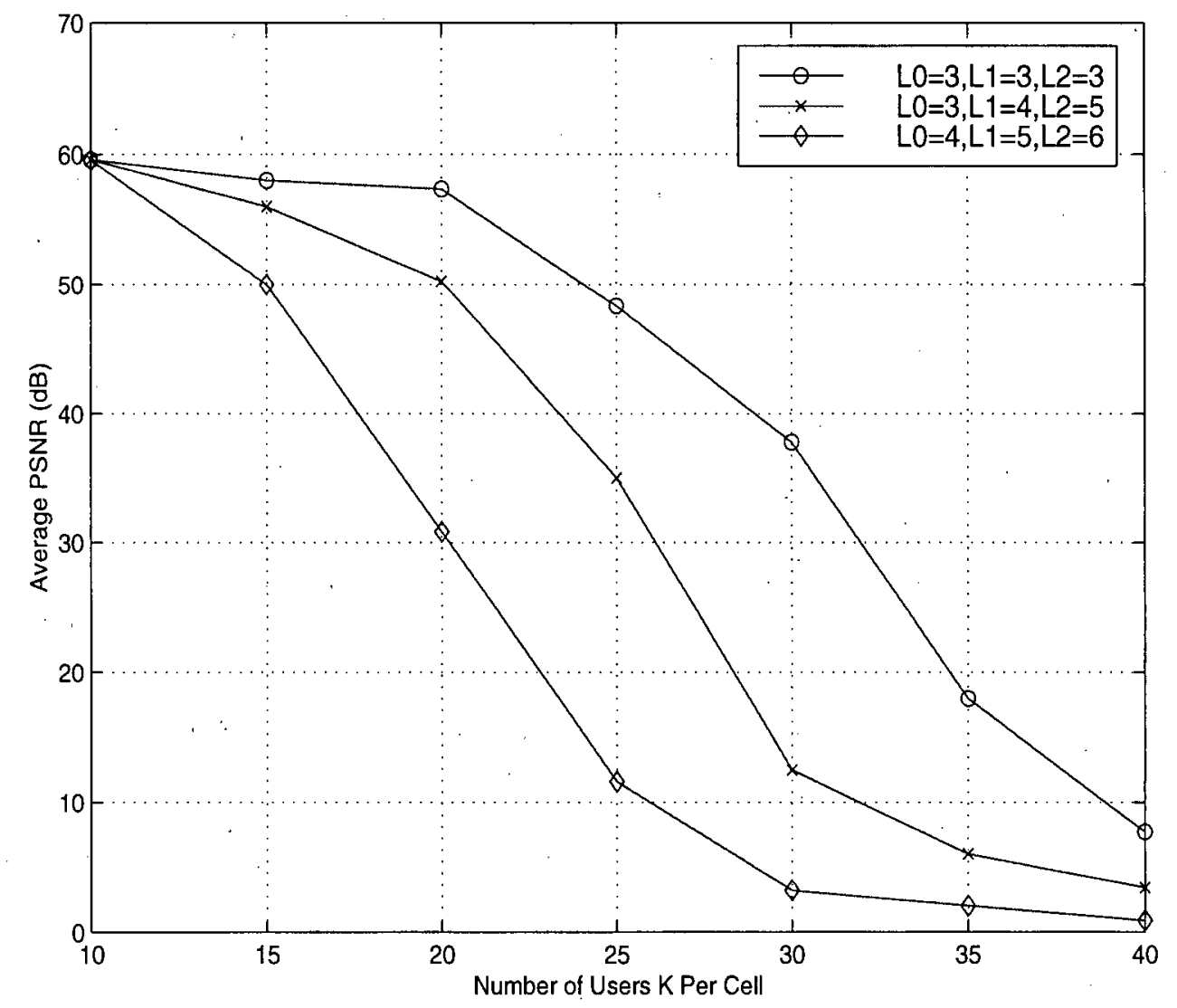

Fig. 5.9 PSNR Performance with Non-Identical Number of Multipaths for Different Tiers mobile. Typical performance evaluation results are presented in Fig. 5.9, where L0, L1 and L2 correspond to the multipath number of the reference cell, first-tier cells, and second-tier cells, respectively. We consider cases of $L 0=L 1=L 2=3, L 0=3, L 1=4, L 2=5$ as well as $L 0=4, L 1=5, L 2=6$. We observe a significant amount of PSNR degradation as the multipath number of the neighboring-cells increases, which is due to the corresponding increase of multiple-access interference from the extra multipath signals under consideration. 


\subsection{Conclusions}

In this chapter, we have investigated the video transmission performance of modified H.263 encoded data in a multiple-cell CDMA system over correlated Nakagami fading channels for the forward lịnk. The multiple-cell configuration model, channel model and cellular CDMA system model were explained in details. Mathematical analysis for the CDMA forward link was presented, and its results were compared with those obtained from computer simulation. It was found that Gaussian approximation provides better BER prediction for the multiple-cell forward link system than for the single-cell system. We have presented the PSNR performance of the integrated CDMA forward link video transceiver system through software simulation method. It was shown that video transmission performance improves as the average $m$ value of the multipath channel increases. Moreover, it was demonstrated that the more severe the surrounding-cell channel fading conditions, the better the PSNR performance can be achieved. It was found that neighboring-cell capacity has significant impact on video transmission quality of a cellular CDMA system. Further, we illustrated that the video performance improves for greater logarithmic MIP exponent for the outer-cell channels. Channel estimation errors have been shown to produce a significant degradation on system performance. Lastly, non-identical multipath channel modeling for cells at different tiers revealed the adverse effect of increased number of multipaths of neighboring-cell channels on the PSNR performance. 


\section{Chapter 6 CONCLUSIONS AND SUGGESTIONS FOR FUTURE RESEARCH}

\subsection{Conclusions}

In this thesis, we investigated the video transmission performance in the forward link of IS-95 based CDMA cellular systems over correlated Nakagami fading channels. The major contributions of the thesis are summarized as follows.

\subsubsection{A Modified H.263 Video Codec}

We have proposed a modified H.263 video codec to improve the average and frame-to-frame PSNR performance of the encoded video bitstream for transmissions in error-prone mobile channels: The proposed codec incorporates a selective forward error correction coding scheme and a periodic INTRA frame forced update mechanism to maximize video quality. This is achieved while avoiding excessive time delay critical for real-time video applications and minimizing channel coding redundancy as well as complexity. We have shown that improvement of $3-10 \cdot \mathrm{dB}$ in average PSNR performance can be achieved by the proposed scheme when compared to the original version.

\subsubsection{Correlated Nakagami Fading Simulator}

We have implemented in software a correlated Nakagami fading simulator to model the multipath mobile channels. The simulator is based on the physical model for the radio wave propagation process for different scattering environments. It generates fading output by the summation of complex phasors, with Doppler shift element incorporated to create the time correlation relationship between outputs. It has advantages over conventional Rayleigh and Rician fading simulation 
because Nakagami- $m$ distribution provides a more generalized and versatile fading modeling for the mobile channel. Moreover, the Doppler spectrum generated from this approach is the exact replica of the actual physical situation, as opposed to the idealistic U-tub shape obtained from conventional digital filtering method.

\subsubsection{Analysis of CDMA Forward Link}

We have presented the BER performance analysis of the CDMA forward link for both single-cell and multiple-cell environment employing the Gaussian approximation. It was observed that for random spreading sequences, the Gaussian approximation does not provide accurate predictions for BER performance of single-cell systems. On the other hand, it predicts the BER results quite accurately for multiple-cell systems. These observations are explained by the fact that in singlecell systems, the interference signals transmitted from the base station to the reference mobile exhibit the same amplitude fading, phase distortions and time delays, thus do not satisfy the random variable independency requirement of the central limit theorem. In the case of multiplecell environment, although the multiple-access interference from users within the same cell shares common channel characteristics, interference signals from different neighboring cells are statistically independent from each other. Therefore, the multiple-cell interference components are more accurately described by the Gaussian distribution as dictated by the Central Limit Theorem.

\subsubsection{End-to-end Video Transmission Performance Evaluation}

We have presented the PSNR performance of the modified H.263 coded video transmitted through the proposed IS-95 based CDMA system over correlated Nakagami fading channels, for both single-cell and multiple-cell environment. We have observed that the coherent RAKE receiver 
functions as a maximal-ratio combiner such that the optimal number of resolvable paths equals the total number of multipaths. In general, the PSNR performance improves as the $m$ values of the reference-cell channel increase due to the corresponding reduction in fading severity. The system also performs better for larger logarithmic MIP exponents due to the net gain in interference reduction versus desired signal power reduction. We also observed that the sensitivity of the video transmission performance on the channel estimation error can be significant. For multiple-cell systems, we found that better PSNR performance is achieved when the neighboring cells have more severe fading channel conditions, lower user capacity, and greater logarithmic MIP exponent. Lastly, we illustrated that increasing number of multipaths in surrounding-cell channels can degrade the video transmission performance in a CDMA system considerably.

\subsection{Suggestions for Future Research}

- One of the interesting topic for future research would be the evaluation of video transmission performance for the reverse link of the CDMA systems. This will complete the investigation of the performance of a two-way video communication system.

- Employing soft-decision Viterbi decoding at the receiver is also a worthwhile task for further research as it should improve the video transmission performance.

- By adopting the latest H.263+ compression standard for the video codec, new optional features from the standard can be employed to improve the video codec performance. 


\section{Glossary}

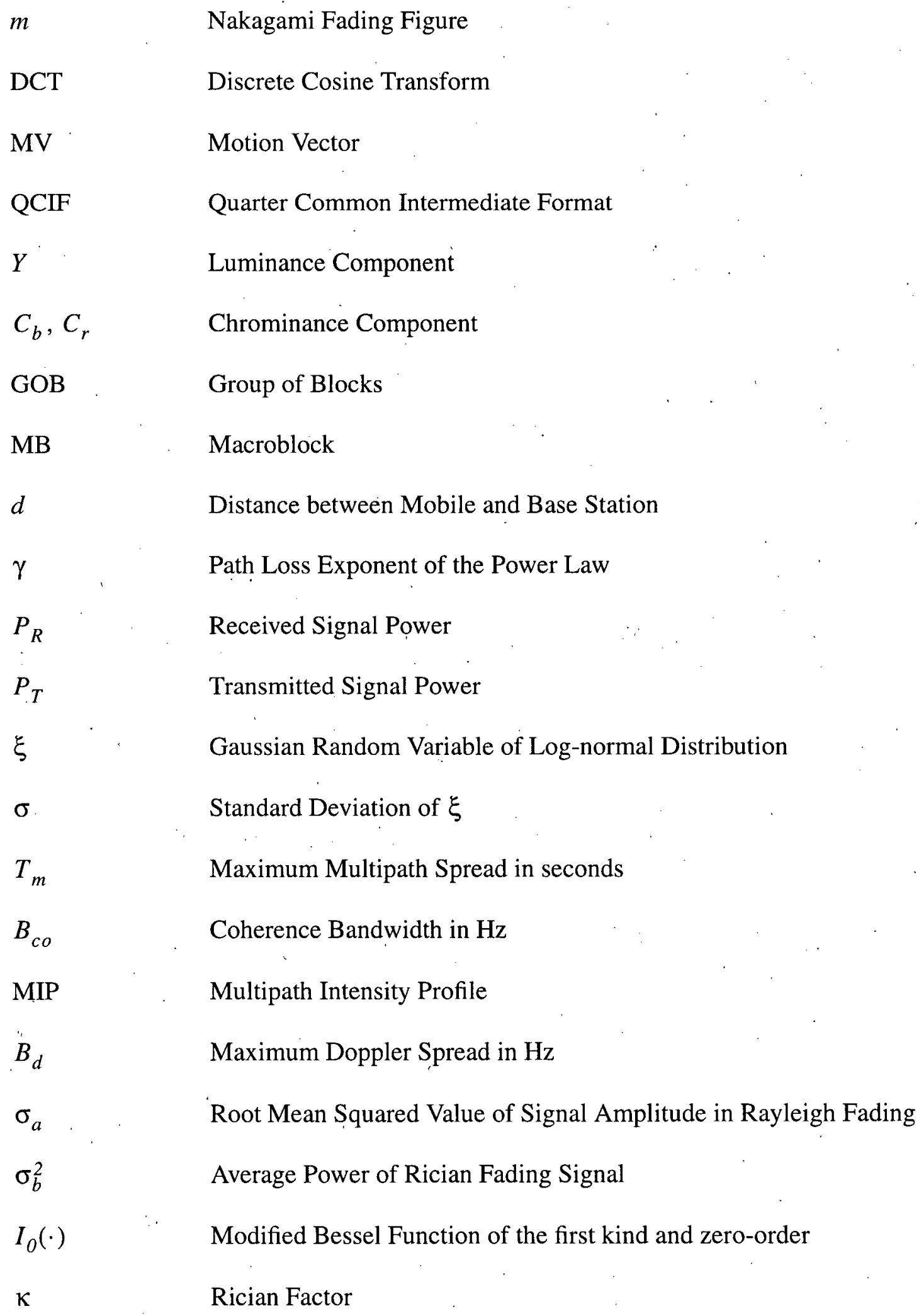


A

$\Gamma(m)$

$\Omega$

$B_{s s}$

$B$

PN

$Z_{M}$

$\alpha_{M}$

$Z^{\prime}$

$n$

$b_{i}$

$\phi(j)$

ARQ

FEC

AWGN

I-frame

P-frame

QCIF

PSNR

$o_{i}^{x}, r_{i}^{x}$

$P_{e}$

$\left\{c_{n}(t)\right\}$

$\left\{\beta_{n}(t)\right\}$
Peak Amplitude of the Dominant Signal

Gamma Function

Second moment of Nakagami Fading Signal Amplitude

Spread Spectrum Bandwidth in $\mathrm{Hz}$

Original Signal Bandwidth

Pseudo-noise

Output of the $M$ Correlator of a RAKE Receiver

RAKE Weight for the $M$ Correlator

Total Output of RAKE Correlators

Period of a Sequence

The $i$ th Binary Bit of a Sequence

Autocorrelation Function

Automatic Repeat Request

Forward Error Correction

Additive White Gaussian Noise

INTRA frame

Prediction Frame

Quadrature Common Intermediate Format

Peak Signal-to-Noise Ratio

Original and Reconstructed Pixel Components

Bit Error Probability

Frequency-Selective Channel Complex Tap Weights

Channel Tap Weight Amplitude 


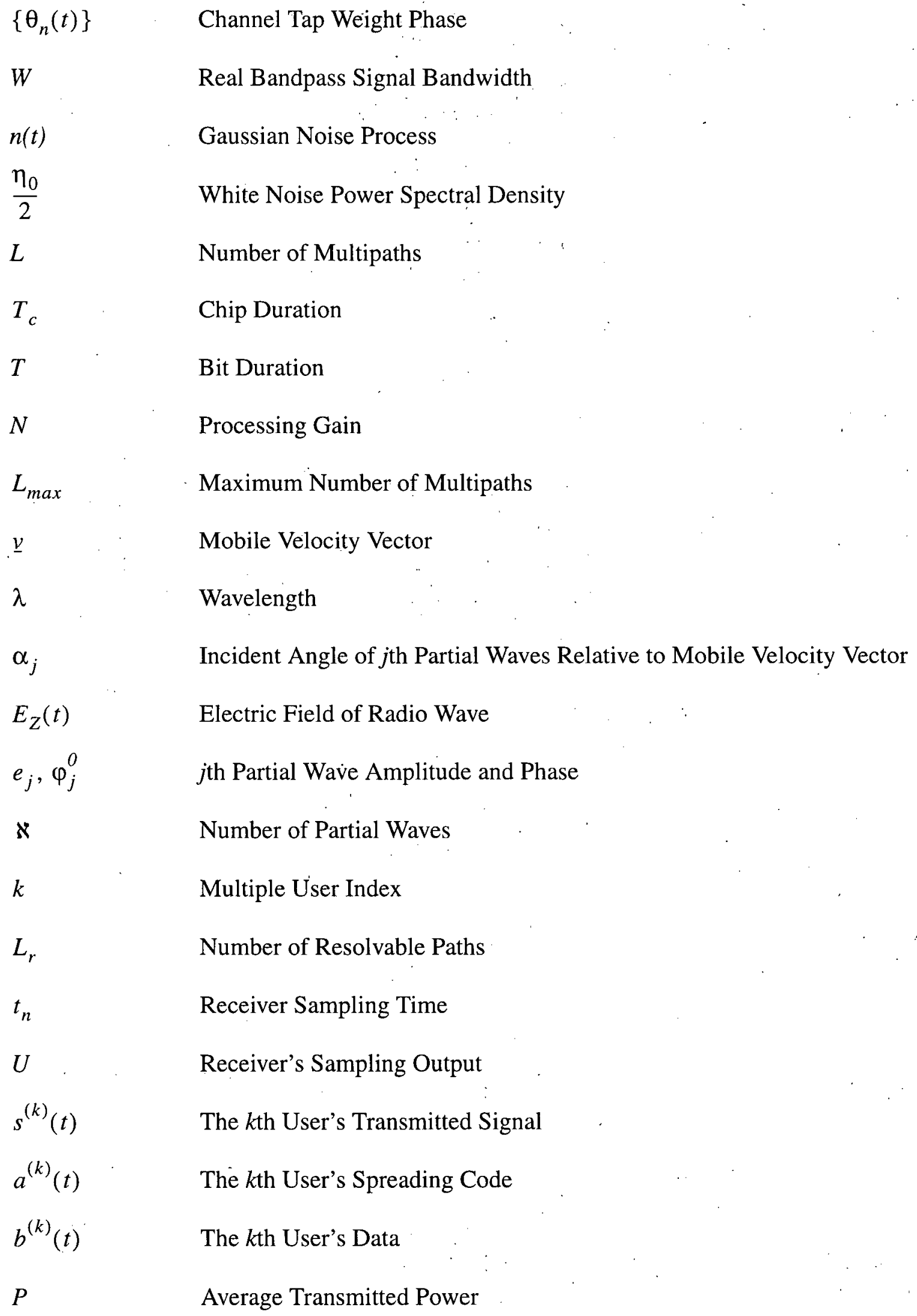


Common Carrier Frequency

Common Phase of the Modulator

$p_{a}(t)$

Rectangular Pulses of Chips

$p_{b}(t)$

Rectangular Pulses of Data Bits

$r(t)$

Received Signal After the Channel

K

Total number of users

$\varphi_{l}$

Phase of the $l$ th Path Signal

$\tau_{l}$

Multipath Time Delay of the $l$ th Path

$S^{(n)}$

Desired Signal Amplitude at the $n$th Finger

$I_{m a i}^{(n)}$

Multiple-Access Interference at the $n$th Finger

$I_{s i}^{(n)}$

Self-Noise Interference at the $n$th Finger

$I_{n i}^{(n)}$

White Noise Interference Term at the $n$th Finger

$b_{0}^{(k)}$

Information Bit of the $k$ th User to be Detected

$b_{-1}^{(k)}$

Precedent Information Bit of the $k$ th User

Multipath Time Delay Difference Between the $n$th and $l$ th Paths

$\varphi_{n l}$

Multipath Phase Difference Between the $n$th and $l$ th Paths

$R_{k 0}[\tau], \hat{R}_{k 0}[\tau]$

Continuous-Time Partial Cross-Correlation Functions Between the kth and the Reference User

$C_{k 0}$

Discrete Aperiodic Cross-Correlation Functions Between the kth and the Reference User

$\sigma_{S}^{2}$

Variance Sum of All Interference Terms

$\sigma_{M A I, n}^{2}$

Conditional Variance of Multiple-Access Interference at the $n$th Finger

$\dot{\sigma}_{S I, n}^{2}$

Conditional Variance of Self-Noise Interference at the $n$th Finger

$\sigma_{N I, n}^{2}$

Conditional Variance of White Noise at the $n$th Finger 
Average Cross-Correlation Parameter

$\Omega_{l}$

$U_{s}$

$E_{b}$

$\delta$

$S$

$\Upsilon$

$\Gamma(a, x)$

$\hat{P}_{q}$

$P_{q}$

$d_{q}$

$\zeta_{q}, \sigma_{q}$

$s_{q}^{(k)}(t)$

$a_{q}^{(k)}(t)$

$b_{q}^{(k)}(t)$

$P_{q}, \hat{P_{q}}$

$\phi_{q}$

$K_{q}$

$\hat{r}(t)$

$Q$

$L_{q}$

$\varphi_{q l}$

$\overline{P_{e}^{\prime}}$
Average Signal Power of the $l$ th Path

Desired Signal Component of the Receiver Response

Signal Energy Per Bit

Exponent of Logarithmic Multipath Intensity Profile

Random Component of the SNR

Deterministic Component of the SNR

Incomplete Gamma Function

Average Received Power from the $q$ th Base Station

Transmitted Signal Power from the $q$ th Base Station

Distance Between the Mobile and the $q$ th Base Station

Gaussian Random Variable and its Standard Deviation for the Signal Path from the $q$ th Base Station

The $k$ th User's Transmitted Signal of the qth Cell

The $k$ th User's Spreading Code of the $q$ th Cell

The $k$ th User's Data of the $q$ th Cell

Transmitted and Received Power of Signal from the $q$ th Cell .

Carrier Phase of Signal from the $q$ th Cell

Number of Users in the $q$ th Cell

Received Signal After the Channel for Multiple-Cell Environment

Total Number of Cells

Number of Multipaths of Signal from the $q$ th Cell

Phase of the $l$ th Path from the $q$ th Cell

Average-Averaged Probability of Error for Multiple-Cell 
$\theta_{q l}$

$\tau_{q l}$

$\beta_{q l}$

$\hat{U}$

$\hat{S}^{(n)}$

$\hat{I}_{m a i}^{(n)}$

$\hat{I}_{s i}^{(n)}$

$\hat{I}_{n i}^{(n)}$

$\rho_{q}$

$\hat{U}_{s}$

$\hat{\sigma}_{S}^{2}$

$\hat{\sigma}_{M A I, n}^{2}$

$\hat{\sigma}_{S I, n}^{2}$

$\hat{\sigma}_{N I, n}^{2}$

$\Omega_{q l}$

$\delta_{q}$

$S_{m}$

$\Upsilon_{m}$
Channel Phase Shift for the $l$ th Path from the $q$ th Cell

Multipath Time Delay for the $l$ th Path from the $q$ th Cell

Fading Amplitude for the $l$ th Path from the $q$ th Cell

Receiver Response at Sampling Time for Multiple-Cell

Desired Signal Amplitude at the $n$th Finger for Multiple-Cell

Multiple-Access Interference at the $n$th Finger for Multiple-Cell

Self-Noise Interference at the $n$th Finger for Multiple-Cell

White Noise Interference Term at the $n$th Finger for Multiple-Cell

Large-Scale Fading Attenuation Factor

Desired Signal Component of the Receiver Response for Multiple-Cell

Variance Sum of All Interference Terms for Multiple-Cell

Conditional Variance of Multiple-Access Interference at the $n$th Finger for Multiple-Cell

Conditional Variance of Self-Noise Interference at the $n$th Finger for Multiple-Cell

Conditional Variance of White Noise at the $n$th Finger for MultipleCell

Average Signal Power of the $l$ th Path from the $q$ th Cell

Decay Exponent for the $q$ th Cell's MIP

Random Component of the SNR for Multiple-Cell

Deterministic Component of the SNR for Multiple-Cell 


\section{Bibliography}

[1] R. Kohno, R. Meidan and L. B. Milstein, "Spectrum spectrum access methods for wireless communications," IEEE Communications Magazine, pp. 58-67, Jan. 1995.

[2] D. L. Schilling, R. L. Pickholtz, and L. B. Milstein, "Spread spectrum goes commercial," IEEE Spectrum, pp. 40-45, Aug. 1990.

[3] R. L. Pickholtz, L. B. Milstein, and D. L. Schilling, "Spread spectrum for mobile communications," IEEE Trans. Vehicular Tech., VT-40, pp. 313-322, May 1991.

[4] A. Fukasawa, T. Sato, Y. Takizawa, T. Kato, M. Kawebe and R. E. Fisher, "Wideband CDMA system for personal radio communications," IEEE Communications Magazine, Oct. 1996.

[5] T. S. Rappaport, Wireless Communications Principles \& Practice. New Jersey: Prentice Hall, 1996.

[6] R. Price and P. E. Green, "A communication technique for multipath channel," Proceedings of the IRE, pp. 555-570, Mar. 1958.

[7] W. C. Y. Lee, "Overview of cellular CDMA," IEEE Trans. Vehicular Tech., vol. 40, pp. 291-302, May 1991.

[8] D. C. Cox, R. Murray and A. Norris, "800 MHz attenuation measured in and around suburban houses," AT\&T Bell Laboratory Tech. Journal, vol. 673, no. 6, July-Aug. 1984.

[9] EIA/IS-95, "Dual mode mobile station-base station wideband spread spectrum compatibility standard," PN-3119, Electronics Industries Association, Engineering Department, Dec. 1992.

[10] N. Morinaga, M. Nakagawa and R. Kohno, "New concepts and technologies for achieving highly repliable and high-capacity multimedia wireless communications systems", IEEE Communications Magazine, pp. 34-40, Jan. 1997.

[11] P. Bahl and B. Girod, "Wireless video," IEEE Communications Magazine, June 1998.

[12] ITU Telecom. Standardization Sector of ITU, "Video coding for low bitrate communication," ITU-T Recommendation H.263, Mar. 1996. 
[13] M. Khansari, A. Jalali, E. Dubois and P. Mermelstein, "Low bit-rate video transmission over fading channels for wireless microcellular systems," IEEE Trans. on Circuits Syst. Video Technol., vol. 6, no. 1, Feb. 1996.

[14] R. Stedman, H. Gharavi, L. Hanzo and R. Steele, "Transmission of subband-coded images via mobile channels," IEEE Trans. Circuits Syst. Video Technol., vol. 3, no. 1, Feb. 1993.

[15] R. Talluri, "Error-resilient video coding in the ISO MPEG-4 standard," IEEE Communications Magazine, June 1998.

[16] M. Khansari, A. Zakauddin, W. Y. Chan, E. Dubois and P. Mermelstein, "Approaches to layered coding for dual-rate wireless video transmission," Proc. of Int. Conf. on Image Processing, 1994.

[17] N. Farber and B. Girod, "Robust H.263 compatible video transmission for mobile access to video servers," Proc. of Int. Conf. on Image Processing, 1997.

[18] L. Hanzo and J. Streit, "Adaptive low-rate wireless videophone schemes," IEEE Trans. Circuits Syst. Video Technol., vol. 5, no. 4, Aug. 1995.

[19] J. Streit and L. Hanzo, "Quadtree-based reconfigurable cordless videophone systems," IEEE Trans. Circuits Syst. Video Technol., vol. 6, no. 2, Apr. 1996.

[20] P. Cherriman and L. Hanzo, "Programmable H.263-based wireless video transceivers for interference-limited environments," IEEE Trans. Circuits Syst. Video Technol., vol. 8, no. 3, June 1998.

[21] H. Liu and M. E. Zarki, "Transmission of video telephony images over wireless channels," Wireless Networks, vol. 2, no. 3, 1996.

[22] . H. Suzuki, "A statistical model for urban radio propagation," IEEE Trans. on Comm., vol. COM-25, pp. 673-680, July 1977.

[23] T. Eng and L. B. Milstein, "Coherent DS-CDMA performance in Nakagami multipath fading," IEEE Trans. on Comm., vol. 43, no. 2/3/4/, February/March/March/April 1995.

[24] W. Braun and U. Dersch, "A physical mobile radio channel model," IEEE Trans. Veh. Technol., vol. 40, no. 2, pp. 472-482, 1991. 
[25] ITU-T Recommendation 601, Encoding Parameters of Digital Television for Studios, 1982

[26] J. G. Proakis, Digital Communications. New York: McGraw Hill, 1983.

[27] M. Yokoyama, "BPSK system with sounder to combat Rayleigh fading in mobile radio communication," IEEE Trans. Veh. Tech., vol. VT-34, pp. 35-40, Feb. 1985.

[28] F. Davarian, "Mobile digital communication via tone calibration," IEEE Trans. Veh. Tech., vol. VT-36, pp. 55-62, May 1987.

[29] G. P. Efthymoglou, V. A. Aalo and H. Helmken, "Performance Analysis of coherent DSCDMA Systems in a Nakagami fading channel with arbitrary parameters," IEEE Trans. Veh. Tech., vol. 46, no. 2, May 1997.

[30] G. L. Turin, "Introduction to spread-spectrum antimultipath techniques and their application to urban digital radio," Proc. IEEE, vol. 68, pp.328-353, Mar. 1980.

[31] M. B. Pursley, "Performance evaluation for phase-coded spread-spectrum multiple-access communication - Part I: System Analysis," IEEE Trans. on Comm., vol. COM-25, no. 8, July 1977.

[32] M. H. Fong, V. K. Bhargava and Q. Wang, "Concatenated orthogonal/PN spreading sequences and their application to cellular DS-CDMA systems with integrated traffic," IEEE J. Select. Areas Commun., vol. 14, no. 3, Apr. 1996.

[33] M. H. Fong, Q. Wang and V. K. Bhargava, "Concatenated orthogonal/PN codes for DSCDMA systems in a multi-user and multipath fading environment," Proc. of Global Telecomm. Conf., 1994.

[34] D. V. Sarwate and M. B. Pursley, "Crosscorrelation properties of pseudorandom and related sequences," Proc. IEEE, vol. 68, pp. 593-619, May 1980.

[35] M. Nakagami, "The m-distribution - a general formula of intensity distribution of rapid fading," in Statistical Methods in Radio Wave Propagation, W. G. Hoffman, Ed. Oxford, England: Pergamon, pp. 3-36, 1960.

[36] S. A. Abbas and A. U. Sheikh, "A geometric theory of Nakagami fading multipath mobile radio channel with physical interpretations," Proc. Vehecular Tech. Conf., 1996. 
[37] W. R. Braun and U. Dersch, "A physical mobile radio channel model," IEEE Trans. Veh. Tech., vol. 40, no. 2, May 1991.

[38] G. Turin, F. Clapp, T. Johnston, S. Fine and D. Lavry, "A statistical model of urban multipath propagation," IEEE Trans. Veh. Tech., vol. VT-21, no. 1, pp. 1-9, 1972.

[39] M. V. Clark, L. J. Greenstein, W. K. Kennedy and M. Shafi, "Matched filter performance bounds for diversity combining receivers in digital mobile radio," IEEE Trans. Veh. Tech., vol. 41, no. 4, pp. 356-362, 1992.

[40] H. Xiang, "Binary code-division multiple-access systems operating in multipath fading, noisy channels," IEEE Trans. Comm., vol. COMM-33, pp. 775-784, Aug. 1985.

[41] W. C. Jakes, Ed., Microwave Mobile Communications. New Jersey: IEEE Press, 1993.

[42] E. Geraniotis, "Direct-sequence spread-spectrum multiple-access communications over nonselective and frequency-selective rician fading channels," IEEE Trans. on Comm., vol. COM-34, pp. 756-764, Aug. 1986.

[43] E. A. Geraniotis and M. B. Pursley, "Performance of coherent direct-sequence spreadspectrum communications over specular multipath fading channels," IEEE Trans. on Comm., vol. COM-33, pp. 502-508, June 1985.

[44] K. Yao, "Error probability of asynchronous spread spectrum multiple access communication systems," IEEE Trans. on Comm., vol. COM-25, pp. 803-809, Aug. 1977.

[45] A. Andreadis, G. Benelli, A. Garzelli and S. Susini, "FEC coding for H.263 compatible video transmission," Proc. of Int. Conf. on Image Processing, 1997.

[46] E. Steinbach, N. Faerber, B. Girod, "Standard compatible extension of H.263 for robust video transmission in mobile environments", IEEE Trans. on Circuits Syst. Video Technol., vol. 7, pp. 872-881, Dec. 1997.

[47] . H. Ibaraki et al., "Mobile video communication techniques and services", Proc. of SPIE Conf. on Visual Commun. and Image Proc., pp. 1029-1033, 1995.

[48] U. Horn, B. Girod and B. Belzer, "Scalable video coding with multiscale motion compensation and unequal error protection", Proc. Int. Symp. on Multimedia Commun. and Video Coding, Oct. 1995. 
[49] T. Yang, J. Chalidabhongse and C. C. J. Kuo, "Performance study of robust wireless video transmission over fading channels," Conf. Rec. of the 30th Asilomar Conf. on Signals, Systems and Computers, 1997.

[50] P. Bahl and I. Chlamtac, "H.263 based video codec for real-time visual communications over wireless radio networks," Proc. of Int. Conf. on Universal Personal Comm., 1997.

[51] U. Dersch and R. J. Ruegg, "Simulations of the time and frequency selective outdoor mobile radio channel," IEEE Trans. Veh. Tech., vol. 42, no. 3, Aug. 1993.

[52] A. H. Wojnar, "Unknown bounds on performance in Nakagami channels," IEEE Trans. Commun. vol. COMM-34, pp. 22-24, Jan. 1986.

[53] K. S. Gilhousen, I. M. Jacobs, R. Padovani, A. J. Viterbi, L. A. Weaver, Jr., and C. E. Wheatley III, " On the capacity of a cellular CDMA system," IEEE Trans. Veh. Tech., vol. 40, no. 2, May 1991.

[54] K. R. Rao and J. J. Hwang, Techniques and Standards for Image, Video, and Audio Coding, New Jersey: Prentice Hall PTR, 1996.

[55] W. C. Y. Lee, Mobile Cellular Telecommunications: Analog and Digital Systems, McGraw-Hill International Editions, 1995.

[56] A. Salmasi and K. S. Gilhousen, "On the system design aspects of code division multiple access (CDMA) applied to digital cellular and personal communications networks," Proc. of Veh. Tech. Conf., 1991.

[57] R. L. Peterson, R. E. Ziemer and D. E. Borth, Introduction to Spread Sectrum Communications, New Jersey: Prentice Hall, 1995.

[58] F. Davarian, "Channel simulation to facilitate mobile-satellite communications research," IEEE Trans. Commun. vol. COMM-35, no. 1, Jan. 1987. 


\section{Appendix A. Derivation of Multiple-Access Interference Variance for the Gaussian Approximation}

From (4.9b), the multiple-access interference component in the output response of the receiver is

$$
I_{m a i}^{(n)}=\sqrt{\frac{P}{2}} \cdot \sum_{k=1}^{K-1} \sum_{l=0}^{(k)} \beta_{n} \beta_{l} \cdot\left\{b_{-1}^{(k)} R_{k 1}\left(\tau_{n l}\right)+b_{0}^{(k)} \hat{R}_{k 1}\left(\tau_{n l}\right)\right\} \cdot \cos \left(\varphi_{n l}\right)
$$

Assuming that the period of the CDMA spreading code sequence (see Eq. (4.5)) is large as compared to the processing gain $N=T / T_{c}$, we can model the sequences as random binary sequences, which are mutually independent. As such, $I_{m a i}^{(n)}$ can be treated as a summation of independent random variables. In light of the presence of the cosine term, each summation component has zero mean. Thus, its variance conditioned on $\beta_{n}$ becomes $\sigma_{m a i}^{(n)}=E\left[\left(I_{m a i}^{(n)}\right)^{2}\right]$ which can be expressed as

$$
\sigma_{M A I}^{(n)}=E\left[\left(\sqrt{\frac{P}{2}} \cdot \sum_{k=1}^{K-1} \sum_{l=0}^{(k)} \beta_{n} \beta_{l} \cdot\left\{b_{-1}^{(k)} R_{k 1}\left(\tau_{n l}\right)+b_{0}^{(k)} \hat{R}_{k 1}\left(\tau_{n l}\right)\right\} \cdot \cos \left(\varphi_{n l}\right)\right)^{2}\right]
$$

From (A2), assuming same number of multipaths for every user (i.e., $L^{(k)}=L$ ) and since $P$ and $\beta_{n}$ are considered as constant, we have

$$
\sigma_{M A I}^{(n)}=\frac{P}{2} \cdot \sum_{k=1}^{K-1} \sum_{l=0}^{L-1}\left\{\beta_{n}\right\}^{2} E\left[\left\{\beta_{l}\right\}^{2}\right] E\left[\left\{b_{-1}^{(k)} R_{k 1}\left(\tau_{n l}\right)+b_{0}^{(k)} \hat{R}_{k 1}\left(\tau_{n l}\right)\right\}^{2}\right] E\left[\cos ^{2}\left(\varphi_{n l}\right)\right]
$$

Since $E\left[\cos ^{2}\left(\varphi_{n l}\right)\right]=1 / 2$ and by assuming that $b_{-1}^{(k)}$ and $b_{0}^{(k)}$ are i.i.d. random variables with equal probability taking values from the alphabet $\{ \pm 1\}$, we have

$$
\begin{aligned}
E\left[\left\{b_{-1}^{(k)} R_{k 1}\left(\tau_{n l}\right)+b_{0}^{(k)} \hat{R}_{k 1}\left(\tau_{n l}\right)\right\}^{2}\right] & =E\left[R_{k 1}^{2}\left(\tau_{n l}\right)+\hat{R}_{k 1}^{2}\left(\tau_{n l}\right)\right] \\
& =\frac{1}{T} \cdot \int_{0}^{T} R_{k 1}^{2}(\tau)+\hat{R}_{k 1}^{2}(\tau) d \tau
\end{aligned}
$$


The above integral can be evaluated by using the results in [31] to give

$$
\begin{aligned}
E\left[R_{k 1}^{2}\left(\tau_{n l}\right)+\hat{R}_{k 1}^{2}\left(\tau_{n l}\right)\right] & =\frac{T^{2}}{3 N^{3}} \cdot E\left[r_{k 1}^{\prime}(N)\right] \\
& =\frac{T^{2}}{3 N^{3}} \cdot r_{K}(N)
\end{aligned}
$$

where

$$
\begin{aligned}
r_{k i}^{\prime}(N)= & \sum_{l=0}^{N-1}\left\{C_{k i}^{2}(l-N)+C_{k i}(l-N) C_{k i}(l-N+1)+C_{k i}^{2}(l-N+1)\right. \\
& \left.+C_{k i}^{2}(l)+C_{k i}(l) C_{k i}(l+1)+C_{k i}^{2}(l+1)\right\} .
\end{aligned}
$$

Substituting (A5) into (A3), we obtain the expression shown in (4.17):

$$
\begin{aligned}
\sigma_{M A I}^{(n)} & =\frac{P}{2} \cdot \sum_{k=1}^{K-1} \sum_{l=0}^{L-1}\left\{\beta_{n}\right\}^{2} \cdot E\left[\left\{\beta_{l}\right\}^{2}\right] \cdot \frac{T^{2}}{3 N^{3}} \cdot r_{K}(N) \cdot \frac{1}{2} \\
& =\frac{P}{4} \cdot\left\{\beta_{n}\right\}^{2} \cdot \sum_{k=1}^{K-1} \frac{T^{2}}{3 N^{3}} r_{K}(N) \sum_{l=0}^{L-1} E\left[\left\{\beta_{l}\right\}^{2}\right] .
\end{aligned}
$$




\section{Appendix B. Evaluation of the Mean of Attenuation Factor $\rho_{q}$}

The evaluation of the mean of attenuation factor $\rho_{q}$ is based on the cell model illustrated in Fig. 5.2. In order to simplfy the mathematical analysis, the cells are assumed to be circular and uniform in size. To reiterate, in (5.16) we have defined

$$
\rho_{q}=\left(\frac{d_{0}}{d_{q}}\right)^{\gamma} 10^{\left(\zeta_{0}-\zeta_{q}\right) / 10}
$$

In (B1), $d_{q}$ is the distance between the reference mobile to the $q$ th base station, which is a random variable dependent on the reference mobile location. $\gamma$ is the path loss exponent, and $\zeta_{q}$ is a Gaussian random variable with zero mean and standard variance $\sigma_{q}$.

The mobile location is a function of both the distance and the angle with respect to the reference base station as shown in Fig. 5.2. It is assumed that the reference mobile are equally likely to be located anywhere within the reference cell. Let the random distance between the mobile and reference base station be $d_{0}=R$. Then, the PDF of $R$ for a circular cell is a function directly proportional to the circle circumference and can be written as

$$
p_{R}(r)=k r, \quad 0 \leq r \leq \mathfrak{R}
$$

where $k$ is a constant and $\Re$ is the cell radius. By definition, $\int_{0}^{\Re} p_{R}(r) d r=1$, thus we have

$$
p_{R}(r)=\frac{2 r}{\Re^{2}}
$$

The PDF of the angle $\theta$ is a uniform distribution over $[0,2 \pi)$, i.e.

$$
p(\dot{\theta})=\frac{1}{2 \pi}
$$

Since the attenuation factor $\rho_{q}$ is composed of random variables $r$ and $\theta$, we have, 


$$
E\left[\rho_{q}\right]=\int_{0}^{2 \pi R} \int_{0} p(r) p(\theta)\left(\frac{r}{d_{q}}\right)^{\gamma} E\left[10^{\left(\zeta_{0}-\zeta_{q}\right) / 10}\right] d r d \theta
$$

which, after substitutions of $p(r)$ and $p(\theta)$, becomes

$$
E\left[\rho_{q}\right]=\frac{1}{\pi \Re^{2}} \int_{0}^{2 \pi R} \int_{0}\left(\frac{r}{d_{q}}\right)^{\gamma} E\left[10^{\left(\zeta_{0}-\zeta_{q}\right) / 10}\right] r d r d \theta
$$

From [53], we have

$$
E\left[10^{\left(\zeta_{0}-\zeta_{q}\right) / 10}\right]=\exp \left[\left(\frac{\sigma \ln 10}{10}\right)^{2}\right] \cdot\left\{1-Q\left[\frac{10 \gamma}{\sqrt{2 \sigma^{2}}} \log \left(\left(\frac{d_{q}}{d_{0}}\right)-\frac{\sqrt{2 \sigma^{2}}}{10} \ln 10\right)\right]\right\}
$$

By applying cosine law to the geometry of cell configuration illustrated in Fig. 5.2, we obtain $d_{q}$ for the 6 first-tier cells, the 6 farther second-tier cells, and the 6 closer second-tier cells, respectively, as follow:

$$
\begin{gathered}
d_{q}=\sqrt{(\sqrt{3} R)^{2}+d_{0}^{2}+2 \sqrt{3} R d_{0} \cos \theta} \text { for } 1 \leq q \leq 6 \\
d_{q}=\sqrt{(3 R)^{2}+d_{0}^{2}+6 R d_{0} \cos \theta} \text { for } 7 \leq q \leq 12 \\
d_{q}=\sqrt{(2 \sqrt{3} R)^{2}+d_{0}^{2}+4 \sqrt{3} R d_{0} \cos \theta} \text { for } 13 \leq q \leq 18 .
\end{gathered}
$$

Substituting (B7)-(B10) to (B6), and using $\gamma=4, \sigma=8 \mathrm{~dB}$ for the integration, we obtain the numerical results as presented in Table 5.1. 